\title{
REVIEW OF THE SYNTHESIS OF LAYERED DOUBLE HYDROXIDES: A THERMODYNAMIC APPROACH
}

\section{Juan J. Bravo-Suárez}

Centro de Investigaciones en Catálisis, Escuela de Ingeniería Química, Universidad Industrial de Santander, A.A. 678 Bucaramanga, Colombia and Environmental Catalysis and Nanomaterials Laboratory, Department of Chemical Engineering, Virginia Polytechnic Institute and State University, Blacksburg, Virginia 24061-0211

\section{Edgar A. Páez-Mozo}

Centro de Investigaciones en Catálisis, Escuela de Química, Universidad Industrial de Santander, A.A. 678 Bucaramanga, Colombia S. Ted Oyama*

Environmental Catalysis and Nanomaterials Laboratory, Department of Chemical Engineering, Virginia Polytechnic Institute and State University, Blacksburg, Virginia 24061-0211

Recebido em 29/4/03; aceito em 3/11/03; publicado na web em 27/05/04

\begin{abstract}
The synthesis of layered double hydroxides (LDHs) by hydrothermal-LDH reconstruction and coprecipitation methods is reviewed using a thermodynamic approach. A mixture model was used for the estimation of the thermodynamics of formation of LDHs. The synthesis and solubility of LDHs are discussed in terms of standard molar Gibbs free energy change of reaction. Data for numerous divalent and trivalent metals as well as for some monovalent and tetravalent metals that may be part of the LDH structure have been compiled. Good agreement is found between theoretical and experimental data. Diagrams and tables for the prediction of possible new LDH materials are provided.
\end{abstract}

Keywords: layered double hydroxides; synthesis; thermodynamics.

\section{INTRODUCTION}

Layered double hydroxides (LDHs) are a broad class of inorganic lamellar compounds of basic character with high capacity for anion intercalation. The LDHs are also widely known as hydrotalcite-like compounds due to their structural similarities to hydrotalcite, a mineral with the formula $\mathrm{Mg}_{6} \mathrm{Al}_{2}(\mathrm{OH})_{16} \mathrm{CO}_{3} \cdot 4 \mathrm{H}_{2} \mathrm{O}$. The hydrotalcite structure results from the stacking of brucite-like layers $\left[\mathrm{Mg}(\mathrm{OH})_{2}\right]$ containing a positive residual charge arising from the partial isomorphous substitution of $\mathrm{Mg}^{2+}$ cations by $\mathrm{Al}^{3+}$ cations. This positive excess charge is balanced by the carbonate anions, which reside in the interlamellar spaces ${ }^{1-2}$. Natural hydrotalcite was first reported by Hochstetter ${ }^{3}$ in 1842 and synthesized 100 years later by Feitknecht ${ }^{4}$. In the last 20 years there have been numerous publications related with the synthesis and applications of $\mathrm{LDH}$ compounds $^{5-14}$. They are represented by the general formula $\left[\mathrm{M}^{2+}{ }_{1-\mathrm{x}} \mathrm{M}^{3+}{ }_{\mathrm{x}}(\mathrm{OH})_{2}\right]^{\mathrm{x+}}\left(\mathrm{A}^{\mathrm{n}-}{ }_{\mathrm{x} / \mathrm{n}}\right) \cdot \mathrm{mH}_{2} \mathrm{O}^{2}$, where $\mathrm{M}^{2+}$ and $\mathrm{M}^{3+}$ can be any divalent and trivalent metal ions (whose ionic radius is not too different from that of $\mathrm{Mg}^{2+}$ ), which can be accommodated in the octahedral holes in the brucite-like layers and $\mathrm{x}$ is the metal ratio $\mathrm{M}^{3+} /\left(\mathrm{M}^{2+}+\mathrm{M}^{3+}\right)$. The species $\mathrm{A}^{\mathrm{n}-}$ in the interlamellar region can be any charge compensating anion (organic or inorganic) and $\mathrm{m}$ is the amount of water present in the same region ${ }^{6,9}$. A special case occurs when $\mathrm{A}^{\mathrm{n}}$ - is the hydroxide ion, $\mathrm{OH}^{-}$. This class of compounds are called Meixnerite-like compounds (MLCs) because of their similarities to $\mathrm{Mg}_{6}{ }_{6} \mathrm{Al}^{3+}{ }_{2}(\mathrm{OH})_{16}(\mathrm{OH})_{2} \cdot 4 \mathrm{H}_{2} \mathrm{O}$, a compound named after Meixner ${ }^{15}$.

LDH compounds have been synthesized by direct methods, which include coprecipitation ${ }^{4,6,9}$, sol-gel synthesis ${ }^{16}$, chimie douce ${ }^{17}$, saltoxide reaction ${ }^{10}$, hydrothermal growth ${ }^{18-21}$ and electrochemical synthesis $^{22}$. Indirect methods include all syntheses that use an LDH

*e-mail: oyama@vt.edu

\# Current address: Research Institute for Innovation in Sustainable Chemistry, National Institute of Advanced Industrial Science and Technology (AIST), 16-1 Onogawa, Tsukuba 305-8569, Japan. as a precursor. Examples of these are all anion exchange based methods such as direct anion exchange ${ }^{10,23}$, anion exchange by acid attack with elimination of the guest species in the interlayer region ${ }^{24,25}$ and anion exchange by surfactant salt formation ${ }^{26}$. The non-anion exchange methods include the delamination-restacking method ${ }^{27}$ and LDH reconstruction method ${ }^{28-34}$. Metals that have been reported as constituents of LDH layers as well as some claimed in patents, ${ }^{95-52}$ are shown in Figure 1.

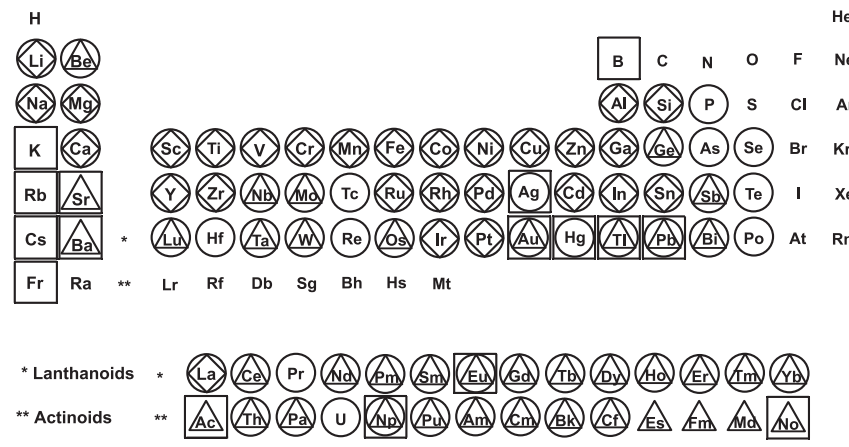

Figure 1. Metals in LDH layers, ${ }^{95-52} . \diamond$ Reported in journals, $\triangle$ Claimed in patents, $\square$ Deviation from Shannon ionic radius ${ }^{53}$ of $\mathrm{Mg}^{2+}>50 \%$, $\bigcirc$ Deviation from Shannon ionic radius of $\mathrm{Mg}^{2+}<50 \%$. Some elements have ionic radius deviations from $\mathrm{Mg}^{2+}>$ and $<50 \%$ because they may exist in different oxidation states

There is currently an increasing interest in LDHs, due to their properties as catalysts and catalyst supports ${ }^{9,12,54}$, antacids ${ }^{9,55-56}$, trapping agents for anionic contaminants ${ }^{57-60}$, flame retardants ${ }^{9,61}$, polymer stabilizers ${ }^{9,62}$, molecular sieves ${ }^{5}$, ion exchangers and adsorbents ${ }^{63,64}$. New areas have been investigated for applications in the field of medicine ${ }^{65,66}$, thin films ${ }^{67}$, conducting materials ${ }^{68,69}$, electrodes ${ }^{70-72}$ and corrosion protection ${ }^{73-75}$. LDHs have also been recently related to the origin of life ${ }^{76,77}$. 
One of the advantages of LDHs among layered materials is the great number of possible compositions and metal-anion combinations that can be synthesized. In spite of this uniqueness, there has not been a practical guide to assist in synthesizing LDH materials with specific properties.

For this reason a thermodynamic study of the reactions involved in the synthesis of LDHs is desirable. However, this is no easy task, due to the great amount of thermodynamic data required including standard free energies, enthalpies and entropies of formation for all the wide range of compositional variations of LDHs. Moreover, very few articles are found in the literature ${ }^{78-80}$ dealing with all the necessary data to make this work feasible. We have recently proposed ${ }^{81}$ and evaluated five different models for the estimation of thermodynamic properties of LDH compounds. The main idea in these models was to treat the LDH as a mixture of structurally simple compounds. In the present work, for the sake of usefulness, we use the most simple and practical model for the estimation of standard molar Gibbs free energies of formation for $\left[\mathrm{M}^{2+}{ }_{1-\mathrm{x}} \mathrm{M}^{3+}{ }_{\mathrm{x}}(\mathrm{OH})_{2}\right]^{\mathrm{x+}}\left(\mathrm{A}^{\mathrm{n}-}{ }_{\mathrm{x} / \mathrm{n}}\right)$ compounds. These free energies of formation are used for the calculation of the standard free energy changes of reaction, the basis of comparison among the different reactions under consideration. We particularly concentrate our analysis on the synthesis of LDHs by hydrothermalLDH reconstruction and coprecipitation methods. Similarly, the solubility of LDHs is also studied. An understanding of this property is important for LDH applications in aqueous media such as in the controlled release of interlayer anionic compounds by LDH layer dissolution ${ }^{65,66,82,83}$ and geochemistry.

\section{SOURCES OF THERMODYNAMIC DATA}

Thermodynamic data for a great number of compounds (oxides, hydroxides, salts and ions) are readily available in several existing compilations of thermochemical data ${ }^{84-89}$. In the cases where standard molar entropies of formation of compounds are not available, they are estimated by the method of Latimer ${ }^{90}$ as described by Naumov et $a l .{ }^{84}$. Reference states used in the thermodynamic databases are 298.15 K and $101325 \mathrm{~Pa}$ for compounds and a hypothetical ideal $1 \mathrm{~mol} \mathrm{~kg}^{-1}$ solution at $298.15 \mathrm{~K}$ and $101325 \mathrm{~Pa}$ for dissolved species.

The models we proposed for the estimation of the thermodynamic properties of formation of LDH materials based on the combinations of simple compounds in the LDH structure are:

Model 0: a combination of the compounds $\mathrm{M}^{2+}(\mathrm{OH})_{2}, \mathrm{M}^{3+}(\mathrm{OH})_{3}$ and the ion $\left(\mathrm{A}^{\mathrm{n}-}\right)$

Model 1: a combination of the compounds $\mathrm{M}^{2+}(\mathrm{OH})_{2}, \mathrm{M}^{3+}(\mathrm{OH})_{3}$ and $\mathrm{H}_{\mathrm{n}}\left(\mathrm{A}^{\mathrm{n}-}\right)$

Model 2: a combination of the compounds $\mathrm{M}^{2+}(\mathrm{OH})_{2}, \mathrm{M}^{3+}(\mathrm{OH})_{3}$ and $\mathrm{M}^{2+}\left(\mathrm{A}^{\mathrm{n}-}\right)_{2 / \mathrm{n}}$

Model 3: a combination of the compounds $\mathrm{M}^{2+}(\mathrm{OH})_{2}, \mathrm{M}^{3+}(\mathrm{OH})_{3}$ and $\mathrm{M}_{\mathrm{n}}^{3+}\left(\mathrm{A}^{\mathrm{n}-}\right)_{3}$

General Model: a combination of all the single models.

All the mixture models require thermodynamic data of the single species involved. Among all of the models, model 2 and the general model gave the best results for the estimation of the thermodynamic properties of formation. Although the general model has the attractiveness of being less arbitrary and averaging more information when available, model 2 has the advantage of simplicity, requiring less thermodynamic information than the general model. Model 2 requires thermodynamic data of metal hydroxides and salts of the divalent metal and is based on the following reaction:

$$
\begin{aligned}
(1-3 \mathrm{x} / 2) \mathrm{M}^{2+}(\mathrm{OH})_{2}+(\mathrm{x}) \mathrm{M}^{3+}(\mathrm{OH})_{3}+(\mathrm{x} / 2) \mathrm{M}^{2+}\left(\mathrm{A}^{\mathrm{n}-}\right)_{2 / \mathrm{n}}= \\
{\left[\mathrm{M}^{2+}{ }_{1-\mathrm{x}} \mathrm{M}^{3+}{ }_{\mathrm{x}}(\mathrm{OH})_{2}\right] \mathrm{A}^{\mathrm{n}-}{ }_{\mathrm{x} / \mathrm{n}} }
\end{aligned}
$$

For $\mathrm{n}=1$ or 2

The standard molar Gibbs free energy of formation at any temperature, T, of the LDHs is given by the sum of the standard molar Gibbs free energies of formation of the components:

$$
\begin{aligned}
& \Delta_{\mathrm{f}} \mathrm{G}_{\mathrm{m}}^{\mathrm{o}}\{\mathrm{T}, \mathrm{LDH}\}=(1-3 \mathrm{x} / 2) \Delta_{\mathrm{f}} \mathrm{G}_{\mathrm{m}}^{\mathrm{o}}\left\{\mathrm{T}^{\mathrm{M}} \mathrm{M}^{2+}(\mathrm{OH})_{2}\right\}+ \\
& (\mathrm{x}) \Delta_{\mathrm{f}} \mathrm{G}_{\mathrm{m}}^{\mathrm{o}}\left\{\mathrm{T}, \mathrm{M}^{3+}(\mathrm{OH})_{3}\right\}+(\mathrm{x} / 2) \Delta_{\mathrm{f}} \mathrm{G}_{\mathrm{m}}^{\mathrm{o}}\left\{\mathrm{T}, \mathrm{M}^{2+}\left(\mathrm{A}^{\mathrm{n}-}\right)_{2 / \mathrm{n}}\right\}
\end{aligned}
$$

\section{SYNTHESIS OF LDHS BY HYDROTHERMAL-LDH RECONSTRUCTION (H-R) METHODS}

Hydrothermal and LDH reconstruction methods are similar since both methods involve reactions of metal oxides, customarily in aqueous solution, with the anion of interest. A general reaction is given by:

$$
\begin{array}{r}
(1-\mathrm{x}) \mathrm{M}^{2+} \mathrm{O}+(\mathrm{x} / 2) \mathrm{M}_{2}^{3+} \mathrm{O}_{3}+(\mathrm{x} / \mathrm{n}) \mathrm{A}^{\mathrm{n}-}+(1+\mathrm{x} / 2) \mathrm{H}_{2} \mathrm{O}= \\
{\left[\mathrm{M}^{2+}{ }_{1-\mathrm{x}} \mathrm{M}^{3+}{ }_{\mathrm{x}}(\mathrm{OH})_{2}\right] \mathrm{A}^{\mathrm{n}-}{ }_{\mathrm{x} / \mathrm{n}}+(\mathrm{x}) \mathrm{OH}^{-}}
\end{array}
$$

The standard molar Gibbs free energy change of the hydrothermal-reconstruction reaction $\left(\Delta_{\mathrm{HR}} \mathrm{G}_{\mathrm{m}}^{\mathrm{o}}\right)$ is given by:

$$
\begin{aligned}
& \Delta_{\mathrm{HR}} \mathrm{G}_{\mathrm{m}}^{\mathrm{o}}\{\mathrm{T}\}= {\left[\Delta_{\mathrm{f}} \mathrm{G}_{\mathrm{m}}^{\mathrm{o}}\{\mathrm{T}, \mathrm{LDH}\}+(\mathrm{x}) \Delta_{\mathrm{f}} \mathrm{G}_{\mathrm{m}}^{\mathrm{o}}\left\{\mathrm{T}, \mathrm{OH}^{-}\right\}\right]-} \\
& {\left[(1-\mathrm{x}) \Delta_{\mathrm{f}} \mathrm{G}_{\mathrm{m}}^{\mathrm{o}}\left\{\mathrm{T}, \mathrm{M}^{2+} \mathrm{O}\right\}+(\mathrm{x} / 2) \Delta_{\mathrm{f}} \mathrm{G}_{\mathrm{m}}^{\mathrm{o}}\left\{\mathrm{T}, \mathrm{M}_{2}{ }^{3+} \mathrm{O}_{3}\right\}+\right.} \\
&\left.(\mathrm{x} / 2) \Delta_{\mathrm{f}} \mathrm{G}_{\mathrm{m}}^{\mathrm{o}}\left\{\mathrm{T}, \mathrm{A}^{\mathrm{n}-}\right\}+(1+\mathrm{x} / 2) \Delta_{\mathrm{f}} \mathrm{G}_{\mathrm{m}}^{\mathrm{o}}\left\{\mathrm{T}, \mathrm{H}_{2} \mathrm{O}\right\}\right]
\end{aligned}
$$

Two cases can be distinguished: 1) Hydroxyl anion $\left(\mathrm{A}^{\mathrm{n}-}=\mathrm{OH}^{-}\right)$ and 2) Non-hydroxyl anions $\left(\mathrm{A}^{\mathrm{n}-} \neq \mathrm{OH}^{-}\right)$.

\section{Synthesis of hydroxyl anion based LDHs by H-R methods: meixnerite-like compounds (MLCs)}

MLC refers to layered double hydroxides in which the interlamellar anion is $\mathrm{OH}^{-}$. Replacing $\mathrm{OH}^{-}$in reactions 1 and 3:

$$
\begin{gathered}
(1-\mathrm{x}) \mathrm{M}^{2+}(\mathrm{OH})_{2}+(\mathrm{x}) \mathrm{M}^{3+}(\mathrm{OH})_{3}=\left[\mathrm{M}^{2+}{ }_{1-\mathrm{x}} \mathrm{M}^{3+}{ }_{\mathrm{x}}(\mathrm{OH})_{2}\right] \mathrm{OH}_{\mathrm{x}} \\
(1-\mathrm{x}) \mathrm{M}^{2+} \mathrm{O}+(\mathrm{x} / 2) \mathrm{M}_{2}^{3+} \mathrm{O}_{3}+(1+\mathrm{x} / 2) \mathrm{H}_{2} \mathrm{O}= \\
{\left[\mathrm{M}^{2+}{ }_{1-\mathrm{x}} \mathrm{M}^{3+}{ }_{\mathrm{x}}(\mathrm{OH})_{2}\right] \mathrm{OH}_{\mathrm{x}}}
\end{gathered}
$$

For the thermodynamic calculations, reactions 5 and 6 are combined:

$$
\begin{aligned}
&(1-\mathrm{x}) \mathrm{M}^{2+} \mathrm{O}+(\mathrm{x} / 2) \mathrm{M}_{2}^{3+} \mathrm{O}_{3}+(1+\mathrm{x} / 2) \mathrm{H}_{2} \mathrm{O}= \\
&(1-\mathrm{x}) \mathrm{M}^{2+}(\mathrm{OH})_{2}+(\mathrm{x}) \mathrm{M}^{3+}(\mathrm{OH})_{3}
\end{aligned}
$$

Terms in the lhs of Equation 7 are rearranged in the following way:

$$
\begin{aligned}
&(1-\mathrm{x})\left[\mathrm{M}^{2+} \mathrm{O}+\mathrm{H}_{2} \mathrm{O}\right]+(\mathrm{x})[\left.(1 / 2) \mathrm{M}_{2}^{3+} \mathrm{O}_{3}+(3 / 2) \mathrm{H}_{2} \mathrm{O}\right]= \\
&(1-\mathrm{x}) \mathrm{M}^{2+}(\mathrm{OH})_{2}+(\mathrm{x}) \mathrm{M}^{3+}(\mathrm{OH})_{3}
\end{aligned}
$$

Using $\Delta_{R} G_{m}^{o}\{T\}=\sum_{i}^{p} \Delta_{f} G_{m}^{o}\{T$, Products $i\}-\sum_{j}^{r} \Delta_{f} G_{m}^{o}\{T$, Reac tan ts j\}, then

$$
\begin{gathered}
\Delta_{\mathrm{HR}} \mathrm{G}_{\mathrm{m}}^{\mathrm{o}}\{\mathrm{T}, \mathrm{MLC}\}=(1-\mathrm{x}) \Delta_{\mathrm{f}} \mathrm{G}_{\mathrm{m}}^{\mathrm{o}}\left\{\mathrm{T}, \mathrm{M}^{2+}(\mathrm{OH})_{2}\right\}-\Delta_{\mathrm{f}} \mathrm{G}_{\mathrm{m}}^{\mathrm{o}}\left\{\mathrm{T}, \mathrm{M}^{2+} \mathrm{O}\right\}- \\
\left.\Delta_{\mathrm{f}} \mathrm{G}_{\mathrm{m}}^{\mathrm{o}}\left\{\mathrm{T}, \mathrm{H}_{2} \mathrm{O}\right\}\right]+(\mathrm{x})\left[\Delta_{\mathrm{f}} \mathrm{G}_{\mathrm{m}}^{\mathrm{o}}\left\{\mathrm{T}, \mathrm{M}^{3+}(\mathrm{OH})_{3}\right\}-\right. \\
\left.(1 / 2) \Delta_{\mathrm{f}} \mathrm{G}_{\mathrm{m}}^{\mathrm{o}}\left\{\mathrm{T}, \mathrm{M}_{2}^{3+} \mathrm{O}_{3}\right\}-(3 / 2) \Delta_{\mathrm{f}} \mathrm{G}_{\mathrm{m}}^{\mathrm{o}}\left\{\mathrm{T}, \mathrm{H}_{2} \mathrm{O}\right\}\right]
\end{gathered}
$$

Equation 9 is a linear combination of the hydration reactions of the divalent and trivalent oxides:

$\mathrm{M}^{2+} \mathrm{O}+\mathrm{H}_{2} \mathrm{O}=\mathrm{M}^{2+}(\mathrm{OH})_{2}$ 


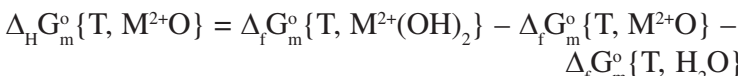

$(1 / 2) \mathrm{M}_{2}^{3+} \mathrm{O}_{3}+(3 / 2) \mathrm{H}_{2} \mathrm{O}=\mathrm{M}^{2+}(\mathrm{OH})_{2}$

$\Delta_{\mathrm{H}} \mathrm{G}_{\mathrm{m}}^{\mathrm{o}}\left\{\mathrm{T}, \mathrm{M}_{2}^{3+} \mathrm{O}_{3}\right\}=\Delta_{\mathrm{f}} \mathrm{G}_{\mathrm{m}}^{\mathrm{o}}\left\{\mathrm{T}, \mathrm{M}^{3+}(\mathrm{OH})_{3}\right\}-(1 / 2) \Delta_{\mathrm{f}} \mathrm{G}_{\mathrm{m}}^{\circ}\left\{\mathrm{T}, \mathrm{M}_{2}^{3+} \mathrm{O}_{3}\right\}-$

$$
(3 / 2) \Delta_{\mathrm{f}} \mathrm{G}_{\mathrm{m}}^{\mathrm{o}}\left\{\mathrm{T}, \mathrm{H}_{2} \mathrm{O}\right\}
$$

Equation 9 is rewritten in terms of Gibbs free energies of hydration of oxides:

$$
\Delta_{\mathrm{HR}} \mathrm{G}_{\mathrm{m}}^{\mathrm{o}}\{\mathrm{T}, \mathrm{MLC}\}=(1-\mathrm{x}) \Delta_{\mathrm{H}} \mathrm{G}_{\mathrm{m}}^{\mathrm{o}}\left\{\mathrm{T}, \mathrm{M}^{2+} \mathrm{O}\right\}+(\mathrm{x}) \Delta_{\mathrm{H}} \mathrm{G}_{\mathrm{m}}^{\mathrm{o}}\left\{\mathrm{T}, \mathrm{M}_{2}^{3+} \mathrm{O}_{3}\right\}
$$

This result is not surprising if we account for the assumptions in the mixture model, as seen in Equations 5 and 7 and stated by Allada et al. ${ }^{78}$ : "Metal and anion coordination environments in the hydrotalcite are structurally similar, and therefore probably energetically similar, to those in the simple minerals used as components".

\section{Gibbs free energies of hydration for metal oxides at different temperatures}

Data for the standard molar Gibbs free energy change of hydration at different temperatures are given in Figures 2 and 3. Results for the hydration reactions of some $\mathrm{M}^{+}$and $\mathrm{M}^{2+}$ oxides can be seen in Figure 2 . Some hydration reactions of $\mathrm{M}^{3+}$ and $\mathrm{M}^{4+}$ oxides are also shown in Figure 3. All the calculations are based on the following wellknown thermodynamic relationships ${ }^{84-85}$ :

$\Delta_{\mathrm{f}} \mathrm{G}_{\mathrm{m}}^{\mathrm{o}}\{\mathrm{T}\}=\Delta_{\mathrm{f}} \mathrm{H}_{\mathrm{m}}^{\mathrm{o}}\{\mathrm{T}\}-\mathrm{T} \cdot \mathrm{S}_{\mathrm{m}}^{\mathrm{o}}\{\mathrm{T}\}$

$\Delta_{\mathrm{f}} \mathrm{H}_{\mathrm{m}}^{\mathrm{o}}\{\mathrm{T}\}=\Delta_{\mathrm{f}} \mathrm{H}_{\mathrm{m}}^{\mathrm{o}}\{298.15 \mathrm{~K}\}+\int_{298.15 \mathrm{~K}}^{\mathrm{T}} \mathrm{C}_{\mathrm{p}, \mathrm{m}}^{\mathrm{o}} \mathrm{dT}$

$\mathrm{S}_{\mathrm{m}}^{\mathrm{o}}\{\mathrm{T}\}=\mathrm{S}_{\mathrm{m}}^{\mathrm{o}}\{298.15 \mathrm{~K}\}+\int_{298.15 \mathrm{~K}}^{\mathrm{T}} \mathrm{C}_{\mathrm{p}, \mathrm{m}}^{\mathrm{o}} \mathrm{dT} / \mathrm{T}$

Where $\mathrm{C}_{\mathrm{p}, \mathrm{m}}^{\mathrm{o}}$ is a function of temperature of the following form:

$\mathrm{C}_{\mathrm{p}, \mathrm{m}}^{\mathrm{o}}=\mathrm{a}+\mathrm{b} \cdot 10^{-3} \mathrm{~T}+\mathrm{c} \cdot 10^{6} \mathrm{~T}^{-2}+\mathrm{d} \cdot 10^{-6} \mathrm{~T}^{2}$

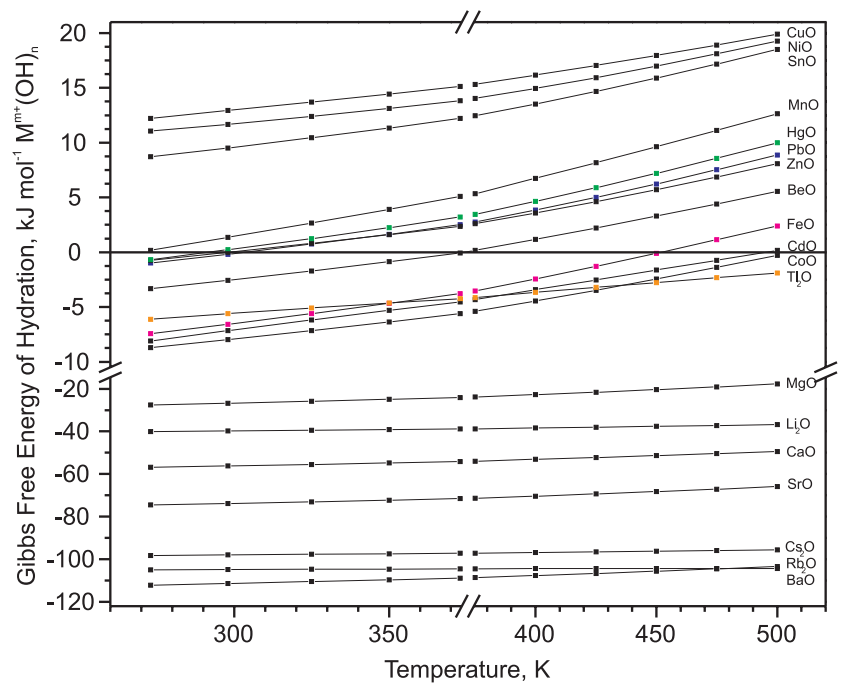

Figure 2. Standard molar Gibbs free energy change of hydration for some $\mathrm{M}^{+}$and $\mathrm{M}^{2+}$ oxides at different temperatures

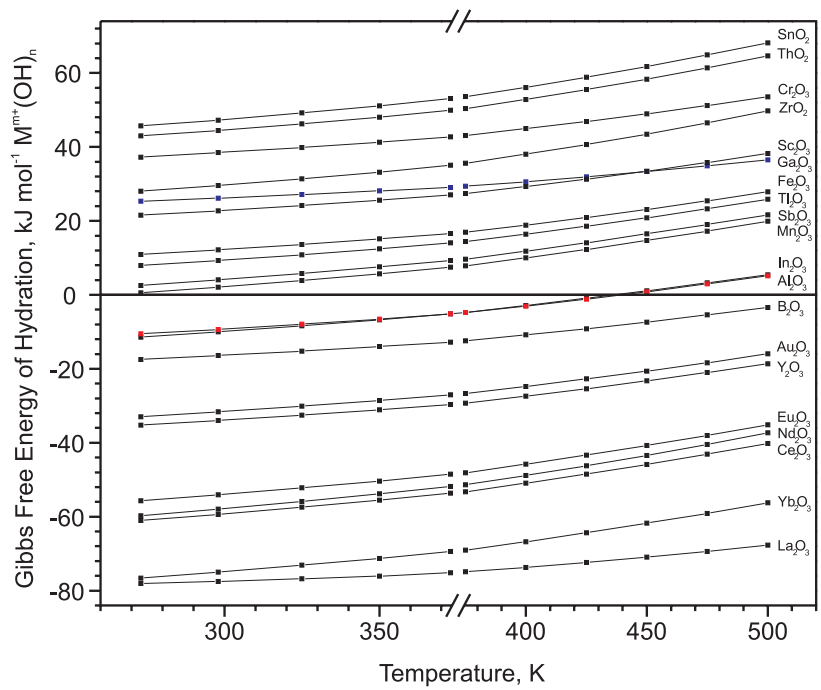

Figure 3. Standard molar Gibbs free energy change of hydration for some $\mathrm{M}^{3+}$ and $\mathrm{M}^{4+}$ oxides at different temperatures

Combining Equation 18 with Equations 16 and 17 and substituting the obtained results in Equation 15, the following expressions are obtained:

$$
\begin{aligned}
& \Delta_{\mathrm{f}} \mathrm{G}_{\mathrm{m}}^{\mathrm{o}}\{\mathrm{T}\}=\mathrm{a}[\{\mathrm{T}-298.15\}-\mathrm{T} \cdot \ln (\mathrm{T} / 298.15)]+ \\
& \mathrm{b}\left[0.5 \cdot 10^{-3}\left(\mathrm{~T}^{2}-298.15^{2}\right)-\mathrm{T} \cdot 10^{-3}(\mathrm{~T}-298.15)\right]+ \\
& \mathrm{c}\left[10^{6}\left(298.15^{-1}-\mathrm{T}^{-1}\right)+\mathrm{T} \cdot 10^{6}\left(\mathrm{~T}^{-2}-298.15^{-2}\right) / 2\right]+ \\
& \mathrm{d}\left[10^{-6}\left(\mathrm{~T}^{3}-298.15^{3}\right) / 3-\mathrm{T} \cdot 10^{-6}\left(\mathrm{~T}^{2}-298.15^{2}\right) / 2\right]+ \\
& \Delta_{\mathrm{f}} \mathrm{H}_{\mathrm{m}}^{\mathrm{o}}\{298.15\}-\mathrm{T} \cdot \Delta_{\mathrm{f}} \mathrm{S}_{\mathrm{m}}^{\mathrm{o}}\{298.15 \mathrm{~K}\} \\
& \Delta_{\mathrm{f}} \mathrm{G}_{\mathrm{m}}^{\mathrm{o}}\{\mathrm{T}\}=\mathrm{a}[(\mathrm{T}-298.15)-\mathrm{T} \cdot \ln (\mathrm{T} / 298.15)]+ \\
& \mathrm{b}\left[0.5 \cdot 10^{-3}\left(\mathrm{~T}^{2}-298.15^{2}\right)-\mathrm{T} \cdot 10^{-3}(\mathrm{~T}-298.15)\right]+ \\
& \mathrm{c}\left[10^{6}\left(298.15^{-1}-\mathrm{T}^{-1}\right)+\mathrm{T} \cdot 10^{6}\left(\mathrm{~T}^{-2}-298.15^{-2}\right) / 2\right]+ \\
& \mathrm{d}\left[10^{-6}\left(\mathrm{~T}^{3}-298.15^{3}\right) / 3-\mathrm{T} \cdot 10^{-6}\left(\mathrm{~T}^{2}-298.15^{2}\right) / 2\right]+ \\
& \Delta_{\mathrm{f}} \mathrm{G}_{\mathrm{m}}^{\mathrm{o}}\{298.15 \mathrm{~K}\}-(\mathrm{T}-298.15) \cdot \mathrm{S}_{\mathrm{m}}^{\mathrm{o}}\{298.15 \mathrm{~K}\}
\end{aligned}
$$

The selection of Equations 19 or 20 for $\Delta_{\mathrm{f}} \mathrm{G}_{\mathrm{m}}^{\mathrm{o}}\{\mathrm{T}\}$ calculations is mainly based on the availability of the thermodynamic properties of the compounds. Equations 19 or 20 are applied to each compound in Equations 10 and 12. At a specific temperature the standard molar Gibbs free energy of hydration is calculated by the expression:

$\Delta_{\mathrm{H}} \mathrm{G}_{\mathrm{m}}^{\mathrm{o}}\{\mathrm{T}\}=\sum_{\mathrm{i}}^{\mathrm{p}} \Delta_{\mathrm{f}} \mathrm{G}_{\mathrm{m}}^{\mathrm{o}}\{\mathrm{T}$, Products $\mathrm{i}\}-\sum_{\mathrm{j}}^{\mathrm{r}} \Delta_{\mathrm{f}} \mathrm{G}_{\mathrm{m}}^{\mathrm{o}}\{\mathrm{T}$, Reac tan ts $\mathrm{j}\}$

For temperatures over $373 \mathrm{~K}$, a constant pressure of $5 \mathrm{MPa}$ is used. In general, for solids and liquids there is little noticeable change of $\Delta_{\mathrm{f}} \mathrm{G}_{\mathrm{m}}^{\mathrm{o}}\{\mathrm{T}\}$ with pressure due to the relatively small changes in molar volumes ${ }^{91,92}$. Data of $\mathrm{C}_{\mathrm{p}, \mathrm{m}}^{\mathrm{o}}$ as a function of temperature for compressed water at a pressure of $5 \mathrm{MPa}$ were taken from Perry et $a l^{89}$.

The standard molar Gibbs free energy change of hydration for many $\mathrm{M}^{+}, \mathrm{M}^{2+}, \mathrm{M}^{3+}$ and $\mathrm{M}^{4+}$ oxides are given in Tables 1 and 2. Information about the reactions, equations used, calculated $\Delta_{\mathrm{H}} \mathrm{G}_{\mathrm{m}}^{\mathrm{o}}\{\mathrm{T}\}$ 's and references for each of the entries are also provided in Tables 1 and 2. When Equations 19 and 20 are listed for one of the reactions, it indicates that either equation can be applied since there are enough published thermodynamic data.

Data in Tables 1 and 2 together with Equation 14 are used to estimate the standard molar Gibbs free energy change of reaction for the synthesis of MLCs by H-R methods. These results are summarized schematically in Figure 4 as a nomogram, and some typical examples 
Table 1. Standard molar Gibbs free energy change of the hydration reaction for some $\mathrm{M}^{+}$and $\mathrm{M}^{2+}$ oxides at $298.15 \mathrm{~K}$

\begin{tabular}{|c|c|c|c|}
\hline Hydration Reaction & Equations & $\Delta_{\mathrm{H}} \mathrm{G}_{\mathrm{m}}^{\mathrm{o}}\left\{298.15 \mathrm{~K}, \mathrm{M}^{\mathrm{m}+}(\mathrm{OH})_{\mathrm{n}}\right\}, \mathrm{kJ} \mathrm{mol}^{-1}$ & References \\
\hline $1 / 2 \mathrm{Ag}_{2} \mathrm{O}(\mathrm{s})+1 / 2 \mathrm{H}_{2} \mathrm{O}(\mathrm{l})=\mathrm{AgOH}(\mathrm{s})$ & 20 & -1.6 & 87,89 \\
\hline $1 / 2 \mathrm{Tl}_{2} \mathrm{O}(\mathrm{s})+1 / 2 \mathrm{H}_{2} \mathrm{O}(\mathrm{l})=\mathrm{TlOH}(\mathrm{s})$ & 19 & -5.6 & $86,88,89,92$ \\
\hline $1 / 2 \mathrm{Li}_{2}^{2} \mathrm{O}(\mathrm{s})+1 / 2 \mathrm{H}_{2}^{2} \mathrm{O}(\mathrm{l})=\mathrm{LiOH}(\mathrm{s})$ & 19 & -39.8 & $86,88,89$ \\
\hline $1 / 2 \mathrm{Cs}_{2}^{2} \mathrm{O}(\mathrm{s})+1 / 2 \mathrm{H}_{2} \mathrm{O}(\mathrm{l})=\mathrm{CsOH}(\mathrm{s})$ & 19 & -97.9 & $86,88,89$ \\
\hline $1 / 2 \mathrm{Na}_{2}^{2} \mathrm{O}(\mathrm{s})+1 / 2 \mathrm{H}_{2}^{2} \mathrm{O}(\mathrm{l})=\mathrm{NaOH}(\mathrm{s})$ & $19-20$ & -74.1 & $86,87,89$ \\
\hline $1 / 2 \mathrm{Rb}_{2}^{2} \mathrm{O}(\mathrm{s})+1 / 2 \mathrm{H}_{2}^{2} \mathrm{O}(\mathrm{l})=\mathrm{RbOH}(\mathrm{s})$ & 19 & -104.8 & $86,88,89$ \\
\hline $\mathrm{PdO}(\mathrm{s})+\mathrm{H}_{2} \mathrm{O}(\mathrm{l})=\mathrm{Pd}(\mathrm{OH})_{2}(\mathrm{~s})$ & 19 & 144.8 & $86-87,89$ \\
\hline $\mathrm{TiO}(\mathrm{s})+\mathrm{H}_{2} \mathrm{O}(\mathrm{l})=\mathrm{Ti}(\mathrm{OH})_{2}(\mathrm{~s})$ & 19 & 33.5 & $86,87,89,90$ \\
\hline $\mathrm{CuO}(\mathrm{s})+\mathrm{H}_{2}^{2} \mathrm{O}(\mathrm{l})=\mathrm{Cu}(\mathrm{OH})_{2}(\mathrm{~s})$ & 20 & 12.9 & $85,86,89,90$ \\
\hline $\mathrm{NiO}(\mathrm{s})+\mathrm{H}_{2}^{2} \mathrm{O}(\mathrm{l})=\mathrm{Ni}(\mathrm{OH})_{2}(\mathrm{~s})$ & 20 & 11.7 & $86,89,90,92$ \\
\hline $\mathrm{SnO}(\mathrm{s})+\mathrm{H}_{2} \mathrm{O}(\mathrm{l})=\mathrm{Sn}(\mathrm{OH})_{2}(\mathrm{~s})$ & $19-20$ & 9.5 & $86,89,90,92$ \\
\hline $\mathrm{MnO}(\mathrm{s})+\mathrm{H}_{2} \mathrm{O}(\mathrm{l})=\mathrm{Mn}(\mathrm{OH})_{2}(\mathrm{~s})$ & $19-20$ & 1.3 & $86,89,90,92$ \\
\hline $\mathrm{HgO}(\mathrm{s})+\mathrm{H}_{2} \mathrm{O}(\mathrm{l})=\mathrm{Hg}(\mathrm{OH})_{2}(\mathrm{~s})$ & $19-20$ & 0.2 & $86,89,90,92$ \\
\hline $\mathrm{ZnO}(\mathrm{s})+\mathrm{H}_{2}^{2} \mathrm{O}(\mathrm{l})=\mathrm{Zn}(\mathrm{OH})_{2}(\mathrm{~s})$ & $19-20$ & -0.1 & $86,89,90,92$ \\
\hline $\mathrm{PbO}(\mathrm{s})+\mathrm{H}_{2} \mathrm{O}(\mathrm{l})=\mathrm{Pb}(\mathrm{OH})_{2}(\mathrm{~s})$ & $19-20$ & -0.2 & $86,89,90,92$ \\
\hline $\mathrm{PtO}(\mathrm{s})+\mathrm{H}_{2} \mathrm{O}(\mathrm{l})=\mathrm{Pt}(\mathrm{OH})_{2}(\mathrm{~s})$ & 20 & -0.9 & 87,89 \\
\hline $\mathrm{BeO}(\mathrm{s})+\mathrm{H}_{2} \mathrm{O}(\mathrm{l})=\mathrm{Be}(\mathrm{OH})_{2}(\mathrm{~s})$ & $19-20$ & -2.6 & $86,88,89$ \\
\hline $\mathrm{FeO}(\mathrm{s})+\mathrm{H}_{2}^{2} \mathrm{O}(\mathrm{l})=\mathrm{Fe}(\mathrm{OH})_{2}(\mathrm{~s})$ & $19-20$ & -6.6 & $86,88,89$ \\
\hline $\mathrm{CdO}(\mathrm{s})+\mathrm{H}_{2} \mathrm{O}(\mathrm{l})=\mathrm{Cd}(\mathrm{OH})_{2}(\mathrm{~s})$ & $19-20$ & -7.2 & $85,86,89$ \\
\hline $\mathrm{CoO}(\mathrm{s})+\mathrm{H}_{2}^{2} \mathrm{O}(\mathrm{l})=\mathrm{Co}(\mathrm{OH})_{2}(\mathrm{~s})$ & $19-20$ & -8.0 & $86,88,89$ \\
\hline $\mathrm{MgO}(\mathrm{s})+\mathrm{H}_{2} \mathrm{O}(\mathrm{l})=\mathrm{Mg}(\mathrm{OH})_{2}(\mathrm{~s})$ & $19-20$ & -26.8 & 86,89 \\
\hline $\mathrm{CaO}(\mathrm{s})+\mathrm{H}_{2} \mathrm{O}(\mathrm{l})=\mathrm{Ca}(\mathrm{OH})_{2}(\mathrm{~s})$ & $19-20$ & -56.2 & 86,89 \\
\hline $\mathrm{SrO}(\mathrm{s})+\mathrm{H}_{2} \mathrm{O}(\mathrm{l})=\mathrm{Sr}(\mathrm{OH})_{2}(\mathrm{~s})$ & $19-20$ & -73.9 & $85,86,89$ \\
\hline $\mathrm{RaO}(\mathrm{s})+\mathrm{H}_{2} \mathrm{O}(\mathrm{l})=\mathrm{Ra}(\mathrm{OH})_{2}(\mathrm{~s})$ & 19 & -110.1 & $86,87,89,90$ \\
\hline $\mathrm{BaO}(\mathrm{s})+\mathrm{H}_{2}^{2} \mathrm{O}(\mathrm{l})=\mathrm{Ba}(\mathrm{OH})_{2}(\mathrm{~s})$ & $19-20$ & -111.4 & $86,88,89$ \\
\hline
\end{tabular}

Table 2. Standard molar Gibbs free energy change of the hydration reaction for some $\mathrm{M}^{3+}$ and $\mathrm{M}^{4+}$ oxides at $298.15 \mathrm{~K}$

\begin{tabular}{|c|c|c|c|}
\hline Hydration Reaction & Equations & $\Delta_{\mathrm{H}} \mathrm{G}_{\mathrm{m}}^{\mathrm{o}}\left\{298.15 \mathrm{~K}, \mathrm{M}^{\mathrm{m}+}(\mathrm{OH})_{\mathrm{n}}\right\}, \mathrm{kJ} \mathrm{mol}^{-1}$ & References \\
\hline $1 / 2 \mathrm{Pu}_{2} \mathrm{O}_{3}(\mathrm{~s})+3 / 2 \mathrm{H}_{2} \mathrm{O}(\mathrm{l})=\mathrm{Pu}(\mathrm{OH})_{3}(\mathrm{~s})$ & 20 & 105.9 & 87,89 \\
\hline $1 / 2 \mathrm{Tm}_{2}^{2} \mathrm{O}_{3}(\mathrm{~s})+3 / 2 \mathrm{H}_{2}^{2} \mathrm{O}(\mathrm{l})=\mathrm{Tm}(\mathrm{OH})_{3}(\mathrm{~s})$ & 20 & 55.7 & $86,87,89$ \\
\hline $1 / 2 \mathrm{Cr}_{2} \mathrm{O}_{3}(\mathrm{~s})+3 / 2 \mathrm{H}_{2} \mathrm{O}(\mathrm{l})=\mathrm{Cr}(\mathrm{OH})_{3}(\mathrm{~s})$ & 20 & 38.5 & $86,89,92$ \\
\hline $1 / 2 \mathrm{Bi}_{2} \mathrm{O}_{3}(\mathrm{~s})+3 / 2 \mathrm{H}_{2} \mathrm{O}(\mathrm{l})=\mathrm{Bi}(\mathrm{OH})_{3}(\mathrm{~s})$ & $19-20$ & 27.6 & $86,87,89$ \\
\hline $1 / 2 \mathrm{Ga}_{2} \mathrm{O}_{3}(\mathrm{~s})+3 / 2 \mathrm{H}_{2}^{2} \mathrm{O}(\mathrm{l})=\mathrm{Ga}(\mathrm{OH})_{3}(\mathrm{~s})$ & $19-20$ & 26.2 & $86,89,92$ \\
\hline $1 / 2 \mathrm{Sc}_{2} \mathrm{O}_{3}(\mathrm{~s})+3 / 2 \mathrm{H}_{2} \mathrm{O}(\mathrm{l})=\mathrm{Sc}(\mathrm{OH})_{3}(\mathrm{~s})$ & $19-20$ & 22.7 & $86,87,89,92$ \\
\hline $1 / 2-\mathrm{Ti}_{2} \mathrm{O}_{3}(\mathrm{~s})+3 / 2 \mathrm{H}_{2} \mathrm{O}(\mathrm{l})=\mathrm{Ti}(\mathrm{OH})_{3}(\mathrm{~s})$ & 19 & 15.9 & $86,87,89,90$ \\
\hline $1 / 2 \mathrm{Fe}_{2} \mathrm{O}_{3}(\mathrm{~s})+3 / 2 \mathrm{H}_{2} \mathrm{O}(\mathrm{l})=\mathrm{Fe}(\mathrm{OH})_{3}(\mathrm{~s})$ & $19-20$ & 12.1 & $86,89,92$ \\
\hline $1 / 2 \mathrm{Tl}_{2} \mathrm{O}_{3}(\mathrm{~s})+3 / 2 \mathrm{H}_{2} \mathrm{O}(\mathrm{l})=\mathrm{Tl}(\mathrm{OH})_{3}(\mathrm{~s})$ & $19-20$ & 9.8 & $86,89,92$ \\
\hline $1 / 2 \mathrm{~V}_{2} \mathrm{O}_{3}(\mathrm{~s})+3 / 2 \mathrm{H}_{2}^{2} \mathrm{O}(\mathrm{l})=\mathrm{V}(\mathrm{OH})_{3}(\mathrm{~s})$ & 19 & 7.0 & $86,87,89,90$ \\
\hline $1 / 2 \mathrm{Sb}_{2}^{2} \mathrm{O}_{3}(\mathrm{~s})+3 / 2 \mathrm{H}_{2} \mathrm{O}(\mathrm{l})=\mathrm{Sb}(\mathrm{OH})_{3}(\mathrm{~s})$ & $19-20$ & 4.0 & $86,89,92$ \\
\hline $1 / 2 \mathrm{Mn}_{2} \mathrm{O}_{3}(\mathrm{~s})+3 / 2 \mathrm{H}_{2} \mathrm{O}(\mathrm{l})=\mathrm{Mn}(\mathrm{OH})_{3}(\mathrm{~s})$ & $19-20$ & 2.1 & $86,89,90,92$ \\
\hline $1 / 2 \mathrm{Lu}_{2} \mathrm{O}_{3}(\mathrm{~s})+3 / 2 \mathrm{H}_{2} \mathrm{O}(\mathrm{l})=\mathrm{Lu}(\mathrm{OH})_{3}(\mathrm{~s})$ & $19-20$ & -8.6 & $86,87,89,90$ \\
\hline $1 / 2 \mathrm{In}_{2} \mathrm{O}_{3}(\mathrm{~s})+3 / 2 \mathrm{H}_{2} \mathrm{O}(\mathrm{l})=\mathrm{In}(\mathrm{OH})_{3}(\mathrm{~s})$ & $19-20$ & -9.4 & $86,89,92$ \\
\hline $1 / 2 \mathrm{Al}_{2} \mathrm{O}_{3}(\mathrm{~s})+3 / 2 \mathrm{H}_{2} \mathrm{O}(\mathrm{l})=\mathrm{Al}(\mathrm{OH})_{3}(\mathrm{~s})$ & $19-20$ & -10.0 & 86,89 \\
\hline $1 / 2 \mathrm{~B}_{2} \mathrm{O}_{3}(\mathrm{~s})+3 / 2 \mathrm{H}_{2} \mathrm{O}(\mathrm{l})=\mathrm{B}(\mathrm{OH})_{3}(\mathrm{~s})$ & $19-20$ & -16.4 & $86,89,92$ \\
\hline $1 / 2 \mathrm{Er}_{2} \mathrm{O}_{3}(\mathrm{~s})+3 / 2 \mathrm{H}_{2} \mathrm{O}(\mathrm{l})=\mathrm{Er}(\mathrm{OH})_{3}(\mathrm{~s})$ & 20 & -30.0 & 87,89 \\
\hline $1 / 2 \mathrm{Au}_{2} \mathrm{O}_{3}(\mathrm{~s})+3 / 2 \mathrm{H}_{2} \mathrm{O}(\mathrm{l})=\mathrm{Au}(\mathrm{OH})_{3}(\mathrm{~s})$ & $19-20$ & -31.7 & $85,86,88,89$ \\
\hline $1 / 2 \mathrm{Y}_{2} \mathrm{O}_{3}(\mathrm{~s})+3 / 2 \mathrm{H}_{2} \mathrm{O}(\mathrm{l})=\mathrm{Y}(\mathrm{OH})_{3}(\mathrm{~s})$ & $19-20$ & -34.0 & $86,89,92$ \\
\hline $1 / 2 \mathrm{Dy}_{2} \mathrm{O}_{3}(\mathrm{~s})+3 / 2 \mathrm{H}_{2} \mathrm{O}(\mathrm{l})=\mathrm{Dy}(\mathrm{OH})_{3}(\mathrm{~s})$ & 20 & -51.5 & $86,87,89$ \\
\hline $1 / 2 \mathrm{Eu}_{2} \mathrm{O}_{3}(\mathrm{~s})+3 / 2 \mathrm{H}_{2}^{2} \mathrm{O}(\mathrm{l})=\mathrm{Eu}(\mathrm{OH})_{3}(\mathrm{~s})$ & $19-20$ & -54.1 & $86,89,92$ \\
\hline $1 / 2 \mathrm{Pr}_{2} \mathrm{O}_{3}(\mathrm{~s})+3 / 2 \mathrm{H}_{2} \mathrm{O}(\mathrm{l})=\operatorname{Pr}(\mathrm{OH})_{3}(\mathrm{~s})$ & 20 & -54.9 & 87,89 \\
\hline $1 / 2 \mathrm{Nd}_{2} \mathrm{O}_{3}(\mathrm{~s})+3 / 2 \mathrm{H}_{2} \mathrm{O}(\mathrm{l})=\mathrm{Nd}(\mathrm{OH})_{3}(\mathrm{~s})$ & $19-20$ & -57.9 & $86,89,92$ \\
\hline $1 / 2 \mathrm{Ce}_{2} \mathrm{O}_{3}^{3}(\mathrm{~s})+3 / 2 \mathrm{H}_{2}^{2} \mathrm{O}(\mathrm{l})=\mathrm{Ce}(\mathrm{OH})_{3}(\mathrm{~s})$ & $19-20$ & -59.4 & $86,89,92$ \\
\hline $1 / 2 \mathrm{~Tb}_{2} \mathrm{O}_{3}(\mathrm{~s})+3 / 2 \mathrm{H}_{2} \mathrm{O}(\mathrm{l})=\mathrm{Tb}(\mathrm{OH})_{3}(\mathrm{~s})$ & 20 & -71.2 & $86,87,89$ \\
\hline $1 / 2 \mathrm{Yb}_{2} \mathrm{O}_{3}(\mathrm{~s})+3 / 2 \mathrm{H}_{2} \mathrm{O}(\mathrm{l})=\mathrm{Yb}(\mathrm{OH})_{3}(\mathrm{~s})$ & 20 & -75.0 & $86,89,92$ \\
\hline $1 / 2 \mathrm{La}_{2} \mathrm{O}_{3}(\mathrm{~s})+3 / 2 \mathrm{H}_{2} \mathrm{O}(\mathrm{l})=\mathrm{La}(\mathrm{OH})_{3}(\mathrm{~s})$ & $19-20$ & -77.5 & $86,89,92$ \\
\hline $1 / 2 \mathrm{Gd}_{2} \mathrm{O}_{3}(\mathrm{~s})+3 / 2 \mathrm{H}_{2} \mathrm{O}(\mathrm{l})=\mathrm{Gd}(\mathrm{OH})_{3}(\mathrm{~s})$ & 20 & -93.4 & 87,89 \\
\hline $1 / 2 \mathrm{Sm}_{2} \mathrm{O}_{3}(\mathrm{~s})+3 / 2 \mathrm{H}_{2} \mathrm{O}(\mathrm{l})=\mathrm{Sm}(\mathrm{OH})_{3}(\mathrm{~s})$ & 20 & -94.4 & $86,87,89$ \\
\hline 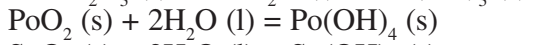 & 20 & 125.4 & 87,89 \\
\hline $\mathrm{SnO}_{2}^{2}(\mathrm{~s})+2 \mathrm{H}_{2}^{2} \mathrm{O}(\mathrm{l})=\mathrm{Sn}(\mathrm{OH})_{4}(\mathrm{~s})$ & $19-20$ & 47.3 & $86,89,92$ \\
\hline $\mathrm{ThO}_{2}(\mathrm{~s})+2 \mathrm{H}_{2} \mathrm{O}(\mathrm{l})=\mathrm{Th}(\mathrm{OH})_{4}(\mathrm{~s})$ & $19-20$ & 44.4 & $86,89,92$ \\
\hline $\mathrm{UO}_{2}(\mathrm{~s})+2 \mathrm{H}_{2} \mathrm{O}(\mathrm{l})=\mathrm{U}(\mathrm{OH})_{4}(\mathrm{~s})$ & 20 & 36.3 & 87,89 \\
\hline $\mathrm{ZrO}_{2}(\mathrm{~s})+2 \mathrm{H}_{2} \mathrm{O}(\mathrm{l})=\mathrm{Zr}(\mathrm{OH})_{4}(\mathrm{~s})$ & $19-20$ & 29.5 & $86,89,92$ \\
\hline $\mathrm{AmO}_{2}^{2}(\mathrm{~s})+2 \mathrm{H}_{2}^{2} \mathrm{O}(\mathrm{l})=\mathrm{Am}(\mathrm{OH})_{4}(\mathrm{~s})$ & 20 & -25.6 & 87,89 \\
\hline
\end{tabular}




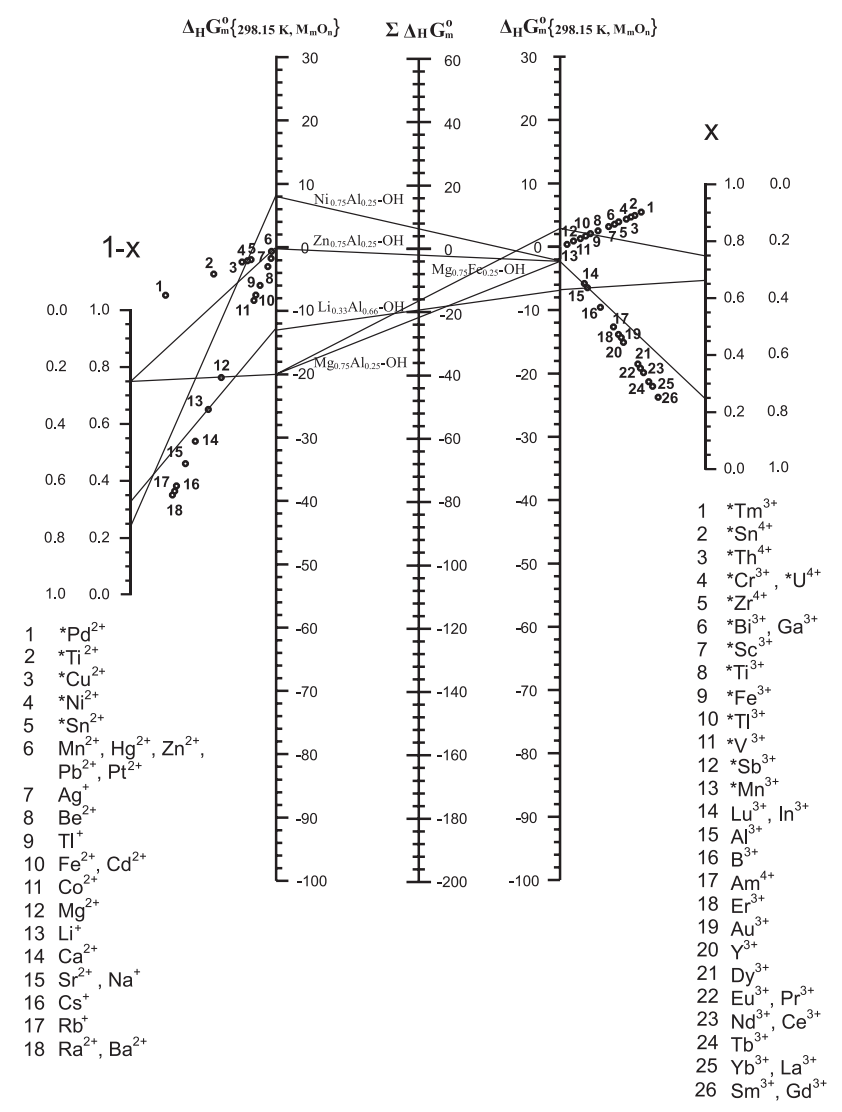

Figure 4. Estimated standard molar Gibbs free energy change of reaction for the synthesis of MLCs by H-R methods at $298.15 \mathrm{~K}$, in $\mathrm{kJ} \mathrm{mol}^{-1}$

of MLCs are shown. To estimate the standard free energy change of reaction at a temperature different from $298.15 \mathrm{~K}$, data from Figures 2 and 3 can be used.

The $\Delta_{\mathrm{HR}} \mathrm{G}_{\mathrm{m}}^{\mathrm{o}}\{\mathrm{T}\}$ for a MLC is easily estimated taking into account its formula. For a MLC with the general formula: $\left[\mathrm{M}^{2+}{ }_{1-\mathrm{x}} \mathrm{M}^{3+}{ }_{\mathrm{x}}(\mathrm{OH})_{2}\right]^{\mathrm{x+}}$ $(\mathrm{OH})_{\mathrm{x}}$, the $\Delta_{\mathrm{HR}} \mathrm{G}_{\mathrm{m}}^{\mathrm{o}}\{\mathrm{T}\}$ is the result of hydration contributions from $\mathrm{M}^{2+}$ and $\mathrm{M}^{3+}$ oxides. Given a metal ratio, $\mathrm{x}$, the contribution from the hydration of $\mathrm{M}^{3+}$ oxides is obtained by drawing a straight line from the $\mathrm{x}$ scale, going through the selected $\mathrm{M}^{3+}$ mark and finally intersecting the $\Delta_{\mathrm{H}} \mathrm{G}_{\mathrm{m}}^{\mathrm{o}}\{298.15 \mathrm{~K}\}$ right axis. Similarly, the contribution from the hydration of $\mathrm{M}^{2+}$ oxides is obtained, but now using the 1-x values and the scale on the left of Figure 4. Finally, the intersect formed from the straight line, joining the resulting contributions from the $\mathrm{M}^{2+}$ and $\mathrm{M}^{3+}$ oxides, and the central axis gives the estimated standard molar Gibbs free energy change of reaction for the synthesis of MLCs by H-R methods. When an asterisk precedes one of the $\mathrm{M}^{3+}$ and $\mathrm{M}^{2+}$ metals, then $\mathrm{x}$ and 1-x inverted scales should be used, respectively. Although the previous procedure is intended for $\mathrm{M}^{2+}-\mathrm{M}^{3+}$ metal mixed oxides, it is not restricted to that specific case. For instance, $\mathrm{M}^{+}-\mathrm{M}^{3+}$ metal mixed oxides among others can also be easily analyzed by using a proper general formula.

\section{Synthesis of non-hydroxyl anion based LDH by H-R methods}

For thermodynamic calculations, Equations 1 and 3 are combined and rearranged to give:

$$
\begin{aligned}
&(1-\mathrm{x}) {\left[\mathrm{M}^{2+} \mathrm{O}+\mathrm{H}_{2} \mathrm{O}\right]+\left[(\mathrm{x} / 2) \mathrm{M}_{2}^{3+} \mathrm{O}_{3}+(3 \mathrm{x} / 2) \mathrm{H}_{2} \mathrm{O}\right]+(\mathrm{x} / \mathrm{n}) \mathrm{A}^{\mathrm{n}-} } \\
&+(\mathrm{x} / 2) \mathrm{M}^{2+}(\mathrm{OH})_{2}=(1-\mathrm{x})\left[\mathrm{M}^{2+}(\mathrm{OH})_{2}\right]+(\mathrm{x})\left[\mathrm{M}^{3+}(\mathrm{OH})_{3}\right] \\
&+(\mathrm{x} / 2) \mathrm{M}^{2+}\left(\mathrm{A}^{\mathrm{n}-}\right)_{2 / \mathrm{n}}+(\mathrm{x}) \mathrm{OH}^{-}
\end{aligned}
$$

Similarly to Equation 9, the standard Gibbs free energy change of the reaction is:

$$
\begin{aligned}
& \Delta_{\mathrm{HR}} \mathrm{G}_{\mathrm{m}}^{\mathrm{o}}\{\mathrm{T}, \mathrm{LDH}-\mathrm{A}\}=(1-\mathrm{x}) \Delta_{\mathrm{H}} \mathrm{G}_{\mathrm{m}}^{\mathrm{o}}\left\{\mathrm{T}, \mathrm{M}^{2+} \mathrm{O}\right\}+(\mathrm{x}) \Delta_{\mathrm{H}} \mathrm{G}_{\mathrm{m}}^{\mathrm{o}}\left\{\mathrm{T}, \mathrm{M}_{2}^{3+} \mathrm{O}_{3}\right\} \\
& \quad+(\mathrm{x})\left[(1 / 2) \Delta_{\mathrm{f}} \mathrm{G}_{\mathrm{m}}^{\mathrm{o}}\left\{\mathrm{T}, \mathrm{M}^{2+}\left(\mathrm{A}^{\mathrm{n}-}\right)_{2 / \mathrm{n}}\right\}-(1 / 2) \Delta_{\mathrm{f}} \mathrm{G}_{\mathrm{m}}^{\mathrm{o}}\left\{\mathrm{T}, \mathrm{M}^{2+}(\mathrm{OH})_{2}\right\}\right. \\
& \left.+\Delta_{\mathrm{f}} \mathrm{G}_{\mathrm{m}}^{\mathrm{o}}\left\{\mathrm{T}, \mathrm{OH}^{-}\right\}-(1 / \mathrm{n}) \Delta_{\mathrm{f}} \mathrm{G}_{\mathrm{m}}^{\mathrm{o}}\left\{\mathrm{T}, \mathrm{A}^{\mathrm{n}-}\right\}\right]
\end{aligned}
$$

The term in brackets may be regarded as a contribution due to anion difference in the MLCs $\left(\Delta_{\mathrm{C}} \mathrm{G}_{\mathrm{m}}^{\mathrm{o}}\left\{\mathrm{T}, \mathrm{M}^{2+} \mathrm{A}\right\}\right)$.

$$
\begin{aligned}
\Delta_{\mathrm{C}} \mathrm{G}_{\mathrm{m}}^{\mathrm{o}} & \left\{\mathrm{T}, \mathrm{M}^{2+} \mathrm{A}\right\}=(1 / 2) \Delta_{\mathrm{f}} \mathrm{G}_{\mathrm{m}}^{\mathrm{o}}\left\{\mathrm{T}, \mathrm{M}^{2+}\left(\mathrm{A}^{\mathrm{n}-}\right)_{2 / \mathrm{n}}\right\}-(1 / 2) \Delta_{\mathrm{f}} \mathrm{G}_{\mathrm{m}}^{\mathrm{o}}\left\{\mathrm{T}^{\mathrm{N}} \mathrm{M}^{2+}(\mathrm{OH})_{2}\right\} \\
& +\Delta_{\mathrm{f}} \mathrm{G}_{\mathrm{m}}^{\mathrm{o}}\left\{\mathrm{T}, \mathrm{OH}^{-}\right\}-(1 / \mathrm{n}) \Delta_{\mathrm{f}} \mathrm{G}_{\mathrm{m}}^{\mathrm{o}}\left\{\mathrm{T}, \mathrm{A}^{\mathrm{n}-}\right\}
\end{aligned}
$$

Finally,

$$
\begin{aligned}
& \Delta_{\mathrm{HR}} \mathrm{G}_{\mathrm{m}}^{\mathrm{o}}\{\mathrm{T}, \mathrm{LDH}-\mathrm{A}\}=(1-\mathrm{x}) \Delta_{\mathrm{H}} \mathrm{G}_{\mathrm{m}}^{\mathrm{o}}\left\{\mathrm{T}, \mathrm{M}^{2+} \mathrm{O}\right\}+(\mathrm{x}) \Delta_{\mathrm{H}} \mathrm{G}_{\mathrm{m}}^{\mathrm{o}}\left\{\mathrm{T}, \mathrm{M}_{2}^{3+} \mathrm{O}_{3}\right\} \\
& \quad+(\mathrm{x}) \Delta_{\mathrm{C}} \mathrm{G}_{\mathrm{m}}^{\mathrm{o}}\left\{\mathrm{T}, \mathrm{M}^{2+} \mathrm{A}\right\}
\end{aligned}
$$

Equation 24 is used for the calculation of $\Delta_{C} G_{m}^{o}\left\{T, M^{2+} A\right\}$. For example, the standard Gibbs free energy for the anion contribution term in the $\left[\mathrm{Mg}^{2+}{ }_{1-x} \mathrm{M}^{3+}{ }_{\mathrm{x}}(\mathrm{OH})_{2}\right] \mathrm{Cl}_{\mathrm{x}} \mathrm{LDH}$ is calculated by:

$$
\begin{aligned}
& \Delta_{\mathrm{C}} \mathrm{G}_{\mathrm{m}}^{\mathrm{o}}\{\left.298.15 \mathrm{~K}, \mathrm{Mg}^{2+} \mathrm{A}\right\}=(1 / 2) \Delta_{\mathrm{f}} \mathrm{G}_{\mathrm{m}}^{\mathrm{o}}\left\{298.15 \mathrm{~K}, \mathrm{Mg}(\mathrm{Cl})_{2}\right\}- \\
&(1 / 2) \Delta_{\mathrm{f}} \mathrm{G}_{\mathrm{m}}^{\mathrm{o}}\left\{298.15 \mathrm{~K}, \mathrm{Mg}(\mathrm{OH})_{2}\right\}+\Delta_{\mathrm{f}} \mathrm{G}_{\mathrm{m}}^{\mathrm{o}}\left\{298.15 \mathrm{~K}, \mathrm{OH}^{-}\right\}- \\
& \Delta_{\mathrm{f}} \mathrm{G}_{\mathrm{m}}^{\mathrm{o}}\left\{298.15 \mathrm{~K}, \mathrm{Cl}^{-}\right\} \\
& \Delta_{\mathrm{C}} \mathrm{G}_{\mathrm{m}}^{\mathrm{o}}\left\{298.15 \mathrm{~K}, \mathrm{Mg}^{2+} \mathrm{A}\right\}=(1 / 2)(-591.8)-(1 / 2)(-833.6)+(-157.3) \\
& \quad-(-131.3)=94.9 \mathrm{~kJ} \mathrm{~mol}^{-1}
\end{aligned}
$$

Other results shown in Table 3 are calculated in a similar manner. The thermodynamic data of the compounds were taken from Naumov et al. ${ }^{86}$, Karapet'yants et al. ${ }^{87}$ and Perry et al. ${ }^{89}$. The results of Figure 4 combined with values in Table 3 can be substituted into Equation 25 to estimate standard Gibbs free energies of the synthesis of LDHs by $\mathrm{H}-\mathrm{R}$ methods. For instance, the standard Gibbs free energy for the synthesis of a $\left[\mathrm{Mg}^{2+}{ }_{0.75} \mathrm{Al}^{3+}{ }_{0.25}(\mathrm{OH})_{2}\right] \mathrm{F}_{0.25} \mathrm{LDH}$ by H-R methods is obtained from:

$$
\begin{aligned}
\Delta_{\mathrm{HR}} \mathrm{G}_{\mathrm{m}}^{\mathrm{o}}\{298.15 \mathrm{~K}, \mathrm{MgAl}-\mathrm{F}\}=0.75 \Delta_{\mathrm{H}} \mathrm{G}_{\mathrm{m}}^{\mathrm{o}}\{298.15 \mathrm{~K}, \mathrm{MgO}\}+ \\
0.25 \Delta_{\mathrm{H}} \mathrm{G}_{\mathrm{m}}^{\mathrm{o}}\left\{298.15 \mathrm{~K}, \mathrm{Al}_{2} \mathrm{O}_{3}\right\}+0.25 \Delta_{\mathrm{C}} \mathrm{G}_{\mathrm{m}}^{\mathrm{o}}\{298.15 \mathrm{~K}, \mathrm{MgF}\}
\end{aligned}
$$

Table 3. Standard molar Gibbs free energy anion contribution term, $\Delta_{\mathrm{C}} \mathrm{G}_{\mathrm{m}}^{\mathrm{o}}\left\{298.15 \mathrm{~K}, \mathrm{M}^{2+} \mathrm{A}\right\}$

\begin{tabular}{lrrrrrrrr}
\hline & \multicolumn{7}{c}{$\Delta_{\mathrm{C}} \mathrm{G}_{\mathrm{m}}^{\mathrm{o}}\left\{298.15 \mathrm{~K}, \mathrm{M}^{2+} \mathrm{A}\right\}, \mathrm{kJ} \mathrm{mol}^{-1}$} \\
\cline { 3 - 9 } $\mathrm{M}^{2+}$ & \multicolumn{1}{c}{$\mathrm{I}^{-}$} & \multicolumn{1}{c}{$\mathrm{Br}^{-}$} & \multicolumn{1}{c}{$\mathrm{Cl}^{-}$} & \multicolumn{1}{c}{$\mathrm{NO}_{3}^{-}$} & $\mathrm{SO}_{4}^{2-}$ & $\mathrm{CO}_{3}^{2-}$ & \multicolumn{1}{c}{$\mathrm{F}^{-}$} & $\mathrm{OH}^{-}$ \\
\hline $\mathrm{Ba}^{2+}$ & 22.3 & 9.5 & 2.7 & -15.8 & -31.5 & -33.7 & -20.8 & 0 \\
$\mathrm{Be}^{2+}$ & 197.3 & 179.0 & 160.4 & 0.0 & 76.8 & 41.8 & 48.7 & 0 \\
$\mathrm{Ca}^{2+}$ & 75.9 & 34.6 & 46.3 & 31.6 & 15.0 & -9.2 & -16.8 & 0 \\
$\mathrm{Cd}^{2+}$ & 29.6 & 36.3 & 39.1 & 59.4 & 40.6 & 9.4 & 34.4 & 0 \\
$\mathrm{Co}^{2+}$ & 73.4 & 69.5 & 64.8 & 66.7 & 50.6 & 10.4 & 44.0 & 0 \\
$\mathrm{Cu}^{2+}$ & 61.2 & 73.6 & 66.9 & 71.9 & 62.3 & 26.5 & 58.9 & 0 \\
$\mathrm{Fe}^{2+}$ & 71.7 & 71.6 & 64.1 & 43.2 & 47.7 & 11.7 & 50.9 & 0 \\
$\mathrm{Hg}^{2+}$ & -8.2 & 13.5 & 32.3 & 69.1 & 67.3 & 20.0 & 89.5 & 0 \\
$\mathrm{Mg}^{2+}$ & 131.2 & 114.1 & 94.9 & 76.7 & 49.4 & 17.8 & 3.9 & 0 \\
$\mathrm{Mn}^{2+}$ & 61.6 & 63.3 & 53.1 & 5.4 & 36.1 & -2.3 & 46.8 & 0 \\
$\mathrm{Ni}^{2+}$ & 70.7 & 61.6 & 65.3 & 57.1 & 44.0 & 21.5 & 36.7 & 0 \\
$\mathrm{~Pb}^{2+}$ & 21.3 & 30.9 & 30.8 & 41.8 & 22.8 & 6.7 & 26.7 & 0 \\
$\mathrm{Pd}^{2+}$ & -5.1 & 45.9 & 44.3 & -68.0 & -34.3 & -45.4 & 20.9 & 0 \\
$\mathrm{Sn}^{2+}$ & 68.1 & 68.1 & 89.9 & -51.8 & -18.1 & -12.4 & 33.4 & 0 \\
$\mathrm{Sr}^{2+}$ & 48.3 & 27.8 & 13.5 & -5.9 & -18.6 & -27.3 & -22.5 & 0 \\
$\mathrm{Zn}^{2+}$ & 67.1 & 67.9 & 65.4 & 81.9 & 57.6 & 16.9 & 43.2 & 0 \\
\hline
\end{tabular}


From Figure 4:

$0.75 \Delta_{\mathrm{H}} \mathrm{G}_{\mathrm{m}}^{\mathrm{o}}\{298.15 \mathrm{~K}, \mathrm{MgO}\}+0.25 \Delta_{\mathrm{H}} \mathrm{G}_{\mathrm{m}}^{\mathrm{o}}\left\{298.15 \mathrm{~K}, \mathrm{Al}_{2} \mathrm{O}_{3}\right\}$

$\approx-22.5 \mathrm{~kJ} \mathrm{~mol}^{-1}$

And from Table 3:

$0.25 \Delta_{\mathrm{C}} \mathrm{G}_{\mathrm{m}}^{\mathrm{o}}\{298.15 \mathrm{~K}, \mathrm{MgF}\} \approx 1.0 \mathrm{~kJ} \mathrm{~mol}^{-1}$

Thus

$\Delta_{\mathrm{HR}} \mathrm{G}_{\mathrm{m}}^{\mathrm{o}}\{298.15 \mathrm{~K}, \mathrm{MgAl}-\mathrm{F}\} \approx-21.5 \mathrm{~kJ} \mathrm{~mol}^{-1}$

\section{SYNTHESIS OF LDHS BY THE COPRECIPITATION METHOD}

In the coprecipitacion method, LDHs are generally precipitated from a mixture of metal salts and the interlamellar anion of interest under basic conditions. As previously stated, the different combinations of these metal salts and anions are numerous. In this work, a number of these combinations are studied.

\section{Case 1: divalent and trivalent metal with the same univalent anion}

$$
\begin{gathered}
(1-\mathrm{x}) \mathrm{M}^{2+}\left(\mathrm{B}^{-}\right)_{2}+(\mathrm{x}) \mathrm{M}^{3+}\left(\mathrm{B}^{-}\right)_{3}+2 \mathrm{OH}^{-}+(\mathrm{x} / \mathrm{n}) \mathrm{A}^{\mathrm{n}-}= \\
{\left[\mathrm{M}^{2+}{ }_{1-\mathrm{x}} \mathrm{M}^{3+}{ }_{\mathrm{x}}(\mathrm{OH})_{2}\right] \mathrm{A}^{\mathrm{n}-}{ }_{\mathrm{x} / \mathrm{n}}+(2+\mathrm{x}) \mathrm{B}^{-}}
\end{gathered}
$$

Replacing Equation 1 in Equation 26, the standard Gibbs free energy change of reaction for coprecipitation $(C P),\left(\Delta_{C P} G_{m}^{o}\{T, L D H\}\right)$, can be expressed by:

$$
\begin{aligned}
\Delta_{\mathrm{CP}} \mathrm{G}_{\mathrm{m}}^{\mathrm{o}} & \{\mathrm{T}, \mathrm{LDH}\}=(1-\mathrm{x})\left[\Delta_{\mathrm{f}} \mathrm{G}_{\mathrm{m}}^{\mathrm{o}}\left\{\mathrm{T}, \mathrm{M}^{2+}(\mathrm{OH})_{2}\right\}-\Delta_{\mathrm{f}} \mathrm{G}_{\mathrm{m}}^{\mathrm{o}}\left\{\mathrm{T}, \mathrm{M}^{2+}\left(\mathrm{B}^{-}\right)_{2}\right\}\right] \\
& +(\mathrm{x})\left[\Delta_{\mathrm{f}} \mathrm{G}_{\mathrm{m}}^{\mathrm{o}}\left\{\mathrm{T}, \mathrm{M}^{3+}(\mathrm{OH})_{3}\right\}-\Delta_{\mathrm{f}} \mathrm{G}_{\mathrm{m}}^{\mathrm{o}}\left\{\mathrm{T}, \mathrm{M}^{3+}\left(\mathrm{B}^{-}\right)_{3}\right\}\right]+ \\
& (\mathrm{x})\left[(1 / 2) \Delta_{\mathrm{f}} \mathrm{G}_{\mathrm{m}}^{\mathrm{o}}\left\{\mathrm{T}, \mathrm{M}^{2+}\left(\mathrm{A}^{\mathrm{n}-}\right)_{2 / \mathrm{n}}\right\}-(1 / 2) \Delta_{\mathrm{f}} \mathrm{G}_{\mathrm{m}}^{\mathrm{o}}\left\{\mathrm{T}, \mathrm{M}^{2+}(\mathrm{OH})_{2}\right\}+\right. \\
& \left.\Delta_{\mathrm{f}} \mathrm{G}_{\mathrm{m}}^{\mathrm{o}}\left\{\mathrm{T}, \mathrm{OH}^{-}\right\}-(1 / \mathrm{n})_{\mathrm{f}} \mathrm{G}_{\mathrm{m}}^{\mathrm{o}}\left\{\mathrm{T}, \mathrm{A}^{\mathrm{n}-}\right\}\right]+(2+\mathrm{x})\left[\Delta_{\mathrm{f}} \mathrm{G}_{\mathrm{m}}^{\mathrm{o}}\left\{\mathrm{T}, \mathrm{B}^{-}\right\}-\right. \\
& \left.\Delta_{\mathrm{f}} \mathrm{G}_{\mathrm{m}}^{\mathrm{o}}\left\{\mathrm{T}, \mathrm{OH}^{-}\right\}\right]
\end{aligned}
$$

The third term in Equation 27 is the free energy anion contribution term as given by Equation 24. Finally, the standard molar Gibbs free energy change of reaction is:

$$
\begin{aligned}
\Delta_{\mathrm{CP}} \mathrm{G}_{\mathrm{m}}^{\mathrm{o}} & \{\mathrm{T}, \mathrm{LDH}\}=(1-\mathrm{x})\left[\Delta_{\mathrm{f}} \mathrm{G}_{\mathrm{m}}^{\mathrm{o}}\left\{\mathrm{T}, \mathrm{M}^{2+}(\mathrm{OH})_{2}\right\}-\Delta_{\mathrm{f}} \mathrm{G}_{\mathrm{m}}^{\mathrm{o}}\left\{\mathrm{T}, \mathrm{M}^{2+}\left(\mathrm{B}^{-}\right)_{2}\right\}\right] \\
& +(\mathrm{x})\left[\Delta_{\mathrm{f}} \mathrm{G}_{\mathrm{m}}^{\mathrm{o}}\left\{\mathrm{T}, \mathrm{M}^{3+}(\mathrm{OH})_{3}\right\}-\Delta_{\mathrm{f}} \mathrm{G}_{\mathrm{m}}^{\mathrm{o}}\left\{\mathrm{T}, \mathrm{M}^{3+}\left(\mathrm{B}^{-}\right)_{3}\right\}\right]+ \\
& (\mathrm{x})\left[\Delta_{\mathrm{C}} \mathrm{G}_{\mathrm{m}}^{\mathrm{o}}\left\{\mathrm{T}, \mathrm{M}^{2+} \mathrm{A}\right\}\right]+(2)\left[\Delta_{\mathrm{f}} \mathrm{G}_{\mathrm{m}}^{\mathrm{o}}\left\{\mathrm{T}, \mathrm{B}^{-}\right\}-\Delta_{\mathrm{f}} \mathrm{G}_{\mathrm{m}}^{\mathrm{o}}\left\{\mathrm{T}, \mathrm{OH}^{-}\right\}\right]+ \\
& (\mathrm{x})\left[\Delta_{\mathrm{f}} \mathrm{G}_{\mathrm{m}}^{\mathrm{o}}\left\{\mathrm{T}, \mathrm{B}^{-}\right\}-\Delta_{\mathrm{f}} \mathrm{G}_{\mathrm{m}}^{\mathrm{o}}\left\{\mathrm{T}, \mathrm{OH}^{-}\right\}\right]
\end{aligned}
$$

From Equation 28, five different contributions (terms in brackets) to the Gibbs free energy of the synthesis of LDHs by coprecipitation are considered:

1) Contribution due to the difference in the anion of the starting divalent metal from the divalent metal hydroxide.

2) Contribution due to the difference in the anion of the starting trivalent metal from the trivalent metal hydroxide.

3) Contribution due to the anion in the final LDH.

4) Contribution due to the free anions remaining in solution from divalent metal.

5) Contribution due to the free anions remaining in solution from trivalent metal.

Calculated values for the first, second and third contribution terms of Equation 28 are given in Tables 4, 5 and 3, respectively. Results for the fourth and fifth contribution terms are given in the last row of Table 4. Several examples of the calculations are:

$\mathrm{Zn}(\mathrm{Cl})_{2}$ first contribution term to the standard Gibbs free energy of the $\mathrm{CP}$ reaction:

$$
\begin{gathered}
\Delta_{\mathrm{f}} \mathrm{G}_{\mathrm{m}}^{\mathrm{o}}\left\{298.15 \mathrm{~K}, \mathrm{Zn}(\mathrm{OH})_{2}\right\}-\Delta_{\mathrm{f}} \mathrm{G}_{\mathrm{m}}^{\mathrm{o}}\left\{298.15 \mathrm{~K}, \mathrm{Zn}(\mathrm{Cl})_{2}\right\}= \\
-554.4-(-371.5)=-182.9 \mathrm{~kJ} \mathrm{~mol}^{-1}
\end{gathered}
$$

\begin{tabular}{|c|c|c|c|c|c|c|c|c|c|}
\hline \multirow[b]{2}{*}{$\mathrm{M}^{2+}$} & \multicolumn{6}{|c|}{ Contribution Term, $\mathrm{kJ} \mathrm{mol}^{-1}, \mathrm{~B}^{-}$} & & \multicolumn{2}{|c|}{$\mathrm{D}^{2-}$, Equation 34} \\
\hline & $\mathrm{I}^{-}$ & $\mathrm{Br}^{-}$ & $\mathrm{Cl}^{-}$ & $\mathrm{F}^{-}$ & $\mathrm{NO}_{3}^{-}$ & $\mathrm{OH}^{-}$ & & $\mathrm{SO}_{4}^{2-}$ & $\mathrm{CO}_{3}^{2-}$ \\
\hline$\overline{\mathrm{Ba}^{2+}}$ & -256.1 & -125.1 & -57.4 & 287.0 & -60.1 & 0 & & 492.2 & 280.7 \\
\hline $\mathrm{Be}^{2+}$ & -605.9 & -464.1 & -372.8 & 148.0 & -91.7 & 0 & & 275.6 & 129.6 \\
\hline $\mathrm{Ca}^{2+}$ & -363.3 & -175.3 & -144.7 & 279.0 & -154.8 & 0 & & 399.2 & 231.6 \\
\hline $\mathrm{Cd}^{2+}$ & -270.7 & -178.7 & -130.2 & 176.6 & -210.5 & 0 & & 348.0 & 194.6 \\
\hline $\mathrm{Co}^{2+}$ & -358.2 & -245.2 & -181.6 & 157.3 & -225.1 & 0 & & 328.1 & 192.5 \\
\hline $\mathrm{Cu}^{2+}$ & -333.9 & -253.3 & -185.9 & 127.6 & -235.6 & 0 & & 304.6 & 160.3 \\
\hline $\mathrm{Fe}^{2+}$ & -354.8 & -249.4 & -180.3 & 143.5 & -178.2 & 0 & & 333.9 & 190.0 \\
\hline $\mathrm{Hg}^{2+}$ & -195.0 & -133.1 & -116.7 & 66.4 & -229.9 & 0 & & 294.6 & 173.2 \\
\hline $\mathrm{Mg}^{2+}$ & -473.8 & -334.4 & -241.8 & 237.5 & -245.1 & 0 & & 330.4 & 177.7 \\
\hline $\mathrm{Mn}^{2+}$ & -334.5 & -232.8 & -158.2 & 151.9 & -102.5 & 0 & & 357.1 & 218.0 \\
\hline $\mathrm{Ni}^{2+}$ & -352.7 & -229.3 & -182.6 & 172.0 & -205.9 & 0 & & 341.2 & 170.3 \\
\hline $\mathrm{Pb}^{2+}$ & -254.1 & -167.9 & -113.6 & 192.0 & -175.3 & 0 & & 383.7 & 200.0 \\
\hline $\mathrm{Pd}^{2+}$ & -201.3 & -198.0 & -140.7 & 203.6 & -74.6 & 0 & & 497.8 & 304.1 \\
\hline $\mathrm{Sn}^{2+}$ & -347.7 & -242.3 & -231.9 & 178.7 & -138.2 & 0 & & 465.4 & 238.1 \\
\hline $\mathrm{Sr}^{2+}$ & -307.9 & -161.8 & -79.1 & 290.4 & -79.9 & 0 & & 466.4 & 267.9 \\
\hline $\mathrm{Zn}^{2+}$ & -345.6 & -241.9 & -182.9 & 158.9 & -255.6 & 0 & & 314.1 & 179.4 \\
\hline$\left(\mathrm{B}^{-}-\mathrm{OH}^{-}\right)$ & 105.7 & 53.1 & 26.0 & -122.7 & 45.9 & 0 & $\left(\mathrm{D}^{2-/ 2-\mathrm{OH}^{-}}\right)$ & -214.6 & -106.7 \\
\hline
\end{tabular}

$\mathrm{Al}(\mathrm{Cl})_{3}$ second contribution term to the standard Gibbs free energy of the $\mathrm{CP}$ reaction:

$$
\begin{gathered}
\Delta_{\mathrm{f}} \mathrm{G}_{\mathrm{m}}^{\mathrm{o}}\left\{298.15 \mathrm{~K}, \mathrm{Al}(\mathrm{OH})_{3}\right\}-\Delta_{\mathrm{f}} \mathrm{G}_{\mathrm{m}}^{\mathrm{o}}\left\{298.15 \mathrm{~K}, \mathrm{Al}(\mathrm{Cl})_{3}\right\}= \\
-1156.9-(-737.7)=-419.2 \mathrm{~kJ} \mathrm{~mol}^{-1}
\end{gathered}
$$

$\mathrm{Cl}^{-}$fourth contribution term to the standard Gibbs free energy of the $\mathrm{CP}$ reaction:

$$
\begin{gathered}
\Delta_{\mathrm{f}} \mathrm{G}_{\mathrm{m}}^{\mathrm{o}}\left\{298.15 \mathrm{~K}, \mathrm{Cl}^{-}\right\}-\Delta_{\mathrm{f}} \mathrm{G}_{\mathrm{m}}^{\mathrm{o}}\left\{298.15 \mathrm{~K}, \mathrm{OH}^{-}\right\}= \\
-131.3-(-157.3)=26.0 \mathrm{~kJ} \mathrm{~mol}^{-1}
\end{gathered}
$$

The thermodynamic data of the compounds for the first and second contribution term were taken from Naumov et al. ${ }^{86}$, Karapet'yants et al..$^{87}$ and Perry et al. ${ }^{89}$. For the fourth contribution term data for the ionic species comes from Naumov et al. ${ }^{86}$.

Table 4. Standard molar Gibbs free energy contribution term of starting divalent metal cation for Equation 28 at $298.15 \mathrm{~K}^{\mathrm{a}}$

${ }^{\mathrm{a}}$ Results for the contribution term of free anions in solution are given in last row. 
Table 5. Standard molar Gibbs free energy contribution term of starting trivalent metal cation for Equation 28 at $298.15 \mathrm{~K}$

\begin{tabular}{|c|c|c|c|c|c|c|c|}
\hline \multirow[b]{2}{*}{$\mathbf{M}^{3+}$} & \multicolumn{6}{|c|}{ Contribution Term, $\mathrm{kJ} \mathrm{mol}^{-1}$, B- } & \multirow{2}{*}{$\frac{\mathrm{D}^{2-}, \text { Equation } 32}{\mathrm{SO}_{4}{ }^{2-}}$} \\
\hline & $\mathrm{I}^{-}$ & $\mathrm{Br}^{-}$ & $\mathrm{Cl}^{-}$ & $\mathrm{F}^{-}$ & $\mathrm{NO}_{3}^{-}$ & $\mathrm{OH}^{-}$ & \\
\hline $\mathrm{Al}^{3+}$ & -845.6 & -668.4 & -419.2 & 261.7 & -377.9 & 0 & 390.2 \\
\hline $\mathrm{Au}^{3+}$ & -397.6 & -324.3 & -300.4 & -51.5 & $\mathrm{x}$ & 0 & $\mathrm{x}$ \\
\hline $\mathrm{Bi}^{3+}$ & -407.5 & -283.3 & -267.8 & 338.2 & $\mathrm{x}$ & 0 & $\mathrm{x}$ \\
\hline $\mathrm{Ce}^{3+}$ & -586.2 & -319.9 & -250.6 & 481.2 & $\mathrm{x}$ & 0 & 558.9 \\
\hline $\mathrm{Cr}^{3+}$ & -644.3 & -333.5 & -257.9 & 196.2 & $\mathrm{x}$ & 0 & 704.0 \\
\hline $\mathrm{Dy}^{3+}$ & -678.6 & -398.2 & -357.7 & $\mathrm{x}$ & $\mathrm{x}$ & 0 & 753.2 \\
\hline $\mathrm{Er}^{3+}$ & -710.0 & -436.1 & -406.3 & $\mathrm{x}$ & $\mathrm{x}$ & 0 & 656.7 \\
\hline $\mathrm{Eu}^{3+}$ & -474.0 & -264.3 & -218.0 & $\mathrm{x}$ & $\mathrm{x}$ & 0 & 1158.5 \\
\hline $\mathrm{Fe}^{3+}$ & -593.7 & -468.2 & -378.2 & 296.2 & $\mathrm{x}$ & 0 & 626.1 \\
\hline $\mathrm{Ga}^{3+}$ & -618.4 & -482.4 & -343.1 & 105.4 & $\mathrm{x}$ & 0 & 691.0 \\
\hline $\mathrm{Gd}^{3+}$ & -676.6 & -384.1 & -357.7 & $\mathrm{X}$ & $\mathrm{x}$ & 0 & 517.7 \\
\hline $\mathrm{In}^{3+}$ & -638.5 & -410.0 & -317.1 & 182.0 & $\mathrm{x}$ & 0 & 502.9 \\
\hline $\mathrm{La}^{3+}$ & -591.2 & -386.6 & -254.8 & 482.0 & $\mathrm{x}$ & 0 & $\mathrm{x}$ \\
\hline $\mathrm{Lu}^{3+}$ & -711.3 & -472.8 & -380.3 & $\mathrm{x}$ & $\mathrm{x}$ & 0 & 1023.4 \\
\hline $\mathrm{Nd}^{3+}$ & -613.8 & -348.7 & -284.6 & $\mathrm{x}$ & $\mathrm{x}$ & 0 & 538.2 \\
\hline $\operatorname{Pr}^{3+}$ & -623.4 & -329.1 & -288.7 & 382.0 & $\mathrm{x}$ & 0 & 488.7 \\
\hline $\mathrm{Pu}^{3+}$ & -613.4 & -409.2 & -277.0 & 321.3 & $\mathrm{x}$ & 0 & $\mathrm{x}$ \\
\hline $\mathrm{Sb}^{3+}$ & -578.2 & -433.0 & -348.7 & 163.6 & $\mathrm{x}$ & 0 & 570.9 \\
\hline $\mathrm{Sc}^{3+}$ & -645.6 & -525.1 & -382.0 & 332.1 & $\mathrm{x}$ & 0 & $\mathrm{x}$ \\
\hline $\mathrm{Sm}^{3+}$ & -673.6 & -367.6 & -314.2 & $\mathrm{x}$ & $\mathrm{x}$ & 0 & 479.1 \\
\hline $\mathrm{Tb}^{3+}$ & -596.8 & -387.0 & -343.1 & $\mathrm{x}$ & $\mathrm{x}$ & 0 & 1043.5 \\
\hline $\mathrm{Tl}^{3+}$ & -325.1 & -277.8 & -215.9 & 6.3 & $\mathrm{x}$ & 0 & $\mathrm{x}$ \\
\hline $\mathrm{Tm}^{3+}$ & -693.7 & -414.7 & -379.5 & $\mathrm{x}$ & $\mathrm{x}$ & 0 & $\mathrm{x}$ \\
\hline $\mathrm{Y}^{3+}$ & -766.5 & -723.0 & -397.9 & 348.5 & $\mathrm{x}$ & 0 & $\mathrm{x}$ \\
\hline $\mathrm{Yb}^{3+}$ & -762.7 & -430.9 & -415.9 & $\mathrm{x}$ & $\mathrm{x}$ & 0 & $\mathrm{x}$ \\
\hline
\end{tabular}

$\mathrm{x}=$ not enough data available

Case 2: divalent and trivalent metal with different univalent anion

$(1-\mathrm{x}) \mathrm{M}^{2+}\left(\mathrm{B}^{-}\right)_{2}+(\mathrm{x}) \mathrm{M}^{3+}\left(\mathrm{C}^{-}\right)_{3}+2 \mathrm{OH}^{-}+(\mathrm{x} / \mathrm{n}) \mathrm{A}^{\mathrm{n}-}=$

$$
\left[\mathrm{M}^{2+}{ }_{1-\mathrm{x}} \mathrm{M}_{\mathrm{x}}^{3+}(\mathrm{OH})_{2}\right] \mathrm{A}_{\mathrm{x} / \mathrm{n}}^{\mathrm{n}-}+2(1-\mathrm{x}) \mathrm{B}^{-}+3(\mathrm{x}) \mathrm{C}^{-}
$$

A similar analysis as in Case 1 gives the Gibbs free energy change of reaction:

$$
\begin{aligned}
& \Delta_{\mathrm{CP}} \mathrm{G}_{\mathrm{m}}^{\mathrm{o}}\{\mathrm{T}, \mathrm{LDH}\}=(1-\mathrm{x})\left[\Delta_{\mathrm{f}} \mathrm{G}_{\mathrm{m}}^{\mathrm{o}}\left\{\mathrm{T}, \mathrm{M}^{2+}(\mathrm{OH})_{2}\right\}-\Delta_{\mathrm{f}} \mathrm{G}_{\mathrm{m}}^{\mathrm{o}}\left\{\mathrm{T}, \mathrm{M}^{2+}\left(\mathrm{B}^{-}\right)_{2}\right\}\right] \\
& +(\mathrm{x})\left[\Delta_{\mathrm{f}} \mathrm{G}_{\mathrm{m}}^{\mathrm{o}}\left\{\mathrm{T}, \mathrm{M}^{3+}(\mathrm{OH})_{3}\right\}-\Delta_{\mathrm{f}} \mathrm{G}_{\mathrm{m}}^{\mathrm{o}}\left\{\mathrm{T}, \mathrm{M}^{3+}\left(\mathrm{C}^{-}\right)_{3}\right\}\right]+ \\
& \quad(\mathrm{x})\left[\Delta_{\mathrm{C}} \mathrm{G}_{\mathrm{m}}^{\mathrm{o}}\left\{\mathrm{T}, \mathrm{M}^{2+} \mathrm{A}\right\}\right]+(2)\left[\Delta_{\mathrm{f}} \mathrm{G}_{\mathrm{m}}^{\mathrm{o}}\left\{\mathrm{T}, \mathrm{B}^{-}\right\}-\Delta_{\mathrm{f}} \mathrm{G}_{\mathrm{m}}^{\mathrm{o}}\left\{\mathrm{T}, \mathrm{OH}^{-}\right\}\right]+ \\
& \quad(\mathrm{x})\left[3 \Delta_{\mathrm{f}} \mathrm{G}_{\mathrm{m}}^{\mathrm{o}}\left\{\mathrm{T}, \mathrm{C}^{-}\right\}-2 \Delta_{\mathrm{f}} \mathrm{G}_{\mathrm{m}}^{\mathrm{o}}\left\{\mathrm{T}, \mathrm{B}^{-}\right\}-\Delta_{\mathrm{f}} \mathrm{G}_{\mathrm{m}}^{\mathrm{o}}\left\{\mathrm{T}, \mathrm{OH}^{-}\right\}\right]
\end{aligned}
$$

Equation 30 is similar to Equation 28. Results for the fifth contribution term are shown in Table 6. For example, the $\mathrm{Cl}^{-}-\mathrm{NO}_{3}$ $\left(\mathrm{B}^{-} \mathrm{C}^{-}\right)$fifth contribution term to the standard Gibbs free energy is given by:
$3 \Delta_{\mathrm{f}} \mathrm{G}_{\mathrm{m}}^{\mathrm{o}}\left\{298.15 \mathrm{~K}, \mathrm{NO}_{3}^{-}\right\}-2 \Delta_{\mathrm{f}} \mathrm{G}_{\mathrm{m}}^{\mathrm{o}}\left\{298.15 \mathrm{~K}, \mathrm{Cl}^{-}\right\}-\Delta_{\mathrm{f}} \mathrm{G}_{\mathrm{m}}^{\mathrm{o}}\{298.15 \mathrm{~K}$, $\left.\mathrm{OH}^{-}\right\}=3(-111.4)-2(-131.3)-(-157.3)=85.6 \mathrm{~kJ} \mathrm{~mol}^{-1}$

Case 3: divalent metal-univalent anion and trivalent metaldivalent anion

$$
\begin{gathered}
(1-\mathrm{x}) \mathrm{M}^{2+}\left(\mathrm{B}^{-}\right)_{2}+(\mathrm{x} / 2) \mathrm{M}^{3+}{ }_{2}\left(\mathrm{D}^{2-}\right)_{3}+2 \mathrm{OH}^{-}+(\mathrm{x} / \mathrm{n}) \mathrm{A}^{\mathrm{n}-}= \\
{\left[\mathrm{M}^{2+}{ }_{1-\mathrm{x}} \mathrm{M}^{3+}{ }_{\mathrm{x}}(\mathrm{OH})_{2}\right] \mathrm{A}^{\mathrm{n}-}{ }_{\mathrm{x} / \mathrm{n}}+2(1-\mathrm{x}) \mathrm{B}^{-}+(3 \mathrm{x} / 2) \mathrm{D}^{2-}}
\end{gathered}
$$

\begin{tabular}{|c|c|c|c|c|c|c|c|}
\hline \multirow[b]{2}{*}{$\mathrm{B}^{-}$} & \multicolumn{6}{|c|}{ Contribution Term, $\mathrm{kJ} \mathrm{mol}^{-1}, \mathrm{C}^{-}$} & \multirow{2}{*}{$\frac{\mathrm{D}^{2-}, \text { Equation } 32}{\mathrm{SO}_{4}{ }^{2-}}$} \\
\hline & $\mathrm{I}^{-}$ & $\mathrm{Br}^{-}$ & $\mathrm{Cl}^{-}$ & $\mathrm{NO}_{3}^{-}$ & $\mathrm{F}^{-}$ & $\mathrm{OH}^{-}$ & \\
\hline $\mathrm{I}^{-}$ & 105.7 & -52.2 & -133.3 & -73.8 & -579.5 & -211.4 & -855.3 \\
\hline $\mathrm{Br}^{-}$ & 211.0 & 53.1 & -28.0 & 31.5 & -474.2 & -106.1 & -750.0 \\
\hline $\mathrm{Cl}^{-}$ & 265.0 & 107.1 & 26.0 & 85.6 & -420.2 & -52.1 & -695.9 \\
\hline $\mathrm{NO}_{3}^{-}$ & 225.4 & 67.5 & -13.6 & 45.9 & -459.8 & -91.7 & -735.6 \\
\hline $\mathrm{F}^{-3}$ & 562.5 & 404.6 & 323.5 & 383.0 & -122.7 & 245.4 & -398.5 \\
\hline $\mathrm{OH}^{-}$ & 317.1 & 159.2 & 78.1 & 137.6 & -368.1 & 0.0 & -643.9 \\
\hline
\end{tabular}

The standard Gibbs free energy change of reaction is:

$$
\begin{aligned}
& \Delta_{\mathrm{CP}} \mathrm{G}_{\mathrm{m}}^{\mathrm{o}}\{\mathrm{T}, \mathrm{LDH}\}=(1-\mathrm{x})\left[\Delta_{\mathrm{f}} \mathrm{G}_{\mathrm{m}}^{\mathrm{o}}\left\{\mathrm{T}, \mathrm{M}^{2+}(\mathrm{OH})_{2}\right\}-\Delta_{\mathrm{f}} \mathrm{G}_{\mathrm{m}}^{\mathrm{o}}\left\{\mathrm{T}, \mathrm{M}^{2+}\left(\mathrm{B}^{-}\right)_{2}\right\}\right] \\
& +(\mathrm{x})\left[\Delta_{\mathrm{f}} \mathrm{G}_{\mathrm{m}}^{\mathrm{o}}\left\{\mathrm{T}, \mathrm{M}^{3+}(\mathrm{OH})_{3}\right\}-(1 / 2) \Delta_{\mathrm{f}} \mathrm{G}_{\mathrm{m}}^{\mathrm{o}}\left\{\mathrm{T}, \mathrm{M}^{3+}{ }_{2}\left(\mathrm{D}^{2-}\right)_{3}\right\}\right]+ \\
& \quad(\mathrm{x})\left[\Delta_{\mathrm{C}} \mathrm{G}_{\mathrm{m}}^{\mathrm{o}}\left\{\mathrm{T}, \mathrm{M}^{2+} \mathrm{A}\right\}\right]+(2)\left[\Delta_{\mathrm{f}} \mathrm{G}_{\mathrm{m}}^{\mathrm{o}}\left\{\mathrm{T}, \mathrm{B}^{-}\right\}-\Delta_{\mathrm{f}} \mathrm{G}_{\mathrm{m}}^{\mathrm{o}}\left\{\mathrm{T}, \mathrm{OH}^{-}\right\}\right]+ \\
& \quad(\mathrm{x})\left[(3 / 2) \Delta_{\mathrm{f}} \mathrm{G}_{\mathrm{m}}^{\mathrm{o}}\left\{\mathrm{T}, \mathrm{D}^{2-}\right\}-2 \Delta_{\mathrm{f}} \mathrm{G}_{\mathrm{m}}^{\mathrm{o}}\left\{\mathrm{T}, \mathrm{B}^{-}\right\}-\Delta_{\mathrm{f}} \mathrm{G}_{\mathrm{m}}^{\mathrm{o}}\left\{\mathrm{T}, \mathrm{OH}^{-}\right\}\right]
\end{aligned}
$$

Contribution terms are defined as in Case 1. The contribution due to the difference in the anion of the trivalent metal from the

Table 6. Standard molar Gibbs free energy contribution term of trivalent metal anions in solution for Equation 30 at $298.15 \mathrm{~K}$ 
trivalent metal hydroxide (second term) is given in the last column of Table 5. Results of the free energy contribution of the trivalent metal anion in solution, for Equation 32, are shown in the last column of Table 6. Calculations are straightforward. For instance, the $\mathrm{NO}_{3}^{--}$ $\mathrm{SO}_{4}^{2-}\left(\mathrm{B}^{-}-\mathrm{D}^{2-}\right)$ fifth contribution term to the standard Gibbs free energy is obtained by:

$(3 / 2) \Delta_{\mathrm{f}} \mathrm{G}_{\mathrm{m}}^{\mathrm{o}}\left\{298.15 \mathrm{~K}, \mathrm{SO}_{4}^{2-}\right\}-2 \Delta_{\mathrm{f}} \mathrm{G}_{\mathrm{m}}^{\mathrm{o}}\left\{298.15 \mathrm{~K}, \mathrm{NO}_{3}^{-}\right\}-\Delta_{\mathrm{f}} \mathrm{G}_{\mathrm{m}}^{\mathrm{o}}\{298.15 \mathrm{~K}$, $\left.\mathrm{OH}^{-}\right\}=(3 / 2)(-743.8)-2(-111.4)-(-157.3)=-735.6 \mathrm{~kJ} \mathrm{~mol}^{-1}$

Case 4: divalent metal-divalent anion and trivalent metalunivalent anion

$$
\begin{aligned}
& (1-\mathrm{x}) \mathrm{M}^{2+}\left(\mathrm{D}^{2-}\right)+(\mathrm{x}) \mathrm{M}^{3+}\left(\mathrm{B}^{-}\right)_{3}+2 \mathrm{OH}^{-}+(\mathrm{x} / \mathrm{n}) \mathrm{A}^{\mathrm{n}-}= \\
& {\left[\mathrm{M}^{2+}{ }_{1-\mathrm{x}} \mathrm{M}^{3+}{ }_{\mathrm{x}}(\mathrm{OH})_{2}\right] \mathrm{A}^{\mathrm{n}-}{ }_{\mathrm{x} / \mathrm{n}}+(1-\mathrm{x}) \mathrm{D}^{2-}+3(\mathrm{x}) \mathrm{B}^{-}}
\end{aligned}
$$

and

$$
\begin{aligned}
\Delta_{\mathrm{CP}} \mathrm{G}_{\mathrm{m}}^{\mathrm{o}} & \{\mathrm{T}, \mathrm{LDH}\}=(1-\mathrm{x})\left[\Delta_{\mathrm{f}} \mathrm{G}_{\mathrm{m}}^{\mathrm{o}}\left\{\mathrm{T}, \mathrm{M}^{2+}(\mathrm{OH})_{2}\right\}-\Delta_{\mathrm{f}} \mathrm{G}_{\mathrm{m}}^{\mathrm{o}}\left\{\mathrm{T}, \mathrm{M}^{2+}\left(\mathrm{D}^{2-}\right)\right\}\right] \\
& +(\mathrm{x})\left[\Delta_{\mathrm{f}} \mathrm{G}_{\mathrm{m}}^{\mathrm{o}}\left\{\mathrm{T}, \mathrm{M}^{3+}(\mathrm{OH})_{3}\right\}-\Delta_{\mathrm{f}} \mathrm{G}_{\mathrm{m}}^{\mathrm{o}}\left\{\mathrm{T}, \mathrm{M}^{3+}\left(\mathrm{B}^{-}\right)_{3}\right\}\right]+ \\
& (\mathrm{x})\left[\Delta_{\mathrm{C}} \mathrm{G}_{\mathrm{m}}^{\mathrm{o}}\left\{\mathrm{T}, \mathrm{M}^{2+} \mathrm{A}\right\}\right]+(2)\left[(1 / 2) \Delta_{\mathrm{f}} \mathrm{G}_{\mathrm{m}}^{\mathrm{o}}\left\{\mathrm{T}, \mathrm{D}^{2-}\right\}-\right. \\
& \left.\Delta_{\mathrm{f}} \mathrm{G}_{\mathrm{m}}^{\mathrm{o}}\left\{\mathrm{T}, \mathrm{OH}^{-}\right\}\right]+(\mathrm{x})\left[3 \Delta_{\mathrm{f}} \mathrm{G}_{\mathrm{m}}^{\mathrm{o}}\left\{\mathrm{T}, \mathrm{B}^{-}\right\}-\Delta_{\mathrm{f}} \mathrm{G}_{\mathrm{m}}^{\mathrm{o}}\left\{\mathrm{T}, \mathrm{D}^{2-}\right\}-\right. \\
& \left.\Delta_{\mathrm{f}} \mathrm{G}_{\mathrm{m}}^{\mathrm{o}}\left\{\mathrm{T}, \mathrm{OH}^{-}\right\}\right]
\end{aligned}
$$

The first and fourth contribution term of Equation 34 are shown in the last two columns and last row of Table 4, respectively. Some results for the Gibbs free energy contribution of the trivalent metal anion in solution, fifth contribution term of Equation 34, are given in Table 7. Calculations are similar to the ones used in case 3 for the $\left(\mathrm{B}^{-}-\mathrm{D}^{2-}\right)$ fifth contribution term.
Case 5: divalent and trivalent metal with the same divalent anion

$$
\begin{gathered}
(1-\mathrm{x}) \mathrm{M}^{2+}\left(\mathrm{D}^{2-}\right)+(\mathrm{x} / 2) \mathrm{M}^{3+}{ }_{2}\left(\mathrm{D}^{2-}\right)_{3}+2 \mathrm{OH}^{-}+(\mathrm{x} / \mathrm{n}) \mathrm{A}^{\mathrm{n}-}= \\
{\left[\mathrm{M}^{2+}{ }_{1-\mathrm{x}} \mathrm{M}^{3+}{ }_{\mathrm{x}}(\mathrm{OH})_{2}\right] \mathrm{A}^{\mathrm{n}-}{ }_{\mathrm{x} / \mathrm{n}}+(1+\mathrm{x} / 2) \mathrm{D}^{2-}}
\end{gathered}
$$

and

$$
\begin{aligned}
\Delta_{\mathrm{CP}} \mathrm{G}_{\mathrm{m}}^{\mathrm{o}} & \{\mathrm{T}, \mathrm{LDH}\}=(1-\mathrm{x})\left[\Delta_{\mathrm{f}} \mathrm{G}_{\mathrm{m}}^{\mathrm{o}}\left\{\mathrm{T}, \mathrm{M}^{2+}(\mathrm{OH})_{2}\right\}-\Delta_{\mathrm{f}} \mathrm{G}_{\mathrm{m}}^{\mathrm{o}}\left\{\mathrm{T}, \mathrm{M}^{2+}\left(\mathrm{D}^{2-}\right)\right\}\right] \\
& +(\mathrm{x})\left[\Delta_{\mathrm{f}} \mathrm{G}_{\mathrm{m}}^{\mathrm{o}}\left\{\mathrm{T}, \mathrm{M}^{3+}(\mathrm{OH})_{3}\right\}-(1 / 2)_{\mathrm{f}} \mathrm{G}_{\mathrm{m}}^{\mathrm{o}}\left\{\mathrm{T}, \mathrm{M}^{3+}\left(\mathrm{D}^{2-}\right)_{3}\right\}\right]+ \\
& (\mathrm{x})\left[\Delta_{\mathrm{C}} \mathrm{G}_{\mathrm{m}}^{\mathrm{o}}\left\{\mathrm{T}, \mathrm{M}^{2+} \mathrm{A}\right\}+(2)\left[(1 / 2) \Delta_{\mathrm{f}} \mathrm{G}_{\mathrm{m}}^{\mathrm{o}}\left\{\mathrm{T}, \mathrm{D}^{2-}\right\}-\right.\right. \\
& \left.\Delta_{\mathrm{f}} \mathrm{G}_{\mathrm{m}}^{\mathrm{o}}\left\{\mathrm{T}, \mathrm{OH}^{-}\right\}\right]+(\mathrm{x})\left[(1 / 2) \Delta_{\mathrm{f}} \mathrm{G}_{\mathrm{m}}^{\mathrm{o}}\left\{\mathrm{T}, \mathrm{D}^{2-}\right\}-\Delta_{\mathrm{f}} \mathrm{G}_{\mathrm{m}}^{\mathrm{o}}\left\{\mathrm{T}, \mathrm{OH}^{-}\right\}\right](36)
\end{aligned}
$$

\begin{tabular}{|c|c|c|c|c|c|c|}
\hline \multirow[b]{2}{*}{$\mathrm{D}^{2-}$} & \multicolumn{6}{|c|}{ Contribution Term, $\mathrm{kJ} \mathrm{mol}^{-1}, \mathrm{~B}^{-}$} \\
\hline & $\mathrm{I}^{-}$ & $\mathrm{Br}$ & $\mathrm{Cl}^{-}$ & $\mathrm{NO}_{3}^{-}$ & $\mathrm{F}^{-}$ & $\mathrm{OH}^{-}$ \\
\hline $\mathrm{SO}_{4}^{2-}$ & 746.4 & 588.5 & 507.3 & 566.9 & 61.1 & 429.2 \\
\hline $\mathrm{CO}_{3}^{2-}$ & 530.4 & 372.5 & 291.4 & 350.9 & -154.8 & 213.3 \\
\hline
\end{tabular}

\begin{tabular}{|c|c|c|c|c|c|c|c|}
\hline Case & $\begin{array}{c}\mathrm{M}^{2+} \\
\text { Anion }\end{array}$ & $\begin{array}{c}\mathrm{M}^{3+} \\
\text { Anion }\end{array}$ & $\begin{array}{l}\text { CT1: } \text { M }^{2+} \text { salt } \\
\text { Table } 4\end{array}$ & $\begin{array}{l}\text { CT2: } \mathrm{M}^{3+} \text { salt } \\
\text { Table } 5\end{array}$ & $\begin{array}{c}\text { CT3: LDH-A } \\
\text { Table } 3\end{array}$ & $\begin{array}{l}\text { CT4: anions in } \\
\text { solution } \\
\text { from } \mathrm{M}^{2+} \text { salt }\end{array}$ & $\begin{array}{l}\text { CT5: anions in } \\
\text { solution } \\
\text { from } \mathrm{M}^{3+} \text { salt }\end{array}$ \\
\hline 1 Equation 28 & $\mathrm{~B}^{-}$ & $\mathrm{B}^{-}$ & $\begin{array}{c}\Delta_{\mathrm{f}} \mathrm{G}_{\mathrm{m}}^{\mathrm{o}}\left\{\mathrm{T}, \mathrm{M}^{2+}(\mathrm{OH})_{2}\right\}- \\
\Delta_{\mathrm{f}} \mathrm{G}_{\mathrm{m}}^{\mathrm{o}}\left\{\mathrm{T}, \mathrm{M}^{2+}\left(\mathrm{B}^{-}\right)_{2}\right\}\end{array}$ & $\begin{array}{c}\Delta_{\mathrm{f}} \mathrm{G}_{\mathrm{m}}^{\mathrm{o}}\left\{\mathrm{T}, \mathrm{M}^{3+}(\mathrm{OH})_{3}\right\}- \\
\Delta_{\mathrm{f}} \mathrm{G}_{\mathrm{m}}^{\mathrm{o}}\left\{\mathrm{T}, \mathrm{M}^{3+}\left(\mathrm{B}^{-}\right)_{3}\right\}\end{array}$ & $\Delta_{\mathrm{C}} \mathrm{G}_{\mathrm{T}}^{\mathrm{o}} \mathrm{M}^{2+} \mathrm{A}$ & $\begin{array}{c}\Delta_{\mathrm{f}} \mathrm{G}_{\mathrm{m}}^{\mathrm{o}}\left\{\mathrm{T}, \mathrm{B}^{-}\right\}- \\
\Delta_{\mathrm{f}} \mathrm{G}_{\mathrm{m}}^{\mathrm{o}}\left\{\mathrm{T}, \mathrm{OH}^{-}\right\} \\
\text {Table } 4\end{array}$ & $\begin{array}{c}\Delta_{\mathrm{f}} \mathrm{G}_{\mathrm{m}}^{\mathrm{o}}\left\{\mathrm{T}, \mathrm{B}^{-}\right\}- \\
\Delta_{\mathrm{f}} \mathrm{G}_{\mathrm{m}}^{\mathrm{o}}\left\{\mathrm{T}, \mathrm{OH}^{-}\right\} \\
\text {Table } 4\end{array}$ \\
\hline 2 Equation 30 & $\mathrm{~B}^{-}$ & $\mathrm{C}^{-}$ & $\begin{array}{c}\Delta_{\mathrm{f}} \mathrm{G}_{\mathrm{m}}^{\mathrm{o}}\left\{\mathrm{T}, \mathrm{M}^{2+}(\mathrm{OH})_{2}\right\}- \\
\Delta_{\mathrm{f}} \mathrm{G}_{\mathrm{m}}^{\mathrm{o}}\left\{\mathrm{T}, \mathrm{M}^{2+}\left(\mathrm{B}^{-}\right)_{2}\right\}\end{array}$ & $\begin{array}{c}\Delta_{\mathrm{f}} \mathrm{G}_{\mathrm{m}}^{\mathrm{o}}\left\{\mathrm{T}, \mathrm{M}^{3+}(\mathrm{OH})_{3}\right\}- \\
\Delta_{\mathrm{f}} \mathrm{G}_{\mathrm{m}}^{\mathrm{o}}\left\{\mathrm{T}, \mathrm{M}^{3+}\left(\mathrm{C}^{-}\right)_{3}\right\}\end{array}$ & $\Delta_{\mathrm{C}} \mathrm{G}_{\mathrm{T}}^{\mathrm{o}} \mathbf{M}^{2+} \mathrm{A}$ & $\begin{array}{c}\Delta_{\mathrm{f}} \mathrm{G}_{\mathrm{m}}^{\mathrm{o}}\left\{\mathrm{T}, \mathrm{B}^{-}\right\}- \\
\Delta_{\mathrm{f}} \mathrm{G}_{\mathrm{m}}^{\mathrm{o}}\left\{\mathrm{T}, \mathrm{OH}^{-}\right\} \\
\text {Table } 4\end{array}$ & $\begin{array}{c}3 \Delta_{\mathrm{f}} \mathrm{G}_{\mathrm{m}}^{\mathrm{o}}\left\{\mathrm{T}, \mathrm{C}^{-}\right\}- \\
2 \Delta_{\mathrm{f}} \mathrm{G}_{\mathrm{m}}^{\circ}\left\{\mathrm{T}, \mathrm{B}^{-}\right\}- \\
\Delta_{\mathrm{f}} \mathrm{G}_{\mathrm{m}}^{\mathrm{o}}\left\{\mathrm{T}, \mathrm{OH}^{-}\right\} \\
\text {Table } 6\end{array}$ \\
\hline 3 Equation 32 & $\mathrm{~B}^{-}$ & $\mathrm{D}^{2-}$ & $\begin{array}{c}\Delta_{\mathrm{f}} \mathrm{G}_{\mathrm{m}}^{\mathrm{o}}\left\{\mathrm{T}, \mathrm{M}^{2+}(\mathrm{OH})_{2}\right\}- \\
\Delta_{\mathrm{f}} \mathrm{G}_{\mathrm{m}}^{\mathrm{o}}\left\{\mathrm{T}, \mathrm{M}^{2+}\left(\mathrm{B}^{-}\right)_{2}\right\}\end{array}$ & $\begin{array}{c}\Delta_{\mathrm{f}} \mathrm{G}_{\mathrm{m}}^{\mathrm{o}}\left\{\mathrm{T}, \mathrm{M}^{3+}(\mathrm{OH})_{3}\right\}- \\
(1 / 2) \Delta_{\mathrm{f}} \mathrm{G}_{\mathrm{m}}^{\mathrm{o}}\left\{\mathrm{T}, \mathrm{M}_{2}^{3+}\left(\mathrm{D}^{2-}\right)_{3}\right\}\end{array}$ & $\Delta_{\mathrm{C}} \mathrm{G}_{\mathrm{T}}^{\mathrm{o}} \mathrm{M}^{2+} \mathrm{A}$ & $\begin{array}{c}\Delta_{\mathrm{f}} \mathrm{G}_{\mathrm{m}}^{\mathrm{o}}\left\{\mathrm{T}, \mathrm{B}^{-}\right\}- \\
\Delta_{\mathrm{f}} \mathrm{G}_{\mathrm{m}}^{\mathrm{o}}\left\{\mathrm{T}, \mathrm{OH}^{-}\right\} \\
\text {Table } 4\end{array}$ & $\begin{array}{c}(3 / 2) \Delta_{\mathrm{f}} \mathrm{G}_{\mathrm{m}}^{\mathrm{o}}\left\{\mathrm{T}, \mathrm{D}^{2-}\right\} \\
-2 \Delta_{\mathrm{f}} \mathrm{G}_{\mathrm{m}}^{\circ}\left\{\mathrm{T}, \mathrm{B}^{-}\right\} \\
-\Delta_{\mathrm{f}} \mathrm{G}_{\mathrm{m}}^{\mathrm{o}}\left\{\mathrm{T}, \mathrm{OH}^{-}\right\} \\
\text {Table } 6\end{array}$ \\
\hline 4 Equation 34 & $\mathrm{D}^{2-}$ & $\mathrm{B}^{-}$ & $\begin{array}{c}\Delta_{\mathrm{f}} \mathrm{G}_{\mathrm{m}}^{\mathrm{o}}\left\{\mathrm{T}, \mathrm{M}^{2+}(\mathrm{OH})_{2}\right\}- \\
\Delta_{\mathrm{f}} \mathrm{G}_{\mathrm{m}}^{\mathrm{o}}\left\{\mathrm{T}, \mathrm{M}^{2+}\left(\mathrm{D}^{2-}\right)\right\}\end{array}$ & $\begin{array}{c}\Delta_{\mathrm{f}} \mathrm{G}_{\mathrm{m}}^{\mathrm{o}}\left\{\mathrm{T}, \mathrm{M}^{3+}(\mathrm{OH})_{3}\right\}- \\
\Delta_{\mathrm{f}} \mathrm{G}_{\mathrm{m}}^{\mathrm{o}}\left\{\mathrm{T}, \mathrm{M}^{3+}\left(\mathrm{B}^{-}\right)_{3}\right\}\end{array}$ & $\Delta_{\mathrm{C}} \mathrm{G}_{\mathrm{T}}^{\mathrm{o}} \mathbf{M}^{2+} \mathrm{A}$ & $\begin{array}{c}(1 / 2) \Delta_{\mathrm{f}} \mathrm{G}_{\mathrm{m}}^{\mathrm{o}}\left\{\mathrm{T}, \mathrm{D}^{2-}\right\} \\
-\Delta_{\mathrm{f}} \mathrm{G}_{\mathrm{m}}^{\mathrm{o}}\left\{\mathrm{T}, \mathrm{OH}^{-}\right\} \\
\text {Table } 4\end{array}$ & $\begin{array}{c}3 \Delta_{\mathrm{f}} \mathrm{G}_{\mathrm{m}}^{\mathrm{o}}\left\{\mathrm{T}, \mathrm{B}^{-}\right\}- \\
\Delta_{\mathrm{f}} \mathrm{G}_{\mathrm{m}}^{\mathrm{o}}\left\{\mathrm{T}, \mathrm{D}^{2-}\right\}- \\
\Delta_{\mathrm{f}} \mathrm{G}_{\mathrm{m}}^{\mathrm{o}}\left\{\mathrm{T}, \mathrm{OH}^{-}\right\} \\
\text {Table } 7\end{array}$ \\
\hline 5 Equation 36 & $\mathrm{D}^{2-}$ & $\mathrm{D}^{2-}$ & $\begin{array}{c}\Delta_{\mathrm{f}} \mathrm{G}_{\mathrm{m}}^{\mathrm{o}}\left\{\mathrm{T}, \mathrm{M}^{2+}(\mathrm{OH})_{2}\right\}- \\
\Delta_{\mathrm{f}} \mathrm{G}_{\mathrm{m}}^{\mathrm{o}}\left\{\mathrm{T}, \mathrm{M}^{2+}\left(\mathrm{D}^{2-}\right)\right\}\end{array}$ & $\begin{array}{c}\Delta_{\mathrm{f}} \mathrm{G}_{\mathrm{m}}^{\mathrm{o}}\left\{\mathrm{T}, \mathrm{M}^{3+}(\mathrm{OH})_{3}\right\}- \\
(1 / 2) \Delta_{\mathrm{f}} \mathrm{G}_{\mathrm{m}}^{\mathrm{o}}\left\{\mathrm{T}, \mathrm{M}_{2}^{3+}\left(\mathrm{D}^{2-}\right)_{3}\right\}\end{array}$ & $\Delta_{\mathrm{C}} \mathrm{G}_{\mathrm{T}}^{\mathrm{o}} \mathrm{M}^{2+} \mathrm{A}$ & $\begin{array}{c}(1 / 2) \Delta_{\mathrm{f}} \mathrm{G}_{\mathrm{m}}^{\mathrm{o}}\left\{\mathrm{T}, \mathrm{D}^{2-}\right\} \\
-\Delta_{\mathrm{f}} \mathrm{G}_{\mathrm{m}}^{\mathrm{o}}\left\{\mathrm{T}, \mathrm{OH}^{-}\right\} \\
\text {Table } 4\end{array}$ & $\begin{array}{c}(1 / 2) \Delta_{\mathrm{f}} \mathrm{G}_{\mathrm{m}}^{\mathrm{o}}\left\{\mathrm{T}, \mathrm{D}^{2-}\right\} \\
-\Delta_{\mathrm{f}} \mathrm{G}_{\mathrm{m}}^{\mathrm{o}}\left\{\mathrm{T}, \mathrm{OH}^{-}\right\} \\
\text {Table } 4\end{array}$ \\
\hline
\end{tabular}

Values of the first, second and third contribution terms are shown in the last column of Table 4, last column of Tables 5 and 3, respectively. Results for the fourth and fifth contribution terms are given in the last row of Table 4. A summary of all studied cases is given in Table 8, and some examples of standard Gibbs free energy calculations for the synthesis of LDHs by coprecipitation are given in Table 9.

Table 7. Standard molar Gibbs free energy contribution term of trivalent metal anion in solution for Equation 34 at $298.15 \mathrm{~K}$

Table 8. Summary of Gibbs free energy calculations for the synthesis of LDHs: $\left[\mathrm{M}^{2+}{ }_{1-\mathrm{x}} \mathrm{M}^{3+}{ }_{\mathrm{x}}(\mathrm{OH})_{2}\right] \mathrm{A}^{\mathrm{n}-}{ }_{\mathrm{x} / \mathrm{n}}$ by coprecipitation $\Delta_{\mathrm{CP}} \mathrm{G}_{\mathrm{m}}^{\mathrm{o}}\{\mathrm{T}, \mathrm{LDH}\}=$ $(1-\mathrm{x}) \mathrm{CT} 1+(\mathrm{x}) \mathrm{CT} 2+(\mathrm{x}) \mathrm{CT} 3+(2) \mathrm{CT} 4+(\mathrm{x}) \mathrm{CT} 5$ 
Table 9. Estimated standard molar Gibbs free energies of reaction for some LDHs synthesized by coprecipitation at $298.15 \mathrm{~K}$

\begin{tabular}{|c|c|c|c|c|c|c|c|c|c|}
\hline Case & $\begin{array}{c}\mathrm{M}^{2+} \\
\text { Anion }\end{array}$ & $\begin{array}{c}\mathrm{M}^{3+} \\
\text { Anion }\end{array}$ & $\begin{array}{c}\text { LDH-A } \\
x\end{array}$ & $\begin{array}{c}\text { CT1: } \\
\mathrm{M}^{2+} \text { salt } \\
(1-\mathrm{x}) \mathrm{CT} 1\end{array}$ & $\begin{array}{c}\text { CT2: } \\
\mathrm{M}^{3+} \text { salt } \\
(\mathrm{x}) \mathrm{CT} 2\end{array}$ & $\begin{array}{c}\text { CT3: } \\
\text { LDH-A } \\
\text { (x)CT3 }\end{array}$ & $\begin{array}{c}\text { CT4: } \\
\text { Anion M }{ }^{2+} \\
\text { (2)CT4 }\end{array}$ & $\begin{array}{c}\text { CT5: } \\
\text { Anion } \mathrm{M}^{3+} \\
\text { (x)CT5 }\end{array}$ & $\begin{array}{c}\Delta_{\mathrm{CP}} \mathrm{G}_{\mathrm{m}}^{\mathrm{o}} \\
\mathrm{kJ} \mathrm{mol}^{-1}\end{array}$ \\
\hline 1 & $\begin{array}{c}\mathrm{Mg} \\
\mathrm{Cl}\end{array}$ & $\begin{array}{l}\mathrm{Al} \\
\mathrm{Cl}\end{array}$ & $\begin{array}{c}\mathrm{Cl} \\
0.25\end{array}$ & -181.4 & -104.8 & 23.7 & 52.0 & 6.5 & -204.0 \\
\hline 1 & $\begin{array}{l}\mathrm{Zn} \\
\mathrm{Cl}\end{array}$ & $\begin{array}{l}\mathrm{Al} \\
\mathrm{Cl}\end{array}$ & $\begin{array}{c}\mathrm{Cl} \\
0.25\end{array}$ & -137.2 & -104.8 & 16.4 & 52.0 & 6.5 & -167.1 \\
\hline 1 & $\begin{array}{l}\mathrm{Ni} \\
\mathrm{Cl}\end{array}$ & $\begin{array}{l}\mathrm{Al} \\
\mathrm{Cl}\end{array}$ & $\begin{array}{c}\mathrm{Cl} \\
0.25\end{array}$ & -136.9 & -104.8 & 16.3 & 52.0 & 6.5 & -166.9 \\
\hline 1 & $\begin{array}{l}\mathrm{Mg} \\
\mathrm{NO}_{3}\end{array}$ & $\begin{array}{c}\mathrm{Al} \\
\mathrm{NO}_{3}\end{array}$ & $\begin{array}{l}\mathrm{NO}_{3} \\
0.25\end{array}$ & -183.8 & -94.5 & 19.2 & 91.8 & 11.5 & -155.8 \\
\hline 1 & $\begin{array}{c}\mathrm{Zn} \\
\mathrm{NO}_{3}\end{array}$ & $\begin{array}{c}\mathrm{Al}^{3} \\
\mathrm{NO}_{3}\end{array}$ & $\begin{array}{l}\mathrm{NO}_{3} \\
0.25\end{array}$ & -191.7 & -94.5 & 20.5 & 91.8 & 11.5 & -162.4 \\
\hline 1 & $\begin{array}{c}\mathrm{Ni} \\
\mathrm{NO}_{3}\end{array}$ & $\begin{array}{c}\mathrm{Al} \\
\mathrm{NO}_{3}\end{array}$ & $\begin{array}{l}\mathrm{NO}_{3} \\
0.25\end{array}$ & -154.4 & -94.5 & 14.3 & 91.8 & 11.5 & -131.3 \\
\hline 1 & $\begin{array}{l}\mathrm{Mg} \\
\mathrm{NO}_{3}\end{array}$ & $\begin{array}{c}\mathrm{Al} \\
\mathrm{NO}_{3}\end{array}$ & $\begin{array}{l}\mathrm{CO}_{3} \\
0.25\end{array}$ & -183.8 & -94.5 & 4.5 & 91.8 & 11.5 & -170.6 \\
\hline 1 & $\begin{array}{c}\mathrm{Zn} \\
\mathrm{NO}_{3}\end{array}$ & $\begin{array}{c}\mathrm{Al} \\
\mathrm{NO}_{3}\end{array}$ & $\begin{array}{l}\mathrm{CO}_{3} \\
0.25\end{array}$ & -191.7 & -94.5 & 4.2 & 91.8 & 11.5 & -178.7 \\
\hline 1 & $\begin{array}{c}\mathrm{Ni} \\
\mathrm{NO}_{3}\end{array}$ & $\begin{array}{c}\mathrm{Al} \\
\mathrm{NO}_{3} \\
\end{array}$ & $\begin{array}{l}\mathrm{CO}_{3} \\
0.25\end{array}$ & -154.4 & -94.5 & 5.4 & 91.8 & 11.5 & -140.2 \\
\hline
\end{tabular}

\section{SOLUBILITY OF LAYERED DOUBLE HYDROXIDES}

A general expression of the dissolution of LDHs in acidic media is given by:

$$
\begin{aligned}
{\left[\mathrm{M}^{2+}{ }_{1-\mathrm{x}} \mathrm{M}^{3+}(\mathrm{OH})_{2}\right]\left(\mathrm{A}^{\mathrm{n}-}\right)_{\mathrm{x} / \mathrm{n}}+2 \mathrm{H}^{+}(\mathrm{aq})=} \\
(1-\mathrm{x}) \mathrm{M}^{2+}(\mathrm{aq})+(\mathrm{x}) \mathrm{M}^{3+}(\mathrm{aq})+(\mathrm{x} / \mathrm{n}) \mathrm{A}^{\mathrm{n}-}+2 \mathrm{H}_{2} \mathrm{O}
\end{aligned}
$$
is:

The standard molar Gibbs free energy change of the dissolution

$$
\begin{aligned}
\Delta_{\mathrm{D}} \mathrm{G}_{\mathrm{m}}^{\mathrm{o}} & \{\mathrm{T}, \mathrm{LDH}\}=(1-\mathrm{x}) \Delta_{\mathrm{f}} \mathrm{G}_{\mathrm{m}}^{\mathrm{o}}\left\{\mathrm{T}, \mathrm{M}^{2+}\right\}+(\mathrm{x}) \Delta_{\mathrm{f}} \mathrm{G}_{\mathrm{m}}^{\mathrm{o}}\left\{\mathrm{T}, \mathrm{M}^{3+}\right]+ \\
& (\mathrm{x} / \mathrm{n}) \Delta_{\mathrm{f}} \mathrm{G}_{\mathrm{m}}^{\mathrm{o}}\left\{\mathrm{T}, \mathrm{A}^{\mathrm{n}-}\right\}+2 \Delta_{\mathrm{f}} \mathrm{G}_{\mathrm{m}}^{\mathrm{o}}\left\{\mathrm{T}, \mathrm{H}_{2} \mathrm{O}\right\}-\Delta_{\mathrm{f}} \mathrm{G}_{\mathrm{m}}^{\mathrm{o}}\{\mathrm{T}, \mathrm{LDH}\}- \\
& 2 \Delta_{\mathrm{f}} \mathrm{G}_{\mathrm{m}}^{\mathrm{o}}\left\{\mathrm{T}, \mathrm{H}^{+}\right\}
\end{aligned}
$$

Similarly to the synthesis of LDHs by H-R methods, depending on the LDH interlamellar anion, two cases are distinguished: 1) Hydroxyl anion $\left(\mathrm{A}^{\mathrm{n}-}=\mathrm{OH}^{-}\right)$and 2) Non-hydroxyl anions $\left(\mathrm{A}^{\mathrm{n}-} \neq \mathrm{OH}^{-}\right)$.

\section{Dissolution of meixnerite-like compounds}

Substituting $\mathrm{OH}^{-}$and Equation 6 into Equation 37 and taking into account that: $(\mathrm{x}) \mathrm{OH}^{-}+(\mathrm{x}) \mathrm{H}^{+}=(\mathrm{x}) \mathrm{H}_{2} \mathrm{O}$, then

$$
\begin{array}{r}
(1-\mathrm{x})\left[\mathrm{M}^{2+}(\mathrm{OH})_{2}+2 \mathrm{H}^{+}(\mathrm{aq})\right]+(\mathrm{x})\left[\mathrm{M}^{3+}(\mathrm{OH})_{3}+3 \mathrm{H}^{+}(\mathrm{aq})\right]= \\
(1-\mathrm{x})\left[\mathrm{M}^{2+}(\mathrm{aq})+2 \mathrm{H}_{2} \mathrm{O}\right]+(\mathrm{x})\left[\mathrm{M}^{3+}(\mathrm{aq})+3 \mathrm{H}_{2} \mathrm{O}\right]
\end{array}
$$

and

$$
\begin{aligned}
\Delta_{\mathrm{D}} \mathrm{G}_{\mathrm{m}}^{\mathrm{o}} & \{\mathrm{T}, \mathrm{MLC}\}=(1-\mathrm{x})\left[2 \Delta_{\mathrm{f}} \mathrm{G}_{\mathrm{m}}^{\mathrm{o}}\left\{\mathrm{T}, \mathrm{H}_{2} \mathrm{O}\right\}+\Delta_{\mathrm{f}} \mathrm{G}_{\mathrm{m}}^{\mathrm{o}}\left\{\mathrm{T}, \mathrm{M}^{2+}\right]-\right. \\
& \left.\Delta_{\mathrm{f}} \mathrm{G}_{\mathrm{m}}^{\mathrm{o}}\left\{\mathrm{T}, \mathrm{M}^{2+}(\mathrm{OH})_{2}\right\}\right]+(\mathrm{x})\left[3 \Delta_{\mathrm{f}} \mathrm{G}_{\mathrm{m}}^{\mathrm{o}}\left\{\mathrm{T}, \mathrm{H}_{2} \mathrm{O}\right\}+\Delta_{\mathrm{f}} \mathrm{G}_{\mathrm{m}}^{\mathrm{o}}\left\{\mathrm{T}, \mathrm{M}^{3+}\right\}-\right. \\
& \left.\Delta_{\mathrm{f}} \mathrm{G}_{\mathrm{m}}^{\circ}\left\{\mathrm{T}, \mathrm{M}^{3+}(\mathrm{OH})_{3}\right\}\right]
\end{aligned}
$$

Equation 40 is a linear combination of the dissolution reactions of the divalent and trivalent hydroxides:

$\mathrm{M}^{2+}(\mathrm{OH})_{2}+2 \mathrm{H}^{+}=\mathrm{M}^{2+}+2 \mathrm{H}_{2} \mathrm{O}$

$$
\begin{aligned}
& \Delta_{\mathrm{D}} \mathrm{G}_{\mathrm{m}}^{\mathrm{o}}\left\{\mathrm{T}, \mathrm{M}^{2+}(\mathrm{OH})_{2}\right\}=2 \Delta_{\mathrm{f}} \mathrm{G}_{\mathrm{m}}^{\mathrm{o}}\left\{\mathrm{T}, \mathrm{H}_{2} \mathrm{O}\right\}+\Delta_{\mathrm{f}} \mathrm{G}_{\mathrm{m}}^{\mathrm{o}}\left\{\mathrm{T}, \mathrm{M}^{2+}\right\}- \\
& \quad \Delta_{\mathrm{f}} \mathrm{G}_{\mathrm{m}}^{\mathrm{o}}\left\{\mathrm{T}, \mathrm{M}^{2+}(\mathrm{OH})_{2}\right\} \\
& \mathrm{M}^{3+}(\mathrm{OH})_{3}+3 \mathrm{H}^{+}=\mathrm{M}^{3+}+3 \mathrm{H}_{2} \mathrm{O} \\
& \Delta_{\mathrm{D}} \mathrm{G}_{\mathrm{m}}^{\mathrm{o}}\left\{\mathrm{T}, \mathrm{M}^{3+}(\mathrm{OH})_{3}\right\}=3 \Delta_{\mathrm{f}} \mathrm{G}_{\mathrm{m}}^{\mathrm{o}}\left\{\mathrm{T}, \mathrm{H}_{2} \mathrm{O}\right\}+\Delta_{\mathrm{f}} \mathrm{G}_{\mathrm{m}}^{\mathrm{o}}\left\{\mathrm{T}, \mathrm{M}^{3+}\right\}- \\
& \quad \Delta_{\mathrm{f}} \mathrm{G}_{\mathrm{m}}^{\mathrm{o}}\left\{\mathrm{T}, \mathrm{M}^{3+}(\mathrm{OH})_{3}\right\}
\end{aligned}
$$

Finally, Equation 40 is rewritten in terms of the standard Gibbs free energies of dissolution of metal hydroxides:

$$
\begin{gathered}
\Delta_{\mathrm{D}} \mathrm{G}_{\mathrm{m}}^{\mathrm{o}}\{\mathrm{T}, \mathrm{MLC}\}=(1-\mathrm{x}) \Delta_{\mathrm{D}} \mathrm{G}_{\mathrm{m}}^{\mathrm{o}}\left\{\mathrm{T}, \mathrm{M}^{2+}(\mathrm{OH})_{2}\right\}+ \\
(\mathrm{x}) \Delta_{\mathrm{D}} \mathrm{G}_{\mathrm{m}}^{\mathrm{o}}\left\{\mathrm{T}, \mathrm{M}^{3+}(\mathrm{OH})_{3}\right\}
\end{gathered}
$$

\section{Standard molar Gibbs free energies of dissolution of metal hydroxides at different temperatures}

Data for the standard molar Gibbs free energy change of dissolution at different temperatures are given in Figures 5 and 6. Results for the dissolution reactions of some $\mathrm{M}^{+}$and $\mathrm{M}^{2+}$ hydroxides can be seen in Figure 5. Some dissolution reactions of $\mathrm{M}^{3+}$ and $\mathrm{M}^{4+}$ hydroxides are also shown in Figure 6. All the calculations are based on Equations 41 and 43. A procedure similar to the one described for the Gibbs free energy of hydration for metal oxides at different temperatures is followed. Standard Gibbs free energy change of dissolution for many $\mathrm{M}^{+}, \mathrm{M}^{2+}, \mathrm{M}^{3+}$ and $\mathrm{M}^{4+}$ hydroxides are given in Tables 10 and 11. Again, information about the reactions, equations used, calculated $\Delta_{\mathrm{D}} \mathrm{G}_{\mathrm{m}}^{\mathrm{o}}\{\mathrm{T}\}$ 's and references for each of the entries are provided.

Data in Tables 10 and 11 together with Equation 45 are used to estimate the standard molar Gibbs free energy change of dissolution of MLCs in acidic media. These results are summarized schematically in Figure 7 as a nomogram, and some examples of MLCs are shown. Data from Figures 5 and 6 can be used for the estimation of the standard free energy change of dissolution at temperatures different from $298.15 \mathrm{~K}$. The procedure described before to use Figure 4 can be applied for Figure 7. 


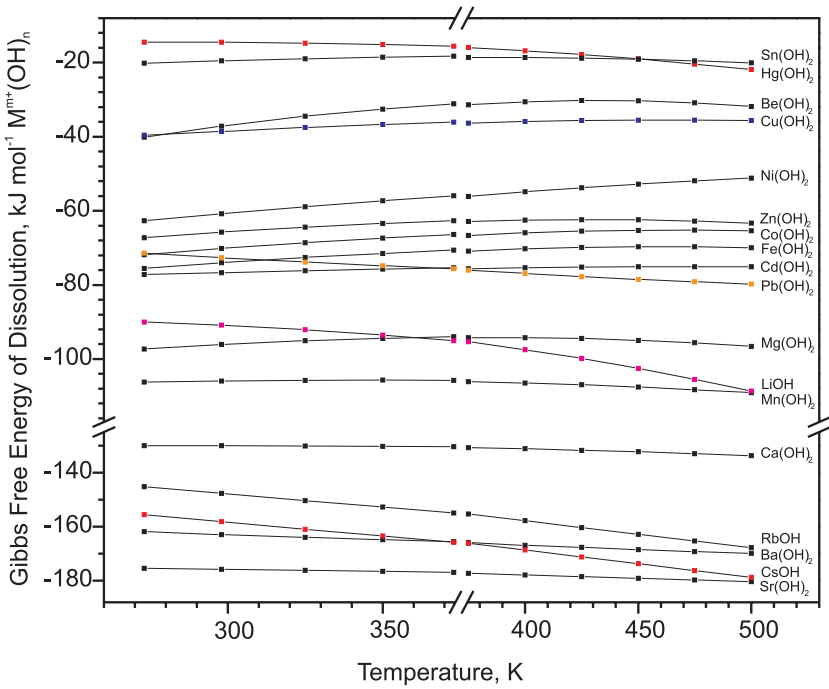

Figure 5. Standard molar Gibbs free energy change of dissolution for some $M^{+}$and $M^{2+}$ hydroxides at different temperatures

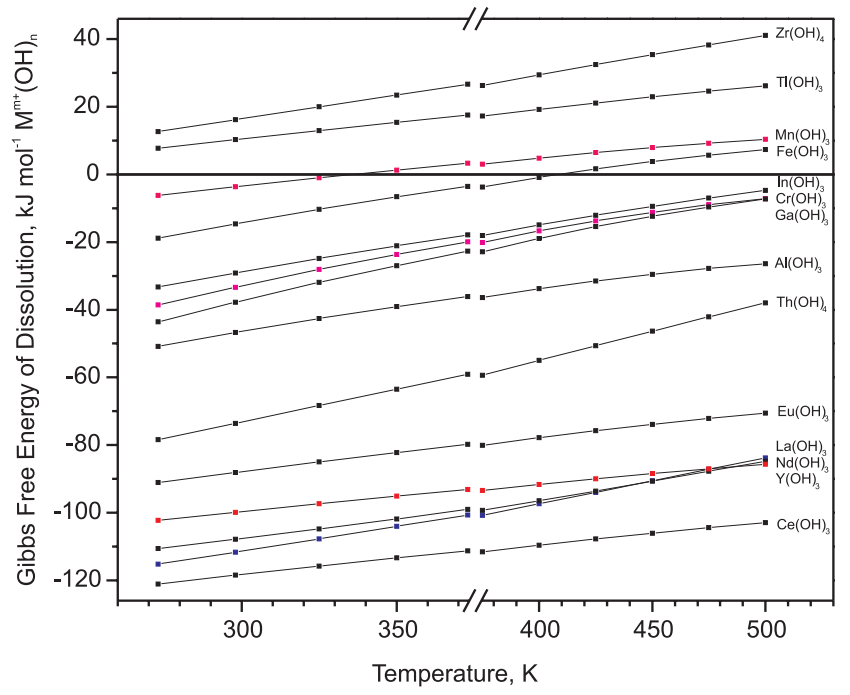

Figure 6. Standard molar Gibbs free energy change of dissolution for some $M^{3+}$ and $M^{4+}$ hydroxides at different temperatures

Table 10. Standard molar Gibbs free energy change of the dissolution reaction for some $\mathrm{M}^{+}$and $\mathrm{M}^{2+}$ hydroxides at $298.15 \mathrm{~K}$

\begin{tabular}{|c|c|c|c|}
\hline Dissolution Reaction & Equations & $\Delta_{\mathrm{D}} \mathrm{G}_{\mathrm{m}}^{\mathrm{o}}\left\{298.15 \mathrm{~K}, \mathrm{M}^{\mathrm{m}+}(\mathrm{OH})_{\mathrm{n}}\right\}, \mathrm{kJ} \mathrm{mol}^{-1}$ & Ref. \\
\hline $\mathrm{AgOH}(\mathrm{s})+\mathrm{H}^{+}(\mathrm{aq})=\mathrm{Ag}^{+}(\mathrm{aq})+\mathrm{H}_{2} \mathrm{O}$ & 20 & -35.3 & 86,89 \\
\hline $\mathrm{TlOH}(\mathrm{s})+\mathrm{H}^{+}(\mathrm{aq})=\mathrm{Tl}^{+}(\mathrm{aq})+\mathrm{H}_{2} \mathrm{O}^{2}$ & $19-20$ & -73.6 & $86,89,92$ \\
\hline $\mathrm{LiOH}(\mathrm{s})+\mathrm{H}^{+}(\mathrm{aq})=\mathrm{Li}^{+}(\mathrm{aq})+\mathrm{H}_{2}^{2} \mathrm{O}$ & $19-20$ & -90.8 & $86,88-89$ \\
\hline $\mathrm{NaOH}(\mathrm{s})+\mathrm{H}^{+}(\mathrm{aq})=\mathrm{Na}^{+}(\mathrm{aq})+\mathrm{H}_{2} \mathrm{O}$ & $19-20$ & -120.0 & 86,89 \\
\hline $\mathrm{RbOH}(\mathrm{s})+\mathrm{H}^{+}(\mathrm{aq})=\mathrm{Rb}^{+}(\mathrm{aq})+\mathrm{H}_{2}^{2} \mathrm{O}$ & 20 & -147.7 & $86,88,89$ \\
\hline $\mathrm{CsOH}(\mathrm{s})+\mathrm{H}^{+}(\mathrm{aq})=\mathrm{Cs}^{+}(\mathrm{aq})+\mathrm{H}_{2} \mathrm{O}$ & 19 & -158.2 & $86,88,89$ \\
\hline $\mathrm{Pt}(\mathrm{OH})_{2}(\mathrm{~s})+2 \mathrm{H}^{+}(\mathrm{aq})=\mathrm{Pt}^{2+}(\mathrm{aq})+2 \mathrm{H}_{2} \mathrm{O}$ & 20 & 39.0 & 87,89 \\
\hline $\mathrm{Pd}(\mathrm{OH})_{2}(\mathrm{~s})+2 \mathrm{H}^{+}(\mathrm{aq})=\mathrm{Pd}^{2+}(\mathrm{aq})+2 \mathrm{H}_{2} \mathrm{O}$ & 20 & 7.3 & 87,89 \\
\hline $\mathrm{Hg}(\mathrm{OH})_{2}(\mathrm{~s})+2 \mathrm{H}^{+}(\mathrm{aq})=\mathrm{Hg}^{2+}(\mathrm{aq})+2 \mathrm{H}_{2} \mathrm{O}$ & $19-20$ & -14.6 & 86,89 \\
\hline $\mathrm{Sn}(\mathrm{OH}),(\mathrm{s})+2 \mathrm{H}^{+}(\mathrm{aq})=\mathrm{Sn}^{2+}(\mathrm{aq})+2 \mathrm{H}_{2} \mathrm{O}^{2}$ & $19-20$ & -16.4 & $86,89,90,92$ \\
\hline $\mathrm{Be}(\mathrm{OH})_{2}(\mathrm{~s})+2 \mathrm{H}^{+}(\mathrm{aq})=\mathrm{Be}^{2+}(\mathrm{aq})+2 \mathrm{H}_{2} \mathrm{O}$ & $19-20$ & -36.9 & $86,88,89$ \\
\hline $\mathrm{Cu}(\mathrm{OH})_{2}(\mathrm{~s})+2 \mathrm{H}^{+}(\mathrm{aq})=\mathrm{Cu}^{2+}(\mathrm{aq})+2 \mathrm{H}_{2} \mathrm{O}$ & 20 & -51.4 & $85,86,89,90$ \\
\hline $\mathrm{Ni}(\mathrm{OH})_{2}(\mathrm{~s})+2 \mathrm{H}^{+}(\mathrm{aq})=\mathrm{Ni}^{2+}(\mathrm{aq})+2 \mathrm{H}_{2} \mathrm{O}$ & $19-20$ & -60.4 & $86,89,90,92$ \\
\hline $\mathrm{Zn}(\mathrm{OH})_{2}(\mathrm{~s})+2 \mathrm{H}^{+}(\mathrm{aq})=\mathrm{Zn}^{2+}(\mathrm{aq})+2 \mathrm{H}_{2} \mathrm{O}$ & $19-20$ & -65.8 & $86,89,90,92$ \\
\hline $\mathrm{Co}(\mathrm{OH})_{2}(\mathrm{~s})+2 \mathrm{H}^{+}(\mathrm{aq})=\mathrm{Co}^{2+}(\mathrm{aq})+2 \mathrm{H}_{2} \mathrm{O}$ & $19-20$ & -70.8 & $86,88,89$ \\
\hline $\mathrm{Pb}(\mathrm{OH})_{2}(\mathrm{~s})+2 \mathrm{H}^{+}(\mathrm{aq})=\mathrm{Pb}^{2+}(\mathrm{aq})+2 \mathrm{H}_{2} \mathrm{O}$ & $19-20$ & -71.2 & $86,89-90,92$ \\
\hline $\mathrm{Fe}(\mathrm{OH})_{2}(\mathrm{~s})+2 \mathrm{H}^{+}(\mathrm{aq})=\mathrm{Fe}^{2+}(\mathrm{aq})+2 \mathrm{H}_{2}^{2} \mathrm{O}$ & $19-20$ & -73.6 & $86,88,89$ \\
\hline $\mathrm{Cd}(\mathrm{OH})_{2}(\mathrm{~s})+2 \mathrm{H}^{+}(\mathrm{aq})=\mathrm{Cd}^{2+}(\mathrm{aq})+2 \mathrm{H}_{2}^{2} \mathrm{O}$ & $19-20$ & -76.5 & $85,86,89$ \\
\hline $\mathrm{Mg}(\mathrm{OH})_{2}(\mathrm{~s})+2 \mathrm{H}^{+}(\mathrm{aq})=\mathrm{Mg}^{2+}(\mathrm{aq})+2 \mathrm{H}_{2} \mathrm{O}$ & $19-20$ & -96.1 & 86,89 \\
\hline $\mathrm{Mn}(\mathrm{OH})_{2}(\mathrm{~s})+2 \mathrm{H}^{+}(\mathrm{aq})=\mathrm{Mn}^{2+}(\mathrm{aq})+2 \mathrm{H}_{2}^{2} \mathrm{O}$ & $19-20$ & -105.6 & $86,89,92$ \\
\hline $\mathrm{Ca}(\mathrm{OH})_{2}(\mathrm{~s})+2 \mathrm{H}^{+}(\mathrm{aq})=\mathrm{Ca}^{2+}(\mathrm{aq})+2 \mathrm{H}_{2} \mathrm{O}$ & $19-20$ & -130.1 & 86,89 \\
\hline $\mathrm{Ba}(\mathrm{OH})_{2}(\mathrm{~s})+2 \mathrm{H}^{+}(\mathrm{aq})=\mathrm{Ba}^{2+}(\mathrm{aq})+2 \mathrm{H}_{2} \mathrm{O}$ & $19-20$ & -147.4 & $86,88,89$ \\
\hline $\mathrm{Sr}(\mathrm{OH})_{2}(\mathrm{~s})+2 \mathrm{H}^{+}(\mathrm{aq})=\mathrm{Sr}^{2+}(\mathrm{aq})+2 \mathrm{H}_{2} \mathrm{O}$ & $19-20$ & -174.4 & $85,86,89$ \\
\hline
\end{tabular}

\section{Dissolution of LDHs with anions different from $\mathrm{OH}^{-}$}

Combining Equation 1 and Equation 37 and rearranging terms, the following expression is obtained:

$$
\begin{aligned}
& (1-\mathrm{x})\left[\mathrm{M}^{2+}(\mathrm{OH})_{2}+2 \mathrm{H}^{+}(\mathrm{aq})\right]+(\mathrm{x})\left[\mathrm{M}^{3+}(\mathrm{OH})_{3}+3 \mathrm{H}^{+}(\mathrm{aq})\right]+ \\
& (\mathrm{x} / 2) \mathrm{M}^{2+}\left(\mathrm{A}^{\mathrm{n}-}\right)_{2 / \mathrm{n}}+(\mathrm{x}) \mathrm{H}_{2} \mathrm{O}=(1-\mathrm{x})\left[\mathrm{M}^{2+}(\mathrm{aq})+2 \mathrm{H}_{2} \mathrm{O}\right]+ \\
& (\mathrm{x})\left[\mathrm{M}^{3+}(\mathrm{aq})+3 \mathrm{H}_{2} \mathrm{O}\right]+(\mathrm{x} / \mathrm{n}) \mathrm{A}^{\mathrm{n}-}+(\mathrm{x} / 2) \mathrm{M}^{2+}(\mathrm{OH})_{2}+ \\
& (\mathrm{x}) \mathrm{H}^{+}
\end{aligned}
$$

and

$$
\begin{aligned}
\Delta_{\mathrm{D}} \mathrm{G}_{\mathrm{m}}^{\mathrm{o}}\{\mathrm{T}, \mathrm{LDH}\}=(1-\mathrm{x}) \Delta_{\mathrm{D}} \mathrm{G}_{\mathrm{m}}^{\mathrm{o}}\left\{\mathrm{T}, \mathrm{M}^{2+}(\mathrm{OH})_{2}\right\}+ \\
\quad(\mathrm{x}) \Delta_{\mathrm{D}} \mathrm{G}_{\mathrm{m}}^{\mathrm{o}}\left\{\mathrm{T}, \mathrm{M}^{3+}(\mathrm{OH})_{3}\right\}-(\mathrm{x})\left[(1 / 2) \Delta_{\mathrm{f}} \mathrm{G}_{\mathrm{m}}^{\mathrm{o}}\left\{\mathrm{T}, \mathrm{M}^{2+}\left(\mathrm{A}^{\mathrm{n}-}\right)_{2 / \mathrm{n}}\right\}-\right. \\
\quad(1 / 2) \Delta_{\mathrm{f}} \mathrm{G}_{\mathrm{m}}^{\mathrm{o}}\left\{\mathrm{T}, \mathrm{M}^{2+}(\mathrm{OH})_{2}\right\}+\Delta_{\mathrm{f}} \mathrm{G}_{\mathrm{m}}^{\mathrm{o}}\left\{\mathrm{T}, \mathrm{OH}^{-}\right\}- \\
\left.\quad(1 / \mathrm{n}) \Delta_{\mathrm{f}} \mathrm{G}_{\mathrm{m}}^{\mathrm{o}}\left\{\mathrm{T}, \mathrm{A}^{\mathrm{n}-}\right\}\right]
\end{aligned}
$$

Again, the term in brackets is the contribution term due to anion difference in the MLCs as defined by Equation 24 and shown in Table 3.

Finally,

$$
\begin{aligned}
& \Delta_{\mathrm{D}} \mathrm{G}_{\mathrm{m}}^{\mathrm{o}}\{\mathrm{T}, \mathrm{LDH}\}=(1-\mathrm{x}) \Delta_{\mathrm{D}} \mathrm{G}_{\mathrm{m}}^{\mathrm{o}}\left\{\mathrm{T}, \mathrm{M}^{2+}(\mathrm{OH})_{2}\right\}+ \\
& (\mathrm{x}) \Delta_{\mathrm{D}} \mathrm{G}_{\mathrm{m}}^{\mathrm{o}}\left\{\mathrm{T}, \mathrm{M}^{3+}(\mathrm{OH})_{3}\right\}-(\mathrm{x}) \Delta_{\mathrm{C}} \mathrm{G}_{\mathrm{m}}^{\mathrm{o}}\left\{\mathrm{T}, \mathrm{M}^{2+} \mathrm{A}\right\}
\end{aligned}
$$

\section{LIMITATIONS OF THE THERMODYNAMIC STUDY}

The validity of the results given in the present work depends greatly on the accuracy of the Gibbs free energy of formation data for the species under consideration. Most of the thermodynamic quantities have been gathered from different sources. All of the standard state properties were determined or estimated by a large diversity of methods and techniques. Therefore, it is expected that some random errors will 
Table 11. Standard molar Gibbs free energy change of the dissolution reaction for some $\mathrm{M}^{3+}$ and $\mathrm{M}^{4+}$ hydroxides at $298.15 \mathrm{~K}$

\begin{tabular}{|c|c|c|c|}
\hline Dissolution Reaction & Equations & $\Delta_{\mathrm{D}} \mathrm{G}_{\mathrm{m}}^{\mathrm{o}}\left\{298.15 \mathrm{~K}, \mathrm{M}^{\mathrm{m}+}(\mathrm{OH})_{\mathrm{n}}\right\}, \mathrm{kJ} \mathrm{mol}^{-1}$ & References \\
\hline $\mathrm{Au}(\mathrm{OH})_{3}(\mathrm{~s})+3 \mathrm{H}^{+}(\mathrm{aq})=\mathrm{Au}^{3+}(\mathrm{aq})+3 \mathrm{H}_{2} \mathrm{O}$ & 20 & 70.8 & $86,87,89$ \\
\hline $\mathrm{Tl}(\mathrm{OH})_{3}(\mathrm{~s})+3 \mathrm{H}^{+}(\mathrm{aq})=\mathrm{Tl}^{3+}(\mathrm{aq})+3 \mathrm{H}_{2} \mathrm{O}$ & $19-20$ & 9.7 & $86,89,92$ \\
\hline $\mathrm{Mn}(\mathrm{OH})_{3}(\mathrm{~s})+3 \mathrm{H}^{+}(\mathrm{aq})=\mathrm{Mn}^{3+}(\mathrm{aq})+3 \mathrm{H}_{2} \mathrm{O}$ & $19-20$ & -2.0 & $86,89,90,92$ \\
\hline $\mathrm{Er}(\mathrm{OH})_{3}(\mathrm{~s})+3 \mathrm{H}^{+}(\mathrm{aq})=\mathrm{Er}^{3+}(\mathrm{aq})+3 \mathrm{H}_{2} \mathrm{O}$ & 20 & -9.9 & $86,87,89$ \\
\hline $\mathrm{Fe}(\mathrm{OH})_{3}(\mathrm{~s})+3 \mathrm{H}^{+}(\mathrm{aq})=\mathrm{Fe}^{3+}(\mathrm{aq})+3 \mathrm{H}_{2} \mathrm{O}$ & $19-20$ & -14.8 & $86,89,92$ \\
\hline $\mathrm{In}(\mathrm{OH})_{3}(\mathrm{~s})+3 \mathrm{H}^{+}(\mathrm{aq})=\mathrm{In}^{3+}(\mathrm{aq})+3 \mathrm{H}_{2} \mathrm{O}$ & $19-20$ & -29.2 & 86,89 \\
\hline $\mathrm{Cr}(\mathrm{OH})_{3}(\mathrm{~s})+3 \mathrm{H}^{+}(\mathrm{aq})=\mathrm{Cr}^{3+}(\mathrm{aq})+3 \mathrm{H}_{2} \mathrm{O}$ & 20 & -33.3 & $86,89,92$ \\
\hline $\mathrm{Ga}(\mathrm{OH})_{3}(\mathrm{~s})+3 \mathrm{H}^{+}(\mathrm{aq})=\mathrm{Ga}^{3+}(\mathrm{aq})+3 \mathrm{H}_{2}^{2} \mathrm{O}$ & $19-20$ & -37.7 & $86,89,92$ \\
\hline $\mathrm{Bi}(\mathrm{OH})_{3}(\mathrm{~s})+3 \mathrm{H}^{+}(\mathrm{aq})=\mathrm{Bi}^{3+}(\mathrm{aq})+3 \mathrm{H}_{2} \mathrm{O}$ & $19-20$ & -46.5 & $86,87,89$ \\
\hline $\mathrm{Al}(\mathrm{OH})_{3}(\mathrm{~s})+3 \mathrm{H}^{+}(\mathrm{aq})=\mathrm{Al}^{3+}(\mathrm{aq})+3 \mathrm{H}_{2}^{2} \mathrm{O}$ & $19-20$ & -46.7 & 86,89 \\
\hline $\mathrm{Gd}(\mathrm{OH})_{3}(\mathrm{~s})+3 \mathrm{H}^{+}(\mathrm{aq})=\mathrm{Gd}^{3+}(\mathrm{aq})+3 \mathrm{H}_{2} \mathrm{O}$ & 20 & -60.2 & $86,87,89$ \\
\hline $\mathrm{Sm}(\mathrm{OH})_{3}(\mathrm{~s})+3 \mathrm{H}^{+}(\mathrm{aq})=\mathrm{Sm}^{3+}(\mathrm{aq})+3 \mathrm{H}_{2} \mathrm{O}$ & 20 & -63.1 & $86,87,89$ \\
\hline $\mathrm{Yb}(\mathrm{OH})_{3}(\mathrm{~s})+3 \mathrm{H}^{+}(\mathrm{aq})=\mathrm{Yb}^{3+}(\mathrm{aq})+3 \mathrm{H}_{2} \mathrm{O}$ & 20 & -69.3 & $86,87,89$ \\
\hline $\mathrm{Sc}(\mathrm{OH})_{3}(\mathrm{~s})+3 \mathrm{H}^{+}(\mathrm{aq})=\mathrm{Sc}^{3+}(\mathrm{aq})+3 \mathrm{H}_{2} \mathrm{O}$ & 20 & -84.8 & 86,89 \\
\hline $\mathrm{Eu}(\mathrm{OH})_{3}(\mathrm{~s})+3 \mathrm{H}^{+}(\mathrm{aq})=\mathrm{Eu}^{3+}(\mathrm{aq})+3 \mathrm{H}_{2} \mathrm{O}$ & $19-20$ & -88.2 & $86,89,92$ \\
\hline $\mathrm{Tb}(\mathrm{OH})_{3}(\mathrm{~s})+3 \mathrm{H}^{+}(\mathrm{aq})=\mathrm{Tb}^{3+}(\mathrm{aq})+3 \mathrm{H}_{2} \mathrm{O}$ & 20 & -89.7 & 87,89 \\
\hline $\mathrm{Dy}(\mathrm{OH})_{3}(\mathrm{~s})+3 \mathrm{H}^{+}(\mathrm{aq})=\mathrm{Dy}^{3+}(\mathrm{aq})+3 \mathrm{H}_{2} \mathrm{O}$ & 20 & -98.6 & 87,89 \\
\hline $\mathrm{Y}(\mathrm{OH})_{3}(\mathrm{~s})+3 \mathrm{H}^{+}(\mathrm{aq})=\mathrm{Y}^{3+}(\mathrm{aq})+3 \mathrm{H}_{2} \mathrm{O}^{2}$ & $19-20$ & -99.9 & $86,89,92$ \\
\hline $\mathrm{Lu}(\mathrm{OH})_{3}(\mathrm{~s})+3 \mathrm{H}^{+}(\mathrm{aq})=\mathrm{Lu}^{3+}(\mathrm{aq})+3 \mathrm{H}_{2} \mathrm{O}$ & 20 & -104.9 & 87,89 \\
\hline $\operatorname{Tm}(\mathrm{OH})_{3}(\mathrm{~s})+3 \mathrm{H}^{+}(\mathrm{aq})=\mathrm{Tm}^{3+}(\mathrm{aq})+3 \mathrm{H}_{2} \mathrm{O}$ & 20 & -105.7 & 87,89 \\
\hline $\mathrm{Nd}(\mathrm{OH})_{3}(\mathrm{~s})+3 \mathrm{H}^{+}(\mathrm{aq})=\mathrm{Nd}^{3+}(\mathrm{aq})+3 \mathrm{H}_{2} \mathrm{O}$ & $19-20$ & -107.8 & $86,89,92$ \\
\hline $\mathrm{La}(\mathrm{OH})_{3}(\mathrm{~s})+3 \mathrm{H}^{+}(\mathrm{aq})=\mathrm{La}^{3+}(\mathrm{aq})+3 \mathrm{H}_{2} \mathrm{O}$ & $19-20$ & -111.6 & $86,89,92$ \\
\hline $\mathrm{Ce}(\mathrm{OH})_{3}(\mathrm{~s})+3 \mathrm{H}^{+}(\mathrm{aq})=\mathrm{Ce}^{3+}(\mathrm{aq})+3 \mathrm{H}_{2} \mathrm{O}$ & $19-20$ & -118.7 & $86,89,92$ \\
\hline $\mathrm{Pu}(\mathrm{OH})_{3}(\mathrm{~s})+3 \mathrm{H}^{+}(\mathrm{aq})=\mathrm{Pu}^{3+}(\mathrm{aq})+3 \mathrm{H}_{2} \mathrm{O}$ & 20 & -127.1 & 87,89 \\
\hline $\operatorname{Pr}(\mathrm{OH})_{3}(\mathrm{~s})+3 \mathrm{H}^{+}(\mathrm{aq})=\mathrm{Pr}^{3+}(\mathrm{aq})+3 \mathrm{H}_{2} \mathrm{O}$ & 20 & -128.3 & 87,89 \\
\hline $\mathrm{Am}(\mathrm{OH})_{4}(\mathrm{~s})+4 \mathrm{H}^{+}(\mathrm{aq})=\mathrm{Am}^{4+}(\mathrm{aq})+4 \mathrm{H}_{2} \mathrm{O}$ & 20 & 42.0 & 87,89 \\
\hline $\mathrm{Zr}(\mathrm{OH})_{4}(\mathrm{~s})+4 \mathrm{H}^{+}(\mathrm{aq})=\mathrm{Zr}^{4+}(\mathrm{aq})+4 \mathrm{H}_{2} \mathrm{O}$ & 20 & 16.2 & 87,89 \\
\hline $\mathrm{Sn}(\mathrm{OH})_{4}(\mathrm{~s})+4 \mathrm{H}^{+}(\mathrm{aq})=\mathrm{Sn}^{4+}(\mathrm{aq})+4 \mathrm{H}_{2} \mathrm{O}$ & 20 & -0.5 & 87,89 \\
\hline $\mathrm{U}(\mathrm{OH})_{4}(\mathrm{~s})+4 \mathrm{H}^{+}(\mathrm{aq})=\mathrm{U}^{4+}(\mathrm{aq})+4 \mathrm{H}_{2} \mathrm{O}$ & 20 & -27.9 & $86,87,89$ \\
\hline $\mathrm{Th}(\mathrm{OH})_{4}(\mathrm{~s})+4 \mathrm{H}^{+}(\mathrm{aq})=\mathrm{Th}^{4+}(\mathrm{aq})+4 \mathrm{H}_{2} \mathrm{O}$ & $19-20$ & -73.6 & $86,89,92$ \\
\hline $\mathrm{Po}(\mathrm{OH})_{4}(\mathrm{~s})+4 \mathrm{H}^{+}(\mathrm{aq})=\mathrm{Po}^{4+}(\mathrm{aq})+4 \mathrm{H}_{2} \mathrm{O}$ & 20 & -112.0 & 87,89 \\
\hline
\end{tabular}

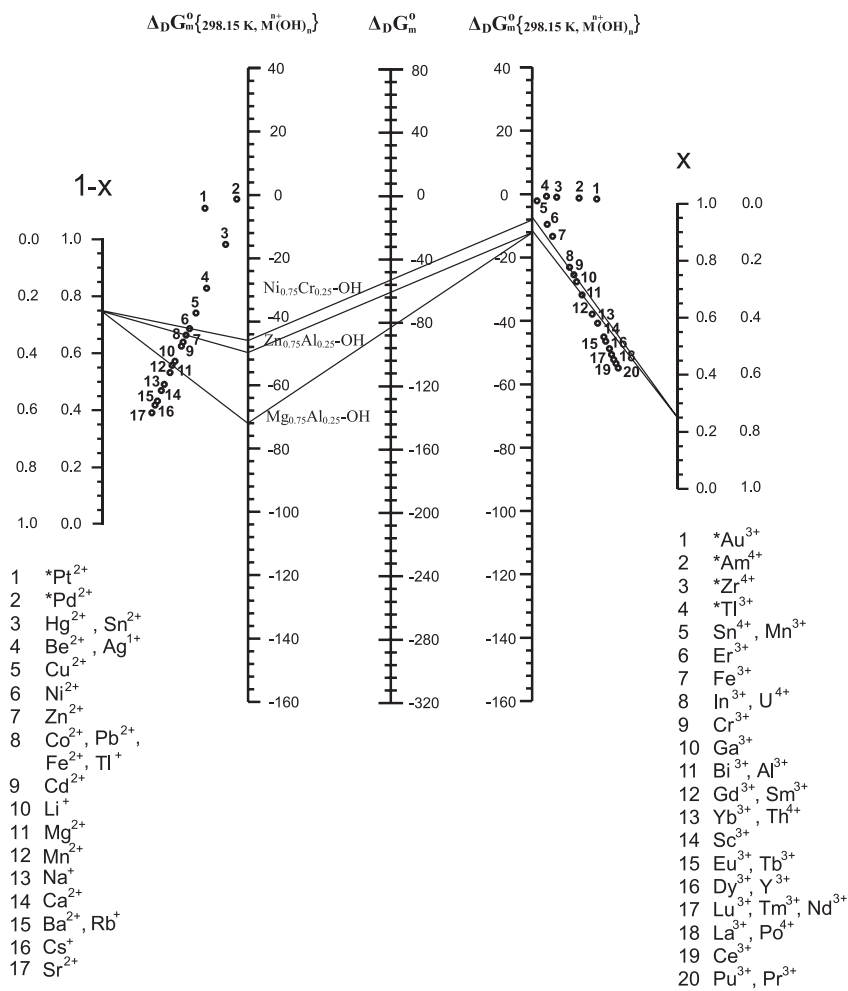

Figure 7. Estimated standard molar Gibbs free energy change of dissolution for MLCs at $298.15 \mathrm{~K}$, in $\mathrm{kJ} \mathrm{mol}^{-1}$ be present due to the variability of the published data. Also, restrictions imposed by the mixture model involving the calculations of standard state properties of formation of LDHs should be taken into account. Although, efforts were made to check for self-consistency of results, this was not always possible due to the lack of data. Although the majority of the thermodynamic quantities (free energies, entropies, enthalpies and heat capacities) were collected from well-known and recognized compilations of thermochemical data, care must be taken when interpreting predicted results.

Moreover, while interpreting results of free energies of reaction, it should not be forgotten that calculations are based on only the species involved in the general reactions. Accordingly, calculations apply only when no other species are present or when these other species do not have a large effect on the overall thermochemical results.

In the present study, information regarding the kinetics of reactions (i.e. reaction rate, activation energy, reaction pathways, etc.) is not provided. For such work, experimental methods are commonly employed.

\section{DISCUSSION}

\section{Synthesis of LDHs by hydrothermal reconstruction methods}

Several studies of LDH synthesis have been carried out, but it was Sato et al. ${ }^{31}$ who first noted a correlation between the hydration reaction of the divalent hydroxide and the easy of reconstitution of the hydrotalcite-like structure for various mixed metal oxides. They 
proposed the following decreasing order for the easy of reconstruction: $\mathrm{Mg}-\mathrm{Al}>\mathrm{Mg}-\mathrm{Fe}>\mathrm{Co}-\mathrm{Al}>\mathrm{Ni}-\mathrm{Al}>\mathrm{Ni}-\mathrm{Fe}$. In light of the data shown in Figure 4, this trend is not surprising because the metal ratio $\mathrm{M}^{3+}$ / $\left(\mathrm{M}^{2+}+\mathrm{M}^{3+}\right)$ of all the starting LDHs was the same, and the standard Gibbs free energies of the hydration of the divalent oxides are quite different. This order is the same as the order predicted by Figure 4 which gives the corresponding free energies, $\Delta_{\mathrm{HR}} \mathrm{G}_{\mathrm{m}}^{\mathrm{o}}\{298.15 \mathrm{~K}, \mathrm{MLC}\}$, of $-21.8 \mathrm{~kJ} \mathrm{~mol}^{-1}<-15.1 \mathrm{~kJ} \mathrm{~mol}^{-1}<-8.6 \mathrm{~kJ} \mathrm{~mol}^{-1}<5.2 \mathrm{~kJ} \mathrm{~mol}^{-1}<11.8$ $\mathrm{kJ} \mathrm{mol}^{-1}$, where the larger the negative value of the free energy of the $\mathrm{H}-\mathrm{R}$ reaction the easier the MLC is formed.

Some other examples ${ }^{28-33,93-94}$ found in the literature, whose synthesis methods are described in detail, are shown in Figure 4. The estimated Gibbs free energy change of reaction agrees qualitatively quite well with the reported observations on the simple preparation of these MLCs. For instance, $\left[\mathrm{Ni}_{1-\mathrm{x}} \mathrm{Al}_{\mathrm{x}}(\mathrm{OH})_{2}\right] \mathrm{OH}_{\mathrm{x}}$ (for $\mathrm{x}=0.25$, $\left.\Delta_{\mathrm{HR}} \mathrm{G}_{\mathrm{m}}^{\mathrm{o}}\{298.15 \mathrm{~K}, \mathrm{MLC}\}=6.3 \mathrm{~kJ} \mathrm{~mol}^{-1}\right)$ is difficult to prepare, and even with high temperature and pressure conditions and prolonged times of reaction, a rather small conversion of the MLC was achieved ${ }^{33}$.

$\left[\mathrm{Mg}_{1-\mathrm{x}} \mathrm{Al}_{\mathrm{x}}(\mathrm{OH})_{2}\right] \mathrm{OH}_{\mathrm{x}}$, known as meixnerite ${ }^{15}$, is so far the most studied MLC, given its relatively smooth formation by reconstruction methods $^{30}$. It should have a fairly negative Gibbs free energy of reaction and Figure 4 supports this result (for $\mathrm{x}=0.25, \Delta_{\mathrm{HR}} \mathrm{G}_{\mathrm{m}}^{\mathrm{o}}\{298.15$ $\left.\mathrm{K}, \mathrm{MLC}\}=-22.6 \mathrm{~kJ} \mathrm{~mol}^{-1}\right)$. It is noteworthy to mention that although the hydration of $\mathrm{Fe}^{3+}$ oxide is not favorable thermodynamically, $\left[\mathrm{Mg}_{1-\mathrm{x}} \mathrm{Fe}_{\mathrm{x}}(\mathrm{OH})_{2}\right] \mathrm{OH}_{\mathrm{x}}$ can be prepared successfully thanks to the contribution of the $\mathrm{Mg}^{2+}$ free energy of hydration to the total free energy of the LDH reconstruction ${ }^{93}$.

Data of hydration of some $\mathrm{M}^{+}$and $\mathrm{M}^{4+}$ oxides have also been included in Figure 4 since they have been introduced in the LDH layers and even synthesized by hydrothermal methods ${ }^{94}$. This is also the case for $\left[\mathrm{Li}_{1-\mathrm{x}} \mathrm{Al}_{\mathrm{x}}(\mathrm{OH})_{2}\right][\mathrm{OH}]_{2 \mathrm{x}-1}{ }^{95}$, which is shown in Figure 4 to have a favorable Gibbs free energy of reaction (for $\mathrm{x}=0.66$, $\Delta_{\mathrm{HR}} \mathrm{G}_{\mathrm{m}}^{\mathrm{o}}\{298.15 \mathrm{~K}, \mathrm{MLC}\}=-19.9 \mathrm{~kJ} \mathrm{~mol}^{-1}$ ).

$\mathrm{LDH}$ reconstruction in the presence of non-hydroxyl anions has been carried out even more often than the preparation of MLCs. This might be due to the increasing interest of this method for environmental applications ${ }^{58-61,64}$ (i.e. anion sequestering) and for the intercalation of organic and inorganic anions in the LDH layers ${ }^{96-}$ 99. Sato et al. ${ }^{29}$ reported that the degree of adsorption of $\mathrm{Mg}_{1-\mathrm{x}} \mathrm{Al}_{\mathrm{x}}$ oxides of divalent anions such as $\mathrm{CrO}_{4}^{2-}, \mathrm{HPO}_{4}^{2-}, \mathrm{HVO}_{4}^{2-}, \mathrm{SiO}_{3}^{2-}$, $\mathrm{HGaO}_{3}^{2-}$ and $\mathrm{SO}_{4}^{2-}$ was higher than that of the monovalent anions $\mathrm{Cl}^{-}$ and $\mathrm{MnO}_{4}^{-}$. This conforms with the results in Table 3 and shows that $\Delta_{\mathrm{C}} \mathrm{G}_{\mathrm{m}}^{\mathrm{o}}\{298.15 \mathrm{~K}, \mathrm{MgA}\}$ of $\mathrm{SO}_{4}^{2-}$ is more favorable thermodynamically to the formation of $\mathrm{LDH}$ than that of $\mathrm{Cl}^{-}\left(\Delta_{\mathrm{C}} \mathrm{G}_{\mathrm{m}}^{\mathrm{o}}\left\{298.15 \mathrm{~K}, \mathrm{MgSO}_{4}\right\}=\right.$ $\left.49.4 \mathrm{~kJ} \mathrm{~mol}^{-1}<\Delta_{\mathrm{C}} \mathrm{G}_{\mathrm{m}}^{\mathrm{o}}\{298.15 \mathrm{~K}, \mathrm{MgCl}\}=94.9 \mathrm{~kJ} \mathrm{~mol}^{-1}\right)$. Châtelet $e t$ al. ${ }^{100}$ also found similar results.

Table 3 can be also used to predict anion selectivities for the reconstruction of LDHs for a specific divalent-trivalent metal-mixed oxide. However, care should be taken when using these data. For example, Parker et al. ${ }^{63}$ found anion absorption selectivities for $\mathrm{Mg}$ Al mixed oxides of: $\mathrm{SO}_{4}{ }^{2-}>\mathrm{F}^{-}>\mathrm{Cl}^{-}>\mathrm{NO}_{3}{ }^{-}$and the anion selectivities predicted by Table 3 are: $\mathrm{F}^{-}>\mathrm{SO}_{4}{ }^{2-}>\mathrm{NO}_{3}{ }^{-}>\mathrm{Cl}^{-}$. Although, these predictions contradict the experimental results, these differences may be explained on the basis of the $\mathrm{CO}_{3}{ }^{2-}$ impurities present in the aqueous solution ${ }^{63}$ and the oxide adsorption capacities ${ }^{100}$, factors not taken into account in the thermodynamic calculations.

\section{Synthesis of LDHs by the coprecipitation (CP) method}

As seen in Table 8, there are many possible combinations of metal salts and anions that may be used in the CP method. Therefore, only the most common combinations will be discussed. Since Feitknecht's work $^{4}$, hydrotalcite, $\left[\mathrm{Mg}_{1-\mathrm{x}} \mathrm{Al}(\mathrm{OH})_{2}\right]\left(\mathrm{A}^{\mathrm{n}-}\right)_{\mathrm{x} / \mathrm{n}}$, has been the most studied
LDH. According to Cavani et al. ${ }^{9}$ this may be because hydrotalcite is simple and relatively inexpensive to prepare in the laboratory. Examination of the terms contributing to the total Gibbs free energy in Tables 4 and 5 for $\mathrm{Mg}$ and $\mathrm{Al}$, respectively, reveals that $\mathrm{Mg}$ and $\mathrm{Al}$ salts are the ones with the highest negative contribution value. Hence, the ease of synthesis of hydrotalcite can be understood readily.

There has been a clear preference to use metal chlorides as starting materials for $\mathrm{CP}$ synthesis. However, metal nitrates have also been employed $^{6,9-10}$. Although different kinds of anions have been introduced in LDHs, carbonate anions appear to be the most common'. Carbonate anions have also appeared as an impurity in the final LDH, even if a decarbonated synthesis media was used ${ }^{6}$. Detailed calculations of standard Gibbs free energy of the synthesis of some common LDHs are given in Table 9. From these results, some interesting points arise: 1) In all cases, hydrotalcites have relatively high negative Gibbs free energies of reaction; 2) Higher negative Gibbs free energies of $\mathrm{CP}$ reaction are obtained when metal chlorides are used as precursors; 3) Carbonate anions are thermodynamically favored in the LDH interlamellar region due to their small $\Delta_{C} G_{m}^{o}\left\{298.15 \mathrm{~K}, \mathrm{M}^{2+} \mathrm{A}\right\}$ values compared to nitrate and chloride anions. These findings agree with the experimental observations.

\section{Solubility of LDHs}

In spite of the importance of the solubility of LDHs, related thermodynamic data in the literature are rather scarce ${ }^{78}$. Sato et al. ${ }^{31}$ calculated the solubilitites of some LDHs (Mg-Al, Mg-Fe, Ni-Al, $\mathrm{Ni}-\mathrm{Fe}$ and $\mathrm{Co}-\mathrm{Al})$. Using $\mathrm{K}_{\mathrm{sp}}{ }$ values $\left(\mathrm{K}_{\mathrm{sp}}{ }_{\mathrm{sp}}=\left[\mathrm{M}^{2+}\right]\left[\mathrm{OH}^{-}\right]\right)$they concluded that the solubility of LDHs decreased appreciably when $\mathrm{Mg}^{2+}$ was replaced with $\mathrm{Ni}^{2+}$ and $\mathrm{Co}^{2+}$ and that an appreciable difference was not noted when $\mathrm{Al}^{3+}$ was replaced with $\mathrm{Fe}^{3+}$. A similar conclusion can be drawn from the results in Figure 7. Comparing the $\mathrm{M}^{2+}$ contributions to the total free energy of dissolution, a decreasing degree of solubility gives the order $\mathrm{Mg}^{2+}>\mathrm{Co}^{2+}>\mathrm{Ni}^{2+}$. This order is based on the fact that the larger the negative value of the free energy of the dissolution reaction the more soluble the LDH. Similarly, it can be seen that Co-Al LDHs will have a lower negative free energy of dissolution than the corresponding cobalt hydroxide, as shown by Thompson et al. ${ }^{101}$. Ranges of $\mathrm{pH}$ in which some metal hydroxides and LDHs may exist have been reported ${ }^{10,102}$. Some examples of $\mathrm{pH}$ ranges for LDHs are shown in Table 12.

Looking at the results in Figure 7 for LDH combinations in Table 12, a reasonable correlation between the lower $\mathrm{pH}$ limit and the free energy change of dissolution is observed. This result is not surprising since LDHs dissolve in acidic media. The standard Gibbs free energies of dissolution have been tabulated in the last column of Table 12 . The $\mathrm{Zn}-\mathrm{Cr}$ compound deviates the most from the correlation, and this may be due to a kinetic effect because of a special stability of the compound. Likewise, $\mathrm{Zn}-\mathrm{Al}, \mathrm{Zn}-\mathrm{Cr}$ and $\mathrm{Ni}-\mathrm{Al} \mathrm{LDH}$ s have been used in the synthesis of intercalated catalysts at low $\mathrm{pH}$ because of their greater resistance to dissolution compared to $\mathrm{Mg}-\mathrm{Al} \mathrm{LDH}^{54,103}$. The influence of interlamellar anions on the solubility of LDHs has been little studied. Allada et al. ${ }^{78}$ reported that nitrate and sulfate anions could increase the solubility of hydrotalcite relative to the carbonate hydrotalcite. The same trend is obtained from $\Delta_{\mathrm{C}} \mathrm{G}_{\mathrm{m}}^{\mathrm{o}}\{298.15 \mathrm{~K}, \mathrm{MgA}\}$ data for $\mathrm{NO}_{3}{ }_{3}^{-}, \mathrm{SO}_{4}{ }^{2-}$ and $\mathrm{CO}_{3}{ }^{2-}$ in Table $3\left(\Delta_{\mathrm{C}} \mathrm{G}_{\mathrm{m}}^{\mathrm{o}}\left\{298.15 \mathrm{~K}, \mathrm{MgNO}_{3}\right\}\right.$ $=76.7 \mathrm{~kJ} \mathrm{~mol}^{-1}>\Delta_{\mathrm{C}} \mathrm{G}_{\mathrm{m}}^{\mathrm{o}}\left\{298.15 \mathrm{~K}, \mathrm{MgSO}_{4}\right\}=49.4 \mathrm{~kJ} \mathrm{~mol}^{-1}>$ $\left.\Delta_{\mathrm{C}} \mathrm{G}_{\mathrm{m}}^{\mathrm{o}}\left\{298.15 \mathrm{~K}, \mathrm{MgNO}_{3}\right\}=17.8 \mathrm{~kJ} \mathrm{~mol}^{-1}\right)$.

\section{New LDH materials}

Numerous LDH materials have been reported so far; however, the number of compositions and possible combinations of metals 
Table 12. $\mathrm{pH}$ ranges at which some LDHs may exist $\mathrm{t}^{10}$

\begin{tabular}{|c|c|c|c|}
\hline \multirow[b]{2}{*}{ LDH } & \multicolumn{2}{|c|}{ pH Range } & \multirow[t]{2}{*}{$\Delta_{\mathrm{D}} \mathrm{G}_{\mathrm{m}}^{\mathrm{o}}\{298.15 \mathrm{~K}, \mathrm{LDH}\}, \mathrm{kJ} \mathrm{mol}^{-1}$} \\
\hline & Low Limit & High Limit & \\
\hline$\left[\mathrm{Zn}_{1-\mathrm{x}} \mathrm{Cr}_{\mathrm{x}}(\mathrm{OH})_{2}\right]\left(\mathrm{Cl}^{-}\right)_{\mathrm{x} / \mathrm{n}}$ & 4.5 & 10.0 & -74.1 \\
\hline$\left[\mathrm{Ni}_{1-\mathrm{x}} \mathrm{Al}_{\mathrm{x}}(\mathrm{OH})_{2}\right]\left(\mathrm{CO}_{3}^{2-}\right)_{x / \mathrm{n}}$ & 5.0 & 10.0 & -62.4 \\
\hline$\left[\mathrm{Ni}_{1-\mathrm{x}} \mathrm{Cr}_{\mathrm{x}}(\mathrm{OH})_{2}\right]\left(\mathrm{Cl}^{-}\right)_{\mathrm{x} / \mathrm{n}}$ & 5.5 & 11.5 & -69.9 \\
\hline$\left[\mathrm{Zn}_{1-\mathrm{x}} \mathrm{Al}_{\mathrm{x}}(\mathrm{OH})_{2}\right]\left(\mathrm{Cl}^{-}\right)_{\mathrm{x} / \mathrm{n}}$ & 6.0 & 10.0 & -77.4 \\
\hline$\left[\mathrm{Co}_{1-\mathrm{x}} \mathrm{Fe}_{\mathrm{x}}(\mathrm{OH})_{2}\right]\left(\mathrm{Cl}^{-}\right)_{\mathrm{x} / \mathrm{n}}$ & 7.5 & 10.5 & -73.0 \\
\hline$\left[\mathrm{Mg}_{1-\mathrm{x}} \mathrm{Al}_{\mathrm{x}}(\mathrm{OH})_{2}\right]\left(\mathrm{Cl}^{-}\right)_{\mathrm{x} / \mathrm{n}}$ & 8.0 & 10.5 & -107.4 \\
\hline
\end{tabular}

present in the layers and anions in the interlamellar region is almost unlimited. As seen from Figure 1, there are still lots of possibilities to design novel LDH compounds with almost any metal in the periodic table and whose properties have not been yet fully investigated.

The synthesis of LDHs with new divalent and trivalent metals, different from the classically used, represents an open and current field of research. For instance, very few articles referencing LDH materials with lanthanoid and actinoid elements in the LDH layers have been reported $^{40,104}$. Some monovalent and tetravalent metals have been also used in the synthesis of LDHs. Some examples are $\mathrm{Li}^{+95}, \mathrm{Na}^{+41}, \mathrm{Ti}^{4+51}$ and $\mathrm{Zr}^{4+45,105}$. Other $\mathrm{M}^{4+}$ metals with proper ionic radius can be anticipated (i.e. $\mathrm{Am}^{4+}, \mathrm{V}^{4+}, \mathrm{Os}^{4+}$ among others). A different situation occurs with most of the single valent $\mathbf{M}^{+}$. From Figure 1, it can be seen that the ionic radii of most of the $\mathrm{M}^{+}$metals have great differences with the ionic radius of $\mathrm{Mg}^{2+}$ (i.e. $\mathrm{K}^{+}, \mathrm{Rb}^{+}, \mathrm{Cs}^{+}, \mathrm{Fr}^{+}, \mathrm{Au}^{+}, \mathrm{Tl}^{+}$and $\mathrm{Ag}^{+}$ among others). This fact might explain in part why they have not been introduced so far in the layers of LDH compounds. The same problem is observed with divalent metals such as $\mathrm{Sr}^{2+}$ and $\mathrm{Ba}^{2+}$, although they have been claimed in some patents ${ }^{46-49}$.

Using the results of Figures 1 and 4, it can be easily predicted that some combination of metals are highly favored thermodynamically to form LDHs. For example, from the family of $\mathrm{LDH}$ compounds: $\mathrm{Li}^{+}-\mathrm{M}^{3+} \mathrm{LDHs}$, only the $\mathrm{Li}^{+}-\mathrm{Al}^{3+} \mathrm{LDH}$ has been reported ${ }^{95}$ and attempts to synthesize $\mathrm{Li}^{+}-\mathrm{Cr}^{3+}, \mathrm{Li}^{+}-\mathrm{Fe}^{3+}, \mathrm{Li}^{+}-\mathrm{Ga}^{3+}$ and $\mathrm{Li}^{+}-\mathrm{In}^{3+} \mathrm{LDHs}$ presumably by coprecipitation methods have failed ${ }^{106}$. In this particular case, hydrothermal methods using metal oxides of $\mathrm{Li}^{+}$and $\mathrm{Fe}^{3+}, \mathrm{Ga}^{3+}$ or $\mathrm{In}^{3+}$ as starting materials are thermodynamically favored for the formation of LDHs, as predicted by Figure 4, and could be a route to the successful synthesis of these materials.

The free energy anion contribution term, $\Delta_{\mathrm{C}} \mathrm{G}_{\mathrm{m}}^{\mathrm{o}}\left\{298.15 \mathrm{~K}, \mathrm{M}^{2+} \mathrm{A}\right\}$, given in Table 3 and used in Equation 25 might be used to predict anion selectivities for the reconstruction of LDH compounds. For example, $\mathrm{Fe}^{2+}-\mathrm{M}^{3+}$ metal mixed oxides might show monovalent anion selectivities of $\mathrm{OH}^{-}>\mathrm{NO}_{3}^{-}>\mathrm{F}^{-}>\mathrm{Cl}^{-}>\mathrm{Br}^{-} \approx \mathrm{I}^{-}$and divalent anion selectivities of $\mathrm{CO}_{3}{ }^{2-}>\mathrm{SO}_{4}{ }^{2-}$. A similar order of stability in acidic solutions is expected for $\mathrm{Fe}^{2+}-\mathrm{M}^{3+} \mathrm{LDHs}$ as calculated by Equation 48. Figure 7 can be used to predict LDH compounds resistant to dissolution; for example, the introduction of metals such as $\mathrm{Pt}^{2+}, \mathrm{Pd}^{2+}$, $\mathrm{Sn}^{2+}, \mathrm{Be}^{2+}, \mathrm{Au}^{3+}, \mathrm{Am}^{4+}, \mathrm{Zr}^{4+}, \mathrm{Tl}^{3+}$ and $\mathrm{Mn}^{3+}$ among others should improve the stability of LDH materials in acid media.

\section{CONCLUSIONS}

From the present thermodynamic review of the synthesis of LDH materials, good qualitative agreement between the estimated standard molar Gibbs free energies and the published experimental data for the synthesis and solubility of LDHs is found. Even though these results are encouraging, care should be taken when interpreting the data, especially when trying to do a quantitative analysis. For instance, carbonate anions are supposed to be highly favorable thermodynamically in the synthesis of LDHs. However, from the results in Table
3, carbonate anions are in some cases slightly unfavorable to the formation of LDHs when compared to hydroxyl anions as in $\mathrm{Mg}^{2+}$ $\mathrm{M}^{3+}$ LDHs. Allada et al. ${ }^{78}$ estimated the errors associated with the predictions of thermodynamic data by the mixture model to be within 5 to $10 \mathrm{~kJ} \mathrm{~mol}^{-1}$. Therefore, differences found in the anion contributions from Table 3 between carbonate and hydroxyl anions fall within the error range given by the model. Also, the aqueous chemistry of the ions involved in the general reactions may be different and influence the final thermodynamic result.

In general terms, the results reported in the present work represent a valuable tool to assist in the synthesis of LDHs by H-R and CP methods. This study also helps to predict the solubility of LDH compounds, opening up the possibility of creating a great number of new, interesting materials.

\section{ACKOWLEDGEMENTS}

We acknowledge financial support for this work by the Director, Division of Chemical and Thermal Systems of the National Science Foundation under grant CTS-0321979, and from Universidad Industrial de Santander and COLCIENCIAS, in the frame of the project "Synthesis, characterization and testing of biomimetic catalysts for selective oxidation", code 1102-05665-95.

\section{REFERENCES}

1. Allman, R.; Neues Jahrb. Mineral., Monatsh. 1969, 12, 544.

2. Allman, R.; Chimia 1970, 24, 99.

3. Hochstetter, C.; J. Prakt. Chem. 1842, 27, 375.

4. Feitknecht, W.; Helv. Chim. Acta 1942, 25, 131.

5. Miyata, S.; Kumura, T.; Chem. Lett. 1973, 843.

6. Miyata, S.; Clays Clay Miner. 1975, 23, 369.

7. Reichle, W. T.; Chemtech 1986, 16, 58.

8. Reichle, W. T.; Solid State Ionics 1986, 22, 135.

9. Cavani, F.; Trifiro, F.; Vaccari, A.; Catal. Today 1991, 11, 173.

10. de Roy, A.; Forano, C.; El Malki, K.; Besse, J. P. In Synthesis of Micrporous Materials; Ocelli, M. L.; Robson, M. E., eds.; Van Nostrand Reinhold: New York, 1992, vol. II, p. 108.

11. Trifiro, F.; Vaccari, A. In Comprehensive Supramolecular Chemistry; Atwood, J. L.; Davies, J. E. D.; MacNicol, D. D.; Vogtle, F.; Lehn, J. M.; Alberti, G.; Bein, T., eds.; Pergamon: Oxford, 1996, vol. 7, p. 251.

12. Sels, B. F.; de Vos, D. E.; Jacobs, P. A.; Catal. Rev. Sci. Eng. 2001, 43, 443.

13. Crepaldi, E. L.; Valim, J. B.; Quim. Nova 1998, 21, 300.

14. Rives, V.; Layered Double hydroxides: Present and Future, Nova Science Publishers, Inc: New York, 2001.

15. Koritnig, S; Süsse, P.; Tschermaks Min. Petr. Mitt. 1975, 22, 79.

16. Lopez, T.; Bosch, P.; Ramos, E.; Gomez, R.; Novaro, O.; Acosta, D.; Figueras, F.; Langmuir 1996, 12, 189.

17. Delmas, C.; Borthomieu, Y.; J. Solid State Chem. 1993, 104, 345.

18. Roy, D. M.; Roy, R.; Osborn, E. F.; Am. J. Sci. 1953, 251, 337.

19. Mascolo, G.; Marino, O.; Mineral. Mag. 1980, 43, 619.

20. Mascolo, G.; Appl. Clay Sci. 1995, 10, 21.

21. Martin, E. S.; Pearson, A.; U.S. Patent 5,514,361 1996.

22. Indira, L.; Dixit, M.; Kamath, P. V.; J. Power Sources 1994, 52, 93.

23. Miyata, S.; Clays Clay Miner. 1983, 31, 305. 
24. Bish, D. L.; Bull. Mineral. 1980, 103, 170.

25. Drezdzon, M. A.; Inorg. Chem. 1988, 27, 4628.

26. Crepaldi, E. L.; Pavan, P. C.; Valim, J. B.; J. Mater. Chem. 2000, 10, 1337.

27. Leroux, F.; Adachi-Pagano, M.; Intissar, M.; Chauvière, S.; Forano, C.; Besse, J. P.; J. Mater. Chem. 2001, 11, 105.

28. Miyata, S.; Clays Clay Miner. 1980, 28, 50.

29. Sato, T.; Wakabayashi, T.; Shimada, M.; Ind. Eng. Chem. Prod. Res. Dev. 1986, $25,89$.

30. Sato, T.; Kato, K.; Endo, T.; Shimada, M.; React. Solids 1986, 2, 253.

31. Sato, T.; Fujita, H.; Endo, T.; Shimada, M.; Tsunashima, A.; React. Solids 1988, 5, 219.

32. Kooli, F.; Depège, C.; Ennaqad, A.; de Roy, A.; Besse, J. P.; Clays Clay Miner. 1997, 45, 92

33. Prinetto, F.; Tichit, D.; Teissier, R.; Coq, B.; Catal. Today 2000, 55, 103.

34. Easley, M. A.; U.S. Patent 5,645,810 1997.

35. Feitknecht, W.; Gerber, M.; Helv. Chim. Acta 1942, 25, 106.

36. Vichi, F. M.; Alves, O. L.; J. Mater. Chem. 1997, 7, 1631.

37. Rousselot, I.; Taviot-Gueho, C.; Besse, J. P.; Int. J. Inorg. Mater. 1999, 1, 165 .

38. Aramendia, M. A.; Borau, V.; Jimenez, C.; Marinas, J. M.; Luque, J.M.; Ruiz, J.R. Urbano, F.; J. Mater. Lett. 2000, 43, 118.

39. Basile, F.; Fornasari, G.; Gazzano, M.; Vaccari, A.; Appl. Clay. Sci. 2000, $16,185$.

40. Perez-Ramirez, J.; Kapteijn, F.; Moulijn, J.; Catal. Lett. 1999, 60, 133.

41. Wen, Z. Y.; Lin, Z. X.; Chen, K. G.; J. Mater. Sci. Lett. 1996, 15, 105.

42. Velu, S.; Susuki, K.; Kapoor, M. P.; Tomura, S.; Ohashi, F.; Osaki, T.; Chem. Mater. 2000, 12, 719.

43. Labajos, F. M.; Sastre, M. D.; Trujillano, R.; Rives, V.; J. Mater. Chem. 1999, 9, 1033

44. Fernandez, J. M.; Barriga, C.; Ulibarri, M. A.; Labajos, F. M.; Rives, V.; Chem. Mater. 1997, 9, 312.

45. Tichit, D.; Das, N.; Coq, B.; Duran, R.; Chem. Mater. 2002, 14, 1530.

46. Miyata, S.; Jpn. Kokai Tokkyo Koho JP 610002071986.

47. Miyata, S.; U.S. Patent 4,710,551 1987.

48. Miyata, S.; Kumura, T.; Shimada, M.; U.S. Patent 3,879,523 1970.

49. Miyata, S.; Kumura, T.; Shimada, M.; U.S. Patent 3,796,792 1970.

50. Okusako, A.; Miyazaki, S.; Jpn. Kokai Tokkyo Koho JP 20003172702000.

51. Taylor, R. M.; Clay Miner. 1984, 19, 591.

52. Spamer, A.; Nkosi, B.; PCT Int. Appl. WO 01478412001.

53. Shannon, R. D.; Acta Crystallogr., Sect. A: Found. Crystallogr. 1976, 32, 751.

54. Rives, V.; Ulibarri, M. A.; Coord. Chem. Rev. 1999, 181, 61.

55. Bejoy, N.; Reson. J. Sci. Ed. 2001, 6, 57.

56. Peterson, C. L.; Perry, D. L.; Masood, H.; Lin, H.; White, J. L.; Hem, S. L.; Fritsch, C.; Haeusler, F.; Pharm. Res. 1993, 10, 998.

57. Shin, H. S.; Kin, M. J.; Nam, S. Y.; Moon, H. C.; Water Sci. Technol. 1996, $34,161$.

58. Reardon, E. J.; Della Valle, S.; Environ. Sci. Technol. 1997, 31, 1218.

59. Goswamee, R. L.; Sengupta, P.; Bhattacharyyn, K. G.; Dutta, D. K.; Appl. Clay Sci. 1998, 13, 21.

60. Lehmann, M.; Zouboulis, A. I.; Matis, K. A.; Chemosphere 1999, 39, 881.

61. Camino, G.; Maffezzoli, A.; Braglia, M.; De Lazzaro, M.; Zammarano, M.; Polym. Degrad. Stab. 2001, 74, 457.

62. van der Ven, L; van Gemert, M. L. M.; Batenburg, L. F.; Keern, J. J.; Gielgens, L. H.; Koster, T. P. M.; Fischer, H. R.; Appl. Clay Sci. 2000, 17, 25.

63. Parker, L. M.; Milestone, N. B.; Newman, R. H.; Ind. Eng. Chem. Res. 1995, 34, 1196.

64. Ulibarri, M. A.; Hermosín, M. C. In ref. 14, p. 251

65. Ambrogi, V.; Fardella, G.; Grandolini, G.; Perioli, L.; Int. J. Pharm. 2001, $220,23$.

66. Constantino, U.; Nocchetti, M. In ref. 14, p. 383.

67. Gardner, E.; Huntoon, K. M.; Pinnavaia, T. J.; Adv. Mater. 2001, 13, 1263.

68. Pernice, P.; Marino, O.; Mascolo, G.; Thermochim. Acta 1988, 133, 87.

69. de Roy, A.; Besse, J. P.; Solid State Ionics 1991, 46, 95.

70. Itaya, K.; Chang, H. C.; Uchida, I.; Inorg. Chem. 1987, 26, 624.

71. Ballarin, B.; Seeber, R.; Tonelli, D.; Vaccari, A.; J. Electroanal. Chem. 1999, $463,123$.
72. Morigi, M.; Scavetta, E.; Berrettoni, M.; Giorgetti, M.; Tonelli, D.; Anal. Chim. Acta 2001, 439, 265.

73. Leggat, R. B.; Zhang, W.; Buchheit, R. G.; Taylor, S. R.; Corrosion Houston, TX, U.S. 2002, 58, 322.

74. Buchheit, R. G.; Mamidipally, S. B.; Schmutz, P.; Guan, H.; Corrosion Houston, TX, U.S. 2002, 58, 3 .

75. Buchheit, R. G.; J. Appl. Electrochem. 1998, 28, 503.

76. Boclair, J. W.; Ph.D. Thesis, University of North Texas, Texas, USA, 1998

77. Pitsch, S.; Krishnamurthy, R.; Arrhnius, G.; Helv. Chim. Acta 2000, 83, 2398.

78. Allada, R. K.; Navrotsky, A.; Berbeco, H. T.; Casey, W. H.; Science 2002, 296, 721.

79. Hansen, H. C. B.; Borggaard, O. K.; Sørensen, J.; Geochim. Cosmochim. Acta 1994, 58, 2599.

80. Israëli, Y.; Taviot-Guého, C. Besse, J.P.; Morel, J.P.; Morel-Desrosiers, N.; J. Chem. Soc., Dalton Trans. 2000, 791.

81. Bravo-Suárez, J. J.; Páez-Mozo, E. A.; Oyama, S. T.; Quim. Nova 2004, 27,574

82. Hussein, M. Z. B.; Zainal, Z.; Yahaya, A.; Foo, D. W. V.; J. Controlled Release 2002, 82, 417.

83. Khan, A. I.; O'Hare, D.; J. Mater. Chem. 2002, 12, 3191.

84. Barner, H. E.; Sheuerman, R. V.; Handbook of Thermochemical Data for Compounds and Aqueous Species, John Wiley \& Sons: New York, 1978.

85. Binnewies, M.; Milke, E.; Thermochemical Data of Elements and Compounds, Wiley-VCH: New York, 1999.

86. Naumov, G. B.; Ryzhenko, B. N.; Khodakovsky, I. L.; Handbook of Thermodynamic Data, U.S. Geological Survey: Washington, D.C., 1974.

87. Karapet'yants, M. Kh.; Karapet'yants, M. L.; Thermodynamic Constants of Inorganic and Organic Compounds, Ann Arbor-Humphrey Science Publishers: Ann Arbor, MI, 1970.

88. Knacke, O.; Kubaschewski, O.; Hesselmann, K.; Thermochemical Properties of Inorganic Substances, Springer -Verlag: Berlin, 1991.

89. Perry, H. R.; Green, D. W.; Maloney, J. O.; Perry's Chemical Engineers' Handbook, McGraw-Hill: New York, 1997.

90. Latimer, W. M.; Pitzer, K. S.; Smith, W. V.; J. Am. Chem. Soc. 1938, 60, 1829.

91. Price, G. J.; Thermodynamics of Chemical Processes, Oxford University Press: Oxford, New York, 1998.

92. The heat capacity, $\mathrm{C}_{\mathrm{pm}}^{\mathrm{o}}$, of the metal hydroxide has been estimated in 83.7 $\mathrm{J} \mathrm{mol}^{-1} \mathrm{~K}^{-1}$, which is approximately the average value among different published data of $\mathrm{C}_{\mathrm{p}, \mathrm{m}}^{\mathrm{o}}$ for several $\mathrm{M}^{+}, \mathrm{M}^{2+}, \mathrm{M}^{3+}$ and $\mathrm{M}^{4+}$ hydroxides. A deviation of about $40 \mathrm{~J} \mathrm{~mol}^{-1} \mathrm{~K}^{-1}$ from the average $\mathrm{C}_{\mathrm{p}, \mathrm{m}}^{\mathrm{o}}$ value only introduces a maximum change in the total free energy of reaction of up to $2 \mathrm{~kJ} \mathrm{~mol}^{-1}$ and would be noticeable at high temperatures.

93. Hibino, T.; Tsunashima, A.; J. Mater. Sci. Lett. 2000, 19, 1403.

94. Nemudry, A. P.; Isupov, V. P.; Kotsupalo, N. P.; Boldyrev, V. V.; React. Solids 1986, 1, 221.

95. Serna, C. J.; Rendon, J. L.; Iglesias, J. E.; Clays Clay Miner. 1982, 30, 180

96. Chibwe, K.; Jones, W.; J. Chem Soc., Chem. Commun. 1989, 926.

97. Chibwe, K.; Jones, W.; Chem. Mater. 1989, 1, 489

98. Dimotakis, E. D.; Pinnavaia, T. J.; Inorg. Chem. 1990, 29, 2393.

99. Narita, E.; Kaviratna, P.; Pinnavaia, T. J.; Chem. Lett. 1991, 805.

100. Châtelel, L.; Bottero, J. Y.; Yvon, J.; Bouchelaghem, A.; Colloids Surf., A 1996, 111,167

101. Thompson, H. A.; Parks, G. A.; Brown, G. E.; Clays Clay Miner. 1999, 47,425

102. Blesa, M. A.; Morando, P. J.; Regazzoni, A. E.; Chemical Dissolution of Metal Oxides, CRC Press: Boca Raton, FL, 1994.

103. Kwon, T.; Tsigdinos, G. A.; Pinnavaia, T. J.; J. Am. Chem. Soc. 1988, 110 , 3653 .

104. Li, L.; Hui, J.; Liu, X.; Zhang, Y.; Xu, R.; Gaodeng Xuexiao Huaxue Xuebao 1993, 14, 1048; Chem. Abstr. 1994, 120, 297618.

105. Velu, S.; Sabde, D. P.; Shah, N.; Sivasanker, S.; Chem. Mater. 1998, 10, 3451.

106. Rajamathi, M.; Thomas, G. S.; Kamath, P. V.; Proc. Indian Acad. Sci. Chem. Sci. 2001, 113, 671. 